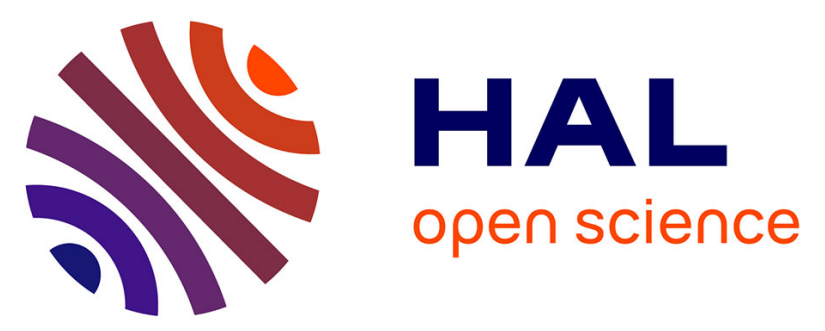

\title{
Invasions of Host-Associated Microbiome Networks
}

\author{
C. L. Murall, J. L. Abbate, M. Puelma Touzel, E. Allen-Vercoe, Samuel
}

Alizon, Remy Froissart, K. Mccann

\section{To cite this version:}

C. L. Murall, J. L. Abbate, M. Puelma Touzel, E. Allen-Vercoe, Samuel Alizon, et al.. Invasions of Host-Associated Microbiome Networks. Research, BT - Advances in Ecological. Networks of Invasion: Empirical Evidence and Case Studies, 57, Academic Press, 2017, Advances in Ecological Research, 10.1016/bs.aecr.2016.11.002 . hal-01567903

\section{HAL Id: hal-01567903 \\ https://hal.science/hal-01567903}

Submitted on 24 Jul 2017

HAL is a multi-disciplinary open access archive for the deposit and dissemination of scientific research documents, whether they are published or not. The documents may come from teaching and research institutions in France or abroad, or from public or private research centers.
L'archive ouverte pluridisciplinaire HAL, est destinée au dépôt et à la diffusion de documents scientifiques de niveau recherche, publiés ou non, émanant des établissements d'enseignement et de recherche français ou étrangers, des laboratoires publics ou privés.

\section{다)(1) $(5$}

Distributed under a Creative Commons Attribution - NonCommerciall 4.0 International 


\title{
Invasions of Host-Associated Microbiome Networks
}

Chapter for the volume "Networks of Invasion" in the book series "Advances in Ecological Research"

Participating authors:

Carmen Lia Murall ${ }^{1}$, Jessica L. Abbate ${ }^{2}$, Maximilian Puelma Touzel ${ }^{3}$, Emma Allen-Vercoe ${ }^{4}$, Samuel Alizon ${ }^{1}$, Remy Froissart ${ }^{1}, K_{\text {Kevin McCann }}^{5}$

Affiliations:

1. Laboratoire MiVEGEC, CNRS, IRD, Montpellier, France

2. Laboratoire MiVEGEC, IRD, Montpellier, France

3. Ecole Normale Supérieure de Paris, Département de Physique, Paris, France

4. Department of Molecular and Cellular Biology, University of Guelph, Guelph, Canada

5. Department of Integrative Biology, University of Guelph, Guelph, Canada

\begin{abstract}
The study of biological invasions of ecological systems has much to offer research on withinhost systems, particularly for understanding infections and developing therapies using biological agents. Thanks to the ground-work established in other fields, such as community ecology and evolutionary biology, and to modern methods of measurement and quantification, the study of microbiomes has quickly become a field at the forefront of modern systems biology. Investigations of host-associated microbiomes (e.g., for studying human health) are often centered on measuring and explaining the structure, functions and stability of these communities. This momentum promises to rapidly advance our understanding of ecological networks and their stability, resilience and resistance to invasions. However, intrinsic properties of host-associated microbiomes that differ from those of free-living systems present challenges to the development of a within-host invasion ecology framework. The elucidation of principles underlying the invasibility of within-host networks will ultimately help in the development of medical applications and help shape our understanding of human health and disease.
\end{abstract}

Keywords: within-host communities, polymicrobial interactions, infections, colonisation, community stability, microbial community dynamics, microbiome therapies, immunity-microbiota interactions 


\section{Table of Contents}

\section{Introduction}

2. Ecological and evolutionary concepts applied to microbiome networks: in the face of invaders

2.1 Invasions and ecological stability

2.2 Insights from empirical invasion ecology

2.3 Competitive interactions

2.4 Evolution and mutualisms

Table 1. Network properties of microbiome and ecological communities and their implications for invasibility

3. Quantifying invasions of the microbiome: data, modelling and theory

3.1 Quantitative formulation of a microbiome

3.2 Measuring the microbiome

3.3 Inferring the interaction network

3.4 Theoretical microbiome invasion dynamics

\section{Unique features of within-host communities}

4.1 The immune system

4.1.1 Host barriers and the innate response

4.1.2 Rapidly evolving predation

4.1.3 Immunity controls community composition and dynamics

4.2 Resident species actively prevent invasions

4.3 Fatally virulent invaders

Box 1: Does relatedness help or hurt a species ability to invade?

\section{Experimental tests of microbiome invasibility}

\section{Types of invaders}

6.1 Microbial (single and polymicrobial invasions)

6.2 Bacteriophages

Box 2: Unique features of other kinds of invaders

\section{Actively acquired invasions}

7.1 Bacteriophage therapies

7.2 Probiotics and food-borne invaders

7.3 Other therapies

7.4 A note on non-living perturbations

\section{Conclusions and Perspective}




\section{Introduction}

Never before has there been such widespread appreciation that ecology happens not just around us but inside us. The ongoing discovery of the complexity of the web of microorganisms in and on our bodies has led to a rapidly emerging, systems-level picture of our within-host (WH) ecosystem. Due to the recent advancements of '-omics' techniques and increasingly sensitive methods to probe immunity and host cells, it is now possible to study the ecology and evolution happening inside the human body at an unprecedented level of resolution and quantification. Arising from this is a challenge similar to the one ecologists face for free-living ecosystems: how can we harness community (i.e., network) level knowledge in order to predict the consequences of disturbances? In particular, what makes a $\mathrm{WH}$ community susceptible to or protected against invasion?

There is a wide range of biological invasions that can enter our bodies, from various kinds of micro- to macro-organisms (e.g., bacteria, viruses, worms, etc.) to human-created invaders (e.g., live vaccines, probiotics, bacteriophage therapies, etc.). Consequently, knowledge about biological invasions of the $\mathrm{WH}$ ecosystem is scattered across the scientific literature. The introduction of a non-native biological agent into a $\mathrm{WH}$ system is usually referred to as an infection. A species introduced into a WH system is considered a successful within-host invader when it is able to establish and maintain a viable population in or on the host. While most research is interested in infections that cause disease, i.e. pathogenic invaders, successful within-host invasion does not necessarily harm or kill the host. An invasive species in a WH system may be beneficial, i.e. it may share a symbiotic relationship with the host. Similarly, in free-living systems an invading species is considered to be invasive if it has a disruptive or negative impact on the resident community; while frequent, this is not always the case. Here, we will consider invasions generally and thus include discussions about biological agents invading $\mathrm{WH}$ ecosystems regardless of medical implications to the host or negative impacts on the resident community.

The extent of the interdisciplinarity of WH studies is a challenge. Many insights, concepts and discoveries are found in very distant fields with often little communication with one another (e.g., microbiology, immunology, various clinical fields, community ecology, evolutionary biology, and invasion biology where insights are often found in the plant or conservation literature). Also, the discovery of the members of WH communities has created a picture of an inter-kingdom network, replete with bacteria, viruses, fungi and archaea (Horz 2015; Huffnagle and Noverr 2013; Lecuit and Eloit 2013; Nobbs and Jenkinson 2015). We believe that ecological and evolutionary theory can be a unifying framework from which patterns and principles can emerge.

There have been several attempts at synthesizing ecological methods and concepts to aid researchers studying the microbiome (e.g., reviews by Costello et al. 2012; Ley et al. 2006; Pepper and Rosenfeld 2012). Here, our focus is more targeted. We wish to illustrate how insights from the ecology and evolution of biological invasions can help us understand the 
massive and ever-growing knowledge about host-associated microbiomes. We use 'microbiome' to mean the collection of microorganisms in and on its host and this also encompasses the set of genes from these microorganisms, their products and environment (Boon et al. 2014). These, then, are host-associated 'biomes'. We also discuss when these ecosystems exhibit novel features distinct from free-living ecosystems. Given the immense variety of invading species that can enter host-associated ecosystems, we will focus our examples mainly on invasions of bacterial communities by other bacteria and bacteriophages. We complement this selection with a non-exhaustive set of other examples chosen either for their importance in the development of the field or because they exhibit unique features worthy of mention. We end by highlighting the various invasions and perturbations that humans actively induce (e.g., probiotics or antibiotics) and finally conclude with a perspective of future directions for those interested in applying a community ecology and network approach.

\section{Ecological and evolutionary concepts applied to microbiome networks: in the face of invaders}

\subsection{Invasion and ecological stability}

Community ecology has a long, rich history of studying the stability of ecological systems (Ives and Carpenter 2007; May 1972; McCann 2000; Namba 2015). The application of this knowledge, theory and methods should play a central role in the understanding of the human within-host ecosystem. Indeed, humans can be seen as an ecosystem with many interacting species, perturbations, spatial structure, environmental gradients, and successional patterns. In addition, studying invasions of the microbiome can help elucidate the underlying mechanisms that stabilise and destabilise these ecological systems.

The meaning of a 'stable community' can differ depending on the definition of stability or what metric is used (for more on stability's multi-dimensionality and other details see (Donohue et al. 2013; Ives and Carpenter 2007; McCann 2012). Two important concepts, rooted in dynamical systems theory, arise. A steady state is one where, in the absence of perturbations, the behaviour of the system remains constant over time. When a steady state is stable, the natural 
dynamics of the system will take its state to this stable state, i.e. to an attractor, which can have fixed, oscillating, or even chaotic values. The return time refers to how quickly a system can return to a stable steady state that it is perturbed away from (this is also called resilience in ecology). Changing parameters can alter the stability of steady states, for example by making them suddenly unstable, whereupon the natural dynamics takes the state away to some other steady state that is stable. Most theoretical studies of ecological stability focus on stable states while empirical studies use temporal (or spatial) variability of abundance or biomass to measure stability, where communities with wildly fluctuating populations are considered less stable (Donohue et al. 2016).

The theoretical literature on invasion and its implication on stability are twofold. One, there exists a literature deriving largely from Charles Elton (1958) that addresses invasion resistance, which is measured as a change in magnitude of abundance and biomass after an invasion, and it is related to persistence, where a community that is difficult to invade is considered persistent. For a summary of the various hypotheses to explain successful invasions, including Elton's biotic resistance hypothesis, (see Inderjit et al. 2005; in particular their Table 1), and Section 2.2 below for discussion of classic empirical studies. A second, far less developed area, considers the impacts of invasions on the stability of the pre-existing whole system. One metric used for this is robustness, which is the number of extinctions after an invasion. Charles Elton with his enormous historical influence weighed in on both of these aspects of invasion and stability by broadly arguing that more diverse ecosystems tend to be more resistant to invasion and less affected (Elton 1958).

Since Elton (1958), there has been a strong push for understanding the role of diversity on invasion resistance, usually through intra- or interspecific competition. Case (Case 1990; Case and Bolger 1991) and others (Drake 1990; Law and Morton 1996; Levine and Antonio 1999), employed communities matrices capable of producing complex communities (May 1973) to show that, on average, more diverse communities were more resistant to invasion. In an excellent synthetic single trophic level contribution, Tilman (1999) argued that much of diversity stability theory agreed with Elton's premise that increased diversity tends to decrease invasion success. Specifically, Tilman (1999) argued that increasing diversity has the tendency, on average, to reduce equilibrium resource levels, $R^{*}$. Since lower resource levels inhibit successful invasion, it remains that diverse communities should be more resistant (Tilman 1999). While interesting, this perspective is largely considered from a negative interaction perspective, and others (e.g., Lawlor 1979; Levine and Antonio 1999) have pointed out that species interactions that include facilitative effects can alter this result in such a way that increased diversity can actually decrease invasion resistance.

In the past 20 years both the general ecological literature on diversity-stability (e.g., McCann 2000) and the invasion resistance literature discussed above (e.g., Levine and Antonio 1999) have developed these arguments to find that diversity, in and of itself, is likely not the greatest contributor to stability in the face of invasion. The diversity-stability literature, for example, has argued that the structure and positioning of interaction strengths can dramatically increase stability relative to the random community approaches (McCann 2011). One finding is that weak 
interactions, in low or high diversity trophic webs, can act to mute out the destabilising impacts of strong, highly suppressive or oscillatory, interactions (Gellner and Mccann 2016). Indeed, in the largest analysis of empirical food webs to date, non-random structures, such as a high frequency of weak interactions and their topology, were found to stabilise complex food webs (Jacquet et al. 2016). From an invasion perspective, these results strongly suggest that invasive species with rapid growth potential and low mortality (e.g. the invader has no resident predator in the newly invaded community) are extremely likely to not only invade, but reach high densities, and act as potent destabilisers of even diverse communities (see single trophic level ideas that are similar by (Tilman 1999). Thus, both demographic attributes (high growth potential) and a species position within the food web contrive to yield massive destabilising impacts on the resident food web.

\subsection{Insights from empirical invasion ecology}

Empirical investigations of invasions of ecological systems have given ecologists insights into how ecological communities assemble and evolve over time (see Amsellem et al. 2017; Jackson et al. 2017; Medoc et al. 2017 in this issue). While there are several classic lessons that invasions have taught us about the ecology and evolution of communities (e.g. rapid geographical range changes are possible, speciation can be sympatric, etc.), more recent studies have pushed this body of knowledge further (e.g. communities are rarely saturated with species, adaptive genetic change can be occur quickly, etc.; see Sax et al. 2007 for a review). It can be argued that in these early years of microbiome studies, invasions have also played an important role in helping probe these networks. We will review some examples of these microbiome studies in later sections. Invasions of WH communities are implicated in a number of human diseases, particularly those induced by infectious pathogens, as well as in determining the success of prophylactic and therapeutic biological control agents. Therefore, invasion ecology of the $\mathrm{WH}$ ecosystem will continue to not only be central to teaching us how these systems work but is also crucial for applied research. Here we present some insights from invasions of free-living systems that might be of use to those studying $\mathrm{WH}$ systems.

The field of invasion ecology is focused primarily on what conditions determine either success at or susceptibility to invasion by a novel species into a resident community. From the perspective of the invader, this action is broken down into a set of successive processes that must be executed: (1) dispersal out of the native environment, (2) colonisation of a suitable new environment, (3) successful establishment in the new environment, and (4) spread across the novel landscape (Theoharides and Dukes 2007). Experimental studies have explored the traits that favour or hinder these processes, and how each step contributes to the success and impact of the invasion. No fewer than twenty-nine different hypotheses have been posited in the literature to explain either what determines a successful alien invader, or what factors might render a resident community vulnerable to invasion (recently reviewed in (Jeschke 2014). Some of the most pervasive and pertinent to invasions of the microbiome, discussed or alluded to later in this chapter, include the biotic resistance hypothesis (as mentioned above), the adaptive evolution hypothesis (the ability of a species to respond to selective pressures, see (Keller 
and Taylor 2008 for a synthesis), and the invasional facilitation hypothesis or 'invasional meltdown', which posits that successive invasions happen through time due to facilitative interactions between invaders (Inderjit et al. 2005; Simberloff and Holle 1999).

Empirical investigations into some of these hypotheses have found a number of biological properties that contribute to invasion success. Here, we describe a few well-supported properties, such as dispersal, adaptation, and phylogenetic relatedness.

Dispersal out of the native environment (invasion step 1) and colonisation into the new environment (invasion step 2) are processes that can be likened to epidemiological transmission of infectious agents (regardless of pathogenicity). If there is a low probability of success for each agent's propagule to disperse (make contact) and establish (cause infection) in a new host, then this can be offset by increasing the number of propagules leaving the original infected host (if the agent is pathogenic then this is equivalent to increasing its virulence) or, otherwise, by increasing the amount of contact events with the prospective new host (Alizon and Michalakis 2015; Gilligan and Van Den Bosch 2008). Indeed, in free-living communities, species with higher fecundity were more often a priori classified as invasive (Burns et al. 2013). The intuitive finding that species with life-history strategies that divert more energy to reproduction were more successful invaders than those that put more energy into survival (e.g., having higher overwintering success) is consistent with the growing evidence that suggests propagule pressure (i.e. the frequency and abundance of newly arriving, hopeful invaders) is one of the most important indicators of establishment in a new range (Lockwood et al. 2005). High propagule pressure can help an invading species achieve threshold densities of individuals necessary for population viability (e.g., Allee effects; Hufbauer et al. 2013). This feature of invasibility is likely to be shared with WH systems, as many pathogens are thought to only be successful after achieving a threshold of infection pressure (Simberloff 2009), minimum infectious dose (Yezli and Otter 2011), or infection spillover events between species that often precede full-blown host shifts (Lloyd-Smith et al. 2009). Hartfield and Alizon (2014) laid out a mechanistic process by which epidemiological feedbacks may allow for this to happen, and this theory could be of use to help understand the determinants of successful establishment for an invader, particularly if the invader has arrived from a very different environment (e.g., not from direct transmission between hosts). However, too much propagule pressure can actually limit the success of an invasion, because colonisers with advantageous mutations in the new environment may be out-competed (in terms of opportunity for establishment) due to their rarity among other propagules that are more adapted, on average, to the home range (and not the new range). This is termed gene swamping and has been shown to hinder the ability to maintain novel adaptations to the new environment in small peripheral populations (Antonovics 1976; Antonovics 1968). While an organism that can reproduce without a partner is not limited by finding a suitable mate in the new range, clonality, self-fertilization, or even inbreeding within large homogeneous founder events can limit the probability of successful invasion (e.g., Hufbauer et al. 2013).

On the other hand, prior adaptation, i.e. selection in the native habitat before invasion that produces traits conferring an evolutionary advantage in the introduced habitat (also sometimes 
termed 'pre-adaptation'; see (Hufbauer et al. 2012) for a detailed description), can be important for biological invasions. For example, a comparative study among wildflower species in the family Caryophyllaceae showed that traits that improved home range size were tightly correlated with successful invasion in the new range (Jenkins and Keller 2011). In cases such as these, one would expect different success rates by an invader coming from a completely different environment (e.g., abiotic/environmental transmission) vs. from inside another host (e.g., direct contact or ingestion) or displacement within the same host (e.g., auto-inoculation). Along these lines, Tilman (1999) also pointed out that invaders from an entirely separate ecosystem may often evolve under different conditions giving them traits (e.g., low edibility) that produce a suite of particularly successful advantages in another differently evolved ecosystem.

The determination of successful invasion is likely to involve the interaction between several factors. Gallien et al. (2014) used community vegetation data from the French Alps to illustrate the insight offered by invasive species distribution models that incorporate biotic interactions with environmental and dispersal components. Whether through shared resources or escape from predators, invasion steps 3 (establishment) and 4 (spread), in particular, must be viewed in the context of the resident species in the new environment. Rather than intrinsic traits or a general rule, the advantageous characteristics of an invader depend on the functional and phylogenetic similarities shared with taxa in the resident community, and these relationships can change depending on the scale (Carboni et al. 2016) or environmental conditions (Burns 2006) under which they are measured. However, there is a debate concerning the positive or negative impacts of relatedness on an invasion, as related resident taxa may either prime the environment to facilitate similar taxa (or simply indicate a permissive environment for similarly adaptive traits, termed the pre-adaptation hypothesis) or serve as exclusive competitors already occupying the shared 'niche' making it harder to invade, i.e. Darwin's naturalisation hypothesis (this is discussed in greater detail by Pantel et al. 2017 in this issue). In the context of the $\mathrm{WH}$ microbiome, where the sheer number and diversity of species interactions can be overwhelming, consideration of functional trait similarities may prove more informative than seemingly ever-changing taxonomic classifications of microbiota (Martiny et al. 2015). This is important to establish, because a recent meta-analysis on the generality of relatedness effects on invasion success and impact showed that broad generalities appear to exist across a wide range of plant and animal studies (Ma et al. 2016). Furthermore, experimental results from a longitudinal study of plant communities showed that the invader can quickly drive the native relative to extinction ( $\mathrm{Li}$ et al. 2015), thus increasing the chances that this is missed by simple association surveys. This may be of particular importance for disease studies, in which the microbiota is typically assessed in a snapshot fashion after the invasion has occurred.

It is also helpful to view the biological properties of the resident community as dynamic rather than static. Thus, it matters when an invader enters along the evolutionary or ecological trajectory of the resident community. This invokes yet another major axis of fundamental ecology, ecological succession, or the progression of biological community assembly through time. In forest ecology, from which this literature was born, the pervasive idea is that there is a relatively predictable progression from a virgin environment devoid of life to a stable community of species best adapted to the habitat. Theory, and decades of empirical support from free-living 
species, posits that while the most successful of initial colonisers to a new community will be those with $\boldsymbol{r}$-selected lifestyles (investing in a large number of fast-growing and wide-spreading offspring), those with more K-selected lifestyles (investing in fewer, larger, slower-growing offspring) that arrive later tend to be better competitors in the recently colonised environment (Duyck et al. 2004; Duyck et al. 2006; Facon et al. 2005; Facon et al. 2008). The speed and timing of this succession can therefore influence what taxa, and traits, are those considered successful for invasion. In the case of food webs, trophic level and generalism play such a role, i.e. islands are first colonised by producers, then primary consumers, and so forth (see Massol et al. 2017 in this issue). This is particularly applicable to how our bodies are colonised after birth. We are born essentially germ-free, and the development from infant to childhood to adulthood is a long complex series of colonisation events (Koenig et al. 2011). While the concept also helps generate expectations for re-colonisation of the microflora after perturbation (such as antibiotic exposure), we will discuss in later sections how succession has been important in experimental studies of the microbiome, which are often conducted on axenic in vivo or in vitro systems for tractability.

Finally, a classic insight from invasion ecology is that, counter-intuitively, resident species are not always optimally adapted to their environment (Sax et al. 2007). The potential that invaders are better adapted to the host environment than resident species is underappreciated in microbiome studies (Cho and Blaser 2012). It is tempting to view a resident WH species (commensal or mutualistic) as being highly adapted to their environment and host (e.g., Ley et al. 2006), and the expectation that symbiotic bacteria must be highly adapted to very specialized host niches (Danielsson et al. 2011) is found in the microbiome literature. However, the fact that some invaders can successfully invade and outperform local species in some functions means that the local strategy may not be the true optimal strategy, but rather is the best that the local species can do given its own evolutionary history or constraints. Like in free-living systems, resident bacterial taxa will have some traits that are more highly specialised than others. Similarly, community composition is often not due to ancient co-evolutionary histories with their host but rather communities can be formed uniquely by species interactions (via fitting and sorting), and as a consequence, communities dominated by exotic species can form and persist (Sax et al. 2007).

\subsection{Competitive interactions and invasions}

Competitive species-species interactions are often found to destabilise ecological community dynamics (Ives and Carpenter 2007) but yet competitive interactions in light of invasions can help to maintain stability of the resident system. Indeed, in WH systems, competitive interactions of resident species can help prevent invasions. For instance, gut microbiota species in leeches have been shown to prevent invasions by production of antibiotics (Graf et al. 2006; Tasiemski et al. 2015). Also, experimental evolution studies in a nematode model showed how a resident bacteria rapidly evolved increased antimicrobial superoxide production as a defense against Staphylococcus aureus infections (King et al. 2016). In a human example, lactic acid 
producing bacterial species lower the $\mathrm{pH}$ of the vaginal microbiome environment, which subsequently prevents the establishment of not only bacteria but also viruses (Danielsson et al. 2011). Importantly, resident bacterial species produce compounds (e.g., bacteriocins) to directly inhibit growth of invading bacteria thus using interference competition as a mechanism to prevent invasion (Dethlefsen et al. 2007). In a recent study, a cell-to-cell contact-dependent antagonism (where strains of Bacteroidetes inject toxic effectors into their neighbours) was described in detail (Wexler et al. 2016). This phylum is very common in the human microbiome and thus, by demonstrating that this antagonistic behaviour affects the overall community composition and dynamics, they suggest that this interaction could be significantly shaping human microbiome communities. Locally, this antagonism allows these strains to limit their direct competition for resources but still benefit from diffusible compounds from other bacteria nearby (though not directly touching).

Interestingly, competition and not cooperative interactions appear to be more common in cultured communities (Foster and Bell 2012). Similarly a study, that fit mathematical (ecologically based) models to time series data from the guts of mice, found that competitive interactions were most common, and in fact, parasitic/predatory and amensalistic interactions were also more common than cooperative interactions (Marino et al. 2014). Further, they found that intra-phylum interactions between all Firmicutes were competitive. These experimental results appear to help support a recent theoretical result that showed that microbial communities with more competitive interactions than cooperative interactions are more stable, with the caveat that too many competitive interactions can also have a destabilising effect (Coyte et al. 2015).

In free-living systems, invasions by competitors rarely cause complete extinction of endemic species (Sax et al. 2007), thus, suggesting that using a competitive strategy alone does not necessarily make invaders effective at disrupting the resident community. However, the same cannot be said for invading predators and pathogens which are more successful strategies for invasion (Sax et al. 2007), thus implying that these negative interactions can be more effective at destabilising the system. Insights such as these are particularly important for studies that wish to eradicate an unwanted resistant species using invaders as biological control (such as bacteriophage therapies) because using pathogens or possibly combining predators and competitors could be potentially more effective than solely introducing competitors.

There are other factors that matter as to whether competitive interactions are stabilising or destabilising, namely, the strength of the interactions and the topological context. For example, competitive interactions that form transitive (hierarchical) or intransitive (cyclic) networks have been shown to affect not only stability (e.g., networks of competitive interactions are stabilising; Allesina and Levine 2011) but also promote or hinder diversity. For instance, Kerr et al. (2002) showed that $E$. coli strains coexist when interactions are spatially explicit, thus echoing theoretical work on the coexistence of species in structured communities (Hofbauer and Schreiber 2010; Mouquet and Loreau 2002). Indeed, experiments (in vivo or in vitro) of microbiome invasions, particularly those that combine them with ecological mathematical modelling (e.g., Stein et al. 2013; Marino et al. 2014; Wexler et al. 2016), could help further 
probe the properties of the WH networks that make competitive interactions either effective or ineffective at preventing invasions.

\subsection{Evolution of cooperation and mutualism}

\subsubsection{Interactions within microbial species}

Altruistic strategies consist in conferring a fitness benefit to another individual at a personal cost. By definition, these are at the mercy of defecting strategies that do not pay the helping cost (but accept the benefits). There are two ways to explain how altruistic strategies can out-compete cheating strategies. First, individuals may have means to identify altruists, which is known as kin discrimination (Hamilton 1964). One caveat, however, is that a defecting individual may end up bearing the same discrimination tag as altruists. Interestingly, cooperation can endure if one allows for enough different tag values and for mutation in this trait (Jansen and van Baalen 2006); an idea which has been applied to siderophore production (Lee et al. 2012). The second process, also introduced by Hamilton (1964), through which altruists can out-compete defectors is known as kin selection and depends on the "viscosity" of the environment. If the environment is spatially structured and if individuals are born locally and interact locally, then on average altruists should interact more with fellow, related altruists. As a group, altruists are then by definition fitter than defectors such that clusters of altruists can out-compete clusters of defectors. Much of the microbiota is found on epithelial surfaces and therefore dwell in highly structured environments (e.g. the gut or the skin), which means kin selection effects should be strong: even if a parasitic strain manages to create a small cluster, it is likely to be outcompeted by clusters of cooperators that are more productive.

\subsubsection{Interactions between microbial species}

Positive reciprocal interactions between microbiota members are often cited as important protectors of $\mathrm{WH}$ invasions. Yet, the mechanisms for this are less clear. For example, a recent theoretical study has challenged this idea by showing that mutualistic interactions, in fact, weaken communities by introducing feedbacks that lessen the stability of the network (Coyte et al. 2015). That mutualisms themselves can be destabilising is something already known in general community ecology theory (Namba 2015) and so considering other features that are linked to mutualisms, such as nestedness or evolution need to be considered. For instance, another theoretical study (not specific to the microbiome) shows that the evolutionary process itself is stabilising, and that, for low diversity networks, evolution of mutualistic interactions are also stabilising, but this changes for larger networks (Loeuille 2010). However, Rohr et al. (2014) argued that mutualistic systems have architectures that allow them to widen the parameter region where species coexistence is possible and thus are more persistent than often appreciated. In addition, adding a third mutualist to a pair of cooperators can increase the cost of cooperation (Harcombe et al. 2016), thus suggesting that increasing the number of competitors may weaken a network. Overall, then, more work is needed to understand the role 
microbe-microbe cooperative interactions have on stability and resilience of the whole community.

Earlier modelling has identified three factors that may lead to mutualistic interactions, namely (i) high benefit to cost ratio, (ii) high within-species relatedness and (iii) high between-species fidelity (Foster and Wenseleers 2006). One challenge to model microbe evolution is that of horizontal gene transfer. Indeed, if the trait of interest, i.e. the one used to define the level of cooperation/mutualism, is carried on a mobile genetic element, the classic relatedness definition, which is based on identity by descent, becomes obsolete and requires adjustments (Smith 2001; Rankin et al. 2011).

Horizontal gene transfer can play a striking role in the adaptation of microbial communities to fluctuating host environments. Interestingly, recent evidence suggests that the invasion of parasitic bacteria is itself a fuel for horizontal gene transfer (Stecher et al. 2013). Indeed, dysbiosis (a microbiome community that is pathogenic to the host, and is often the consequence of a successful invasion) is characterised by the growth of bacteria that were either rare or absent before the perturbation. The proliferation of these invaders represents an opportunity for the resident microbiota to acquire new genes via horizontal transfer. There is, of course, a risk that this blooming of rare bacteria destabilises the whole microbial community. However, the benefits might also outweigh the costs because of a tendency for the loss of genes that are redundant in a community. Morris et al. (2012) name it the Black queen effect and their idea is that at the genotype level, there is a selective pressure to get rid of nonessential genes that are present in other species in the community. The name of the process originates from the fact that, at some point, one genotype bears the last copy of the gene and cannot get rid of it without destabilising the whole community. This whole black queen effect is likely to destabilise the community (since gene redundancy has a stabilising effect at the community level), which is why dysbiosis could in the end prove somehow useful at the community level. Note that this is also a clear illustration of how, perhaps contrarily to free-living ecosystems, evolutionary and ecological processes regularly overlap in WH communities.

\subsubsection{Interaction between host and microbes}

It is commonly said that hosts have coevolved with their microbiome to have a strong mutualistic relationship. However, specific human-microbe coevolution examples are difficult to find, possibly because most of the evidence relies on co-phylogenetics (i.e., comparing the phylogeny of the hosts to that of its bacteria) and because it is extremely difficult to disentangle human evolution from phenotypic plasticity or development (but see Moeller et al. 2016 for an example on hominid-microbe coevolution). For instance, the gut microbiota is strongly affected by the host's diet and changes at the host population level (e.g., due to migration or external changes in the environment) do not require human evolution. In fact, it is easy to have a fixed view of interactions. As is also found in free-living systems, the mutualistic or parasitic nature of a resident microbe can very well depend on the environment and thus can shift without evolutionary changes. For instance, $G B$ virus $C$ is usually a commensal virus in humans but it becomes beneficial if there is a co-infection (i.e., simultaneous infection) with HIV (Bhattarai and 
Stapleton 2012; Tillmann et al. 2001; Xiang et al. 2001). Envisaging human-microbiome interactions as "dangerous liaisons" (i.e., allowing for dynamic partnerships and also for environmental feedbacks; van Baalen and Jansen 2001), is perhaps key to understanding the ecology and evolution of this relationship. Epidemiological modelling can also help understand interactions between hosts and microbial species. It has been known for a long time that infections can have pleotropic effects on their host (Michalakis et al. 1992). The challenge, however, is that this effect on the host likely depends on the within-host parasite community and few epidemiological models allow for co-infections by more than two parasite species (but see Sofonea et al. 2015) and even fewer allow for host evolution. Incorporating diverse microbial communities into a detailed epidemiological setting remains an open challenge.

\section{Quantifying invasions of the microbiome: data, modelling and theory}

Progress in our understanding of invasions into microbiomes will likely require parallel developments in data acquisition, model inference techniques, and theoretical investigations. In this section, we begin with a discussion of the formulation of a microbiome that is amenable to quantitative analysis. We then summarize ecologically motivated approaches from each of the measurement, inference and mathematical theory of microbiomes relevant to invasion analyses.

\subsection{Quantitative formulation of a microbiome}

Despite the additional challenge posed by definitions of the species concept for microbial organisms, an ecological community as an interacting network of species populations remains a useful concept for microbiome research. Attempts to quantify this network face many of the same challenges found in quantifying of those of free-living communities. The microbiome exhibits a diversity that parallels that of free-living systems. We are finding many more phyla than expected and there is a great deal of inter-host variability (e.g., not more, and likely less, than $30 \%$ of fecal bacteria species are shared across individuals; Faith et al. 2013). Thus, determining and then understanding even a specific microbiome network of even a single region of a single individual's whole within-host ecosystem appears daunting (e.g., see Nobbs and Jenkinson 2015) which reviews the diverse players of the inter-kingdom network of the oral ecosystem). The many players include bacteria, host cells (as the physical environment and as resources), bacteriophages, yeast, fungi, viruses and, not the least, immunity cells and compounds. Nevertheless, we are rapidly discovering and characterizing what fungi (i.e., the mycobiota (Huffnagle and Noverr 2013; Underhill and Iliev 2014), viruses (i.e., the virome; 
Delwart 2013; Foxman and Iwasaki 2011; Minot et al. 2011; Minot et al. 2013; Wylie et al. 2013), and archaea are present in human bodies (Lurie-Weinberger and Gophna 2015). Much of this progress comes from the attractive advantages that microbiomes offer to ecology over those of free-living systems: shorter generation times, larger samples, and high-throughput sequencing methods, which together open up the possibility of measuring abundances of a significant fraction of microbiome species over timescales at which relevant community dynamics occurs.

Given the diversity of the microbiome, the scope of study is often narrowed with the research question onto only a subset of players. This carries the risk of neglecting important contributors. At the very least, however, the currently studied sub-networks provide a practical starting point from which to incorporate more players. As the field matures and the methods achieve more depth with less measurement issues, we are likely to access significant fractions of most regional microbiome networks.

Just as with free-living ecosystems, directed links between nodes in a microbiome network signify significant asymmetric energy transfer, termed interaction strength. Defining and measuring interaction strength is important (Berlow et al. 2004; McCann 2000; O'Gorman et al. 2010), especially given the array of interactions WH ecological systems engage in. Capturing multiple forms of species interactions is also of interest to ecologists and recent work has proposed 'inclusive networks' which capture multiple kinds of interactions more mechanistically (e.g., feeding and non-feeding; Kéfi et al. 2012).

At a deeper resolution, and in contrast to the taxonomy of free-living ecosystems, current bacterial species definitions are often constructed by applying user-defined (and thus subjective) sequence-similarity thresholds to cluster the data into so-called operational taxonomic units (OTUs). However, despite the high genetic variability within bacterial species, in part due to their ability to pass genes between individuals, this clustering is in principle informative because it is typically performed on regions of 16S ribosomal RNA (rRNA), which contain highly variable regions that are nonetheless highly conserved within a bacterial species. Longer sequencing reads now lower the sensitivity of the clustering on the choice of this subjective threshold (Franzén et al. 2015), and attempts at more robust threshold-based and non-threshold-based definitions are being proposed (Rossi-Tamisier et al. 2015; Puillandre et al. 2012).

As studies in some other complex biological networks have suggested (Marder and Taylor 2011), such a detailed dissection of the system might obscure rather than clarify the principles at work. Indeed, a healthy microbiome network might be functionally redundant or degenerate regarding the set of functions that it provides for the host. A redundant community is one in which multiple species play similar roles. These communities are in principle robust up to perturbations that knock out all but one of the members within one such functional group. A similarity of function can also exist between two species in two instances of the same community across a pair of hosts in which case the community is degenerate. By particular life histories/contingency etc., different host individuals may then have different bacterial actors 
performing in a functionally equivalent community. This degeneracy would lead to highly variable species-level measurements across individuals, hiding the underlying functional organization. A current direction of research regarding a microbiome definition is then the determination of functional classes of microbiome members. Members of the same group would serve the same function (Shafquat et al. 2014; Boon et al. 2014), and it is hoped that this grouping would better capture the community's topology and dynamics. One popular option is to group taxa based on their metabolic activities, e.g. grouping by catabolisers of polysaccharides or proteins or by creating respiratory guilds (for a review of such models see Song et al. 2014). A particular grouping, Clusters of Orthologous Groups (COGs), classes proteins into gross functions of the cell such as energy production and conversion, cell cycle control and mitosis, and replication and repair (Tatusov et al. 2003). While seemingly natural, such characterizations have so far been subjective and the need to make them less so remains. In particular, many species fulfill the same basic functions with similar pathways, and so distinguishing species via these functions is not likely meaningful. At the other extreme, many species exhibit unique functions that make comparisons ill-defined. Choosing the appropriate 'depth' in the degree of variation of functions across the community and knowing which functions are active and important for community dynamics, both present subtlety and require careful consideration. The singular focus of these genetic and molecular approaches on genes and their expression make them fundamentally different from traditional ecological approaches, such as trophic-level and guild methods of network construction. Attempts to carry over techniques or make comparisons to these traditional methods may be fruitful in some cases and senseless in others.

Also important is the question of how resolved the temporal measurements need to be in order to capture the relevant $\mathrm{WH}$ dynamics of the microbiome. This is again a feature likely best tailored to the question. However, the choice may be more constrained by practical limitations than for the case of free-living communities. For instance, only very recently did a study carry out sufficient temporal sampling to uncover diurnal oscillations of expression patterns in mice microbiomes (Leone et al. 2015). In humans, daily sampling suggested rapid turnover of the community (Caporaso et al. 2011), yet recently, more reliable sequencing showed that $60 \%$ of species persist on the timescale of years (Faith et al. 2013). This follow-up study has reinvigorated the investigation of stable communities, and serves as a lesson that the reliability of the experimental techniques should not yet be taken for granted. The majority of human studies have acquired samples at only a single, or a few, widely spaced time points. Depending on the microbiome under study and the sampling method, frequent sampling can also significantly disturb a microbiome, adding unwanted variability to the measurements. As the methods are refined to be less invasive, however, these undesirable interference effects can hopefully be made minimal.

Another important feature of the microbiome is spatial structure. It is not clear how spatially structured niches will skew the effective interactions between species, but some microbiome habitats are highly spatially structured and insights from ecology suggests these features can play an important role (Hastings 1978; van Nes and Scheffer 2005). The regions of the body are usually studied separately from one another (see Pedersen and Fenton 2007 for an exception) and spatial features differ a great deal between them. Consequently, comparisons between 
microbiome networks across regions of the body show they can be quite different in their composition and structure (see Figure 1). For instance, vaginal and skin communities tend to have lower alpha-diversity than gastrointestinal-related communities, and vaginal communities in particular are heavily dominated by only a few genera (see Figure 1; Jordán et al. 2015; Zhou et al. 2014). Currently, the application of spatial ecology to studying WH systems and infections is very uncommon, even though it is very clear that some invasions require a spatial ecology approach. There is also growing evidence for the fact that resident taxa move around between local body sites, i.e. translocate, much more than originally thought (Balzan et al. 2007). In addition, spatial structure may also play an important role in structuring the microbial community within a given body-site. For example, patch dynamics are likely to affect the outcomes of human papillomavirus infections (Murall et al. 2014) and possibly most skin or mucosal infections. Also, continuum models seem appropriate for sites containing biofilms, but there are few examples of this approach (though see D'Acunto et al. 2015). Complementary to sequencing approaches for providing both high temporal and spatial resolution, in vivo imaging of the microbiome is coming available (Geva-Zatorsky et al. 2015). Currently limited to animal models, and to a small handful of microbial species, this approach can reveal the effects of an infection in great temporal and spatial detail. Overall then, there is ample room for spatial ecologists to help study how this important phenomenon affects $\mathrm{WH}$ communities and their dynamics.

A

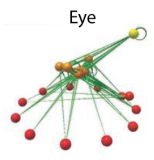

Gasto-intestinal

Gasto-intes
Tract

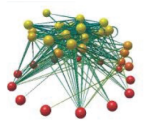

C

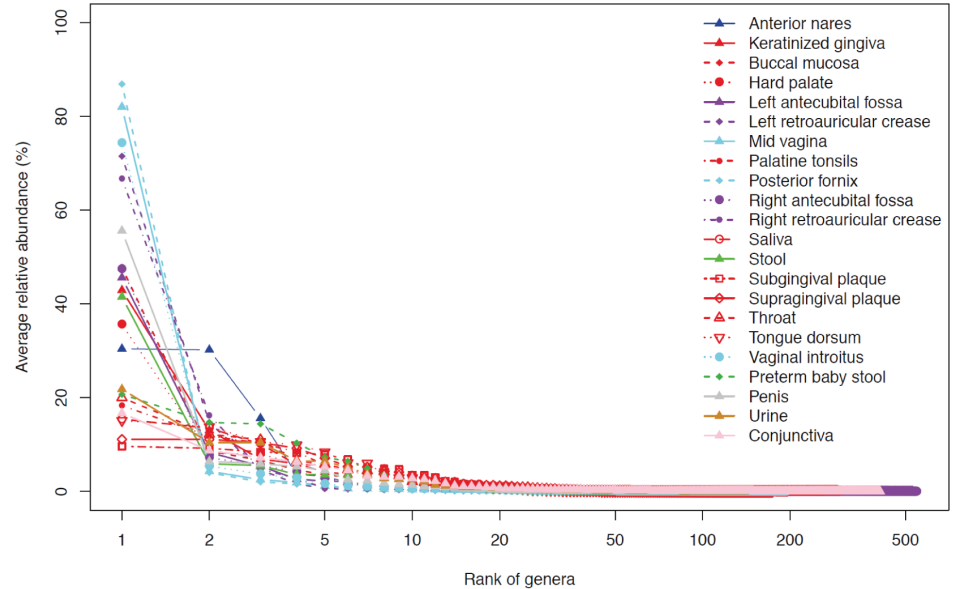

B

\begin{tabular}{|l|c|c|c|c|c|c|}
\hline network & nodes & edges & density & diameter & APL & transitivity \\
\hline anterior nares & 618 & 72136 & 0,3784 & 2 & 1,6216 & 0,4709 \\
\hline buccal mucosa & 1064 & 160541 & 0,2839 & 2 & 1,7161 & 0,3747 \\
\hline hard palate & 1281 & 236289 & 0,2882 & 2 & 1,7118 & 0,3872 \\
\hline palatine tonsils & 1356 & 324713 & 0,3535 & 2 & 1,6465 & 0,47717 \\
\hline saliva & 1560 & 326758 & 0,2687 & 2 & 1,7313 & 0,3826 \\
\hline tongue dorsum & 1438 & 404466 & 0,3915 & 2 & 1,6085 & 0,5194 \\
\hline throat & 1342 & 318287 & 0,3537 & 2 & 1,6463 & 0,4737 \\
\hline stool & 1254 & 293541 & 0,3736 & 2 & 1,6264 & 0,4314 \\
\hline posterior fornix & 462 & 49518 & 0,4650 & 2 & 1,5350 & 0,7040 \\
\hline vaginal introitus & 493 & 59645 & 0,4918 & 3 & 1,5082 & 0,6700 \\
\hline mid vagina & 466 & 62086 & 0,5730 & 3 & 1,4270 & 0,7368 \\
\hline
\end{tabular}

D

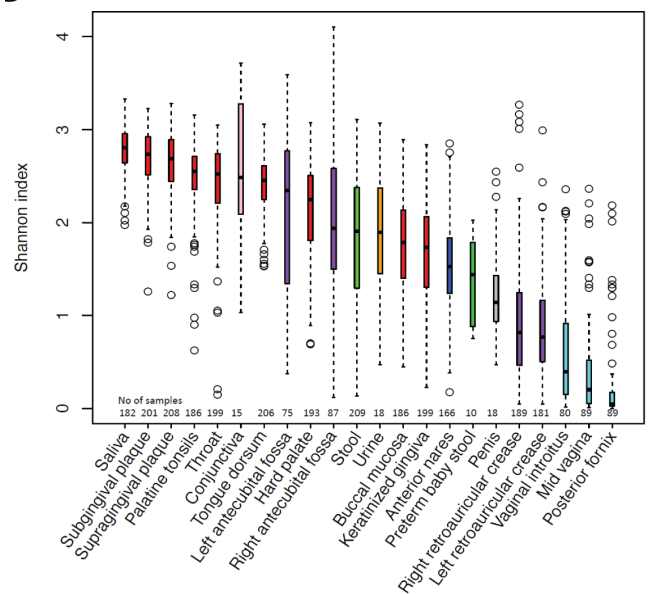

Figure 1. 
In analogy with free-living ecosystems, the notion of a baseline (existing prior to the invasion) and its measurement need to be established in order to quantify the effects of an invasion on the resident network. A multi-stability of the community dynamics (known as alternative stable states in ecology) hypothesizes that the structure of the community dynamics induces multiple basins of attraction in the space of all possible community states. In the absence of strong external perturbations, communities found in a given basin tend in time towards the corresponding stable state as the local attractor of the dynamics. In support of the stable community hypothesis, studies have shown that the communities in healthy individuals indeed cluster into a handful of types (e.g., vaginal microbiome states, Gajer et al. 2012, and enterotypes of the gut, Arumugam et al. 2011, that persist over time, Faith et al. 2013). Nevertheless, there are reports (Gajer et al. 2012) of community dynamics with fast-fluctuating species abundances such that the identities of the prominent species undergo quick turnover. This kind of short correlation time dynamics, reminiscent of chaos but not necessarily chaotic, was nevertheless found only in healthy individuals. This is evidence against the hypothesis that such dynamics is pathological for the host and suggests that long-term maintenance of a given set of species may not be necessary for normal, healthy functioning. Regardless of the relevant timescale of microbiome dynamics, sampling and sequencing the microbiome at multiple time points provides the means to establish a baseline from which the effects of an invasion can hopefully be observed. We note that other kinds of measurements, for example of environmental properties such as $\mathrm{pH}$, can also be informative across an invasion event (e.g., Gajer et al. 2012).

\subsection{Measuring the microbiome}

The maturing revolution in sequencing techniques has focussed attention on the characteristics of the microbiome accessible by methods from the three -omics: genomics, transcriptomics, and proteomics, providing information on DNA, RNA, and proteins, respectively. Respecting the terminology, the prefix 'meta-' should be prepended to these terms since, in microbiome research, they are applied to samples containing multiple species. DNA sequencing is often used to identify the species present. The number of reads of a given sequence can also be used in principle to infer cell counts. Primers can amplify different genes at different rates, and initial primer concentration should be carefully controlled so as not to introduce additional bias (Brooks et al. 2015; Kennedy et al. 2014; Tremblay et al. 2015). Which proteins are being expressed can in principle be obtained from RNA sequencing. It has become apparent over the last decade, however, that expression level does not reliably report the actual amount of protein present (Rogers et al. 2008). For this, protein purification using antibody assays are often used. Moreover, since an altered state of a protein often signals the activation of its function, current proteomic research is focussing on methods for identifying alternate, e.g. phosphorylated states of the same protein complex (Aebersold and Mann 2016). More advanced sequencing methods seek to account (via, for example, molecular barcoding) for artefacts due to the amplification, such as copy error and primer bias (Best et al. 2015). Reliable or at least soon-to-be reliable estimates of abundances can be obtained with these methods. The abundances are nevertheless often taken relative to the sample size so that data from multiple samples can be 
compiled. This operation can lead to spurious correlations between species abundances that can, and should, be corrected (Berry and Widder 2014).

\subsection{Inferring the interaction network}

The inference of human microbiome interaction networks, in particular from metagenomic sequencing data, has become a preoccupation of many in the field. Doing so accurately will likely be required before understanding the effects of invasions on the microbiome community. These methods employ the abundance data from samples taken either from the same community at multiple time points, or from a single sample from the same body-site across various hosts, or in the best case both.

In a first approach, researchers analyse pairwise correlation matrices of taxa abundance, drawing a link between pairs of taxa whose pairwise correlation surpassed some threshold value. For instance, co-occurrence networks (also called association or correlation or coexisting networks; Vacher et al. 2016) are usually built on statistically significant co-presence or co-absence of pairs of taxa. Taking such networks as indicative of ecological interactions is problematic, however, as co-existence patterns, such as presence and absence, are not a direct result of pairwise ecological interactions, such as mutualism and competition, respectively. They are shaped in non-trivial ways by the type of underlying interactions, habitat use and abiotic factors. Analysis of the approach has nevertheless framed the technical difficulties of the problem (see Faust and Raes 2012 for a review and Marino et al. 2014 for a recent example). Recent studies show that these correlation-based networks perform poorly at recovering the actual interaction network from synthetically produced data (Berry and Widder 2014; Fisher and Mehta 2014; Kurtz et al. 2015).

In performing analysis on sequence data, the often widely varying sample sizes and primer bias require first transforming the data. The most common transformation is simply to take relative abundance, but this introduces a sum-constraint making inadmissible many statistical inference approaches. The appropriate statistical theory for such compositional data was communicated to the field in Kurtz et al. (2015), in which the data is transformed to centered log-ratios. Kurtz et al. (2015) go on to infer a network of associations by fitting a probabilistic graphical model to this transformed data. Such models are based on conditional dependencies and so can in principle distinguish co-existence patterns due to direct ecological interaction and those due to spurious correlation. An added regularization term to select for sparse solutions allows for the problem to be solved efficiently, and they test the method on data generated in silico. The method's performance depends on some network properties, performing less well for larger maximum indegree (the number of links going into a given node; see next section for a description of other network properties), and relies on the number of connections not scaling faster than the number of taxa (i.e. it may fail for densely linked microbiomes). 
Another more ambitious class of approaches to inferring interaction matrices seeks to leverage the temporal correlations in the data. Such methods must handle both the deterministic and stochastic contributions to the observed time series. One subset of methods in this class use causality measures computed on time series to determine the presence and direction of a causal interaction between two species. Sugihara et al. (2012) is an example combining the stochastic prediction of Granger causality with the deterministic embedding of attractor reconstruction. The high-dimensionality of sequence data can apparently help overcome the finite sample limitations of the latter (Ye and Sugihara 2016). A more standard approach is to use a noise model for the observed abundances at each time point to extract a mean abundance, whose time series is then fit by deterministic models of the underlying community dynamics. The hope is that the temporal correlations in the dynamics provides additional information about interaction strengths allowing some of the noise issues in the measurements to be overcome and give more reliable results. Moreover, if the chosen model of the dynamics is sufficiently faithful to the real system, then the values of the inferred parameters (e.g. growth or attack, rates) actually reflect the ecology of the system. Generalized Lotka-Volterra (gLV) models are often used with an additional species-specific, time-dependent term representing disturbances. This type of approach has been pursued in a number of recent publications and there are a growing number of software packages available online, most notably MDSINE (Bucci et al. 2016; also see Eq.1 below and Figure 2). A detailed comparison of these packages (by performance, accessibility, versatility, etc.) goes beyond the scope of this review, but would be a welcome contribution to this growing body of work. Here, we briefly discuss some of the major issues and proposed solutions. 
A

$\alpha_{i}$

Growth

0.60 .91 .21 .5
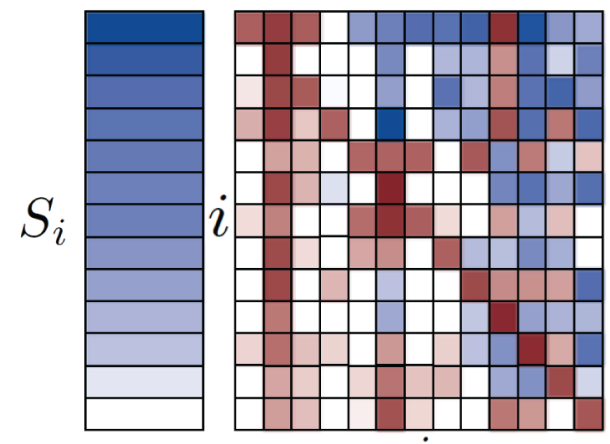

$j$

C

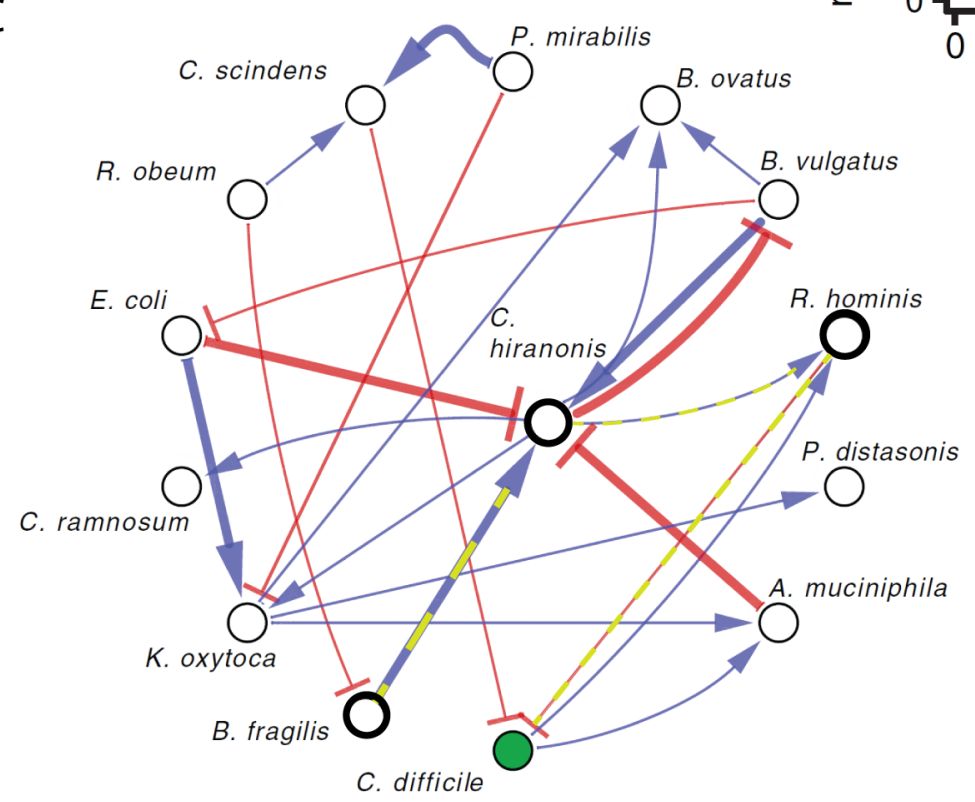

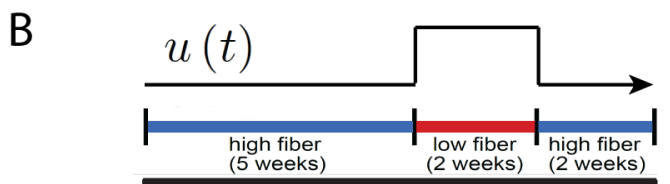

$-4-20 \begin{array}{lll}2 & 0 & 4\end{array}$
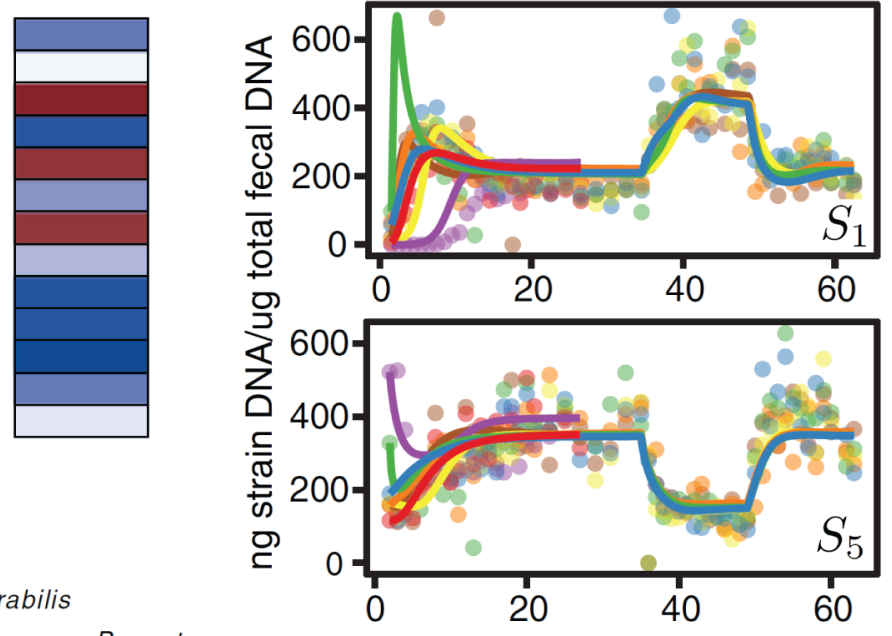

Days

Figure 2.

The MDSINE package is a suite of methods. At each time point, they infer the mean of a probabilistic model of read counts to the abundance data, connecting means across time points using splined functions of time. The unbound growth, interaction, and disturbance parameters of a gLV model are inferred on this splined representation of the data using gradient matching and also with a regularization to overcome the undersampling. Relevant to invasion analysis, the quality of the inference is increased when applied to data sets obtained over periods of stepwise disturbances. Such cases allow the dynamics to exhibit its timescales making the data more 
informative to parameter estimation, and ultimately network reconstruction. The authors state that there was no clear relationship between the reconstruction performance and the in-degree. This benefit over the inference methods of Kurtz et al. (2015) and the literature cited therein could reflect the utility of dynamical information in this inference problem. More work is needed to settle the origin of this discrepancy.

Many studies test these methods on synthetic data. For example, the inferred matrices of Fisher and Mehta (2014) have correlations of 0.8 with the original interaction matrix used to simulate the data. How this number depends on the form of the community dynamics was not investigated however, and different functional responses (i.e., types I,II and III; Holling 1959) in the community dynamics equations may play a role in the strength of the correlation between the ground-truth and the inferred network. Unlike in nervous systems where the ground-truth synaptic connectivity between neurons can at least in principle be obtained empirically, there is currently no obvious definition or way of obtaining ground-truth connectivity in a real microbiome. Nevertheless, similar methods to infer parameters of the underlying network using dynamical models are being applied to other microbial networks, such as the bacteriophagebacteria networks (Jover et al. 2013). With inference algorithms and machine learning developing at a fast pace, more work is needed to find more reliable and general methods for inferring microbiome interactions

Once links are reliably inferred, interaction networks can be analysed with graph theoretic techniques. Simple measures can help distinguish nodes with atypical properties. Highly connected nodes, such as Bacteriodes stercoris and B. fragilis (Fisher and Mehta 2014), may indicate the species on which the community's stability depends, in analogy with the keystone species concept from ecology (discussed more explicitly below in Section 5).

Beyond single species, but before considering the community as a whole, systems of a handful of species nodes can be studied. When connected in particular ways and with classic interacting population dynamics. These are called modules in ecology (e.g., the diamond module; Holt et al. 1994), and each provides characteristic dynamical behaviours (reviewed in McCann and Gellner 2012). Ecological modules have been identified and studied as a first step to understanding larger community networks that contain them as sub-networks. Ecological modules have been found to also appear in WH systems, particularly in pathogen-host interactions (see Figure 3). Similarly, in the context of the microbiome, Stein et al. (2013) performed inference with a dynamical model and found particular circuits of species that are involved in successfully repelling an infection by $C$. difficile.

For large, or many replicates of, interaction matrices, one can employ some of the many wellestablished graph statistics (Newman 2003). These include how dense the network is (connectance); how many links 'wide' the network is (graph diameter); the degree of modularity (not to be confused with ecological modules), i.e. link prevalence in given clusters compared to link prevalence between clusters; the degree of clusteredness, i.e. how often neighboring species with one species are also neighbors with each other, etc. (see Table 1 and David (2017) in this issue for a review of network properties and invasions). One can also calculate 
motif statistics, i.e. the over- or under-representation relative to chance of small groups of nodes connected in particular ways (but agnostic to their dynamics). On the other hand, one can incorporate information extrinsic to the graph, and for instance intrinsic to the nodes. For example, OTU assortativity is the propensity of connections between nodes with similar 16S rRNA profiles. The inferred gut networks in Kurtz et al. (2015) displayed a high degree of OTU assortativity. However, overall, very few studies have directly investigated motifs in hostassociated microbiomes and most of what has been done is in metabolic network studies (Table 1).

Assessing the over- or under-representation of motifs relative to the prevalence expected by chance can be used to analyse microbiome abundance time series. With datasets sampled at a high enough temporal resolution, the possibility to classify their trajectories arises. Once sufficiently long time-series can be measured, comparisons of cross correlations of the abundances from microbiome communities across body sites could provide signatures of leaders and laggards in the whole body microbiome dynamics. Finally, we mention that network reconstruction competitions based on benchmark datasets can be a fruitful way to attract highperforming algorithms (e.g., Orlandi et al. 2014).

\subsection{Theoretical microbiome invasion dynamics}

There is a wealth of theoretical ecology literature using dynamical systems theory to study population and community dynamics. The vast majority of existing models and theories that have taken this approach to $\mathrm{WH}$ invasions are found in the pathogen literature, more specifically the literature related to multiple infections (Read and Taylor 2001). These works normally do not include commensal phyla (for an exception see Lysenko et al. 2010). Generally, in this WH disease modelling framework, the host is abstracted as populations of resources (usually cells) and immunity cells. Resident species in this case are acute or chronic infections already established in the system. Invasion analysis is done to see if a second invader with new traits, or another species altogether, can take hold. These models have been very successful in helping study viral-immunity interactions (Perelson 2002), studying viral or bacterial WH evolution (Luciani and Alizon 2009) and aiding in antiviral and antibiotic treatment studies (Koizumi and Iwami 2014). Attempts to capture microbiota interactions mathematically have not focussed on feeding relationships, as is the case for pathogen-immunity models and free-living ecological systems. Microbiome network models, in contrast, define links and their weights by effective cooperative and competitive interactions between species (see Eq. 1 below).

An important ecological insight that has been found using the dynamical systems approach is that many ecological modules found in free-living systems are also found inside the body (Fenton and Perkins 2010; Holt and Dobson 2006; Murall et al. 2012; see also Figure 3). With the inference of networks from sequencing data, we are only now beginning to see attempts at finding what modules are important to microbiome communities (Table 1), and particularly how they can help explain community stability in face of invasions (see analogous work for free-living systems and biological control; Holt and Hochberg 2001). 


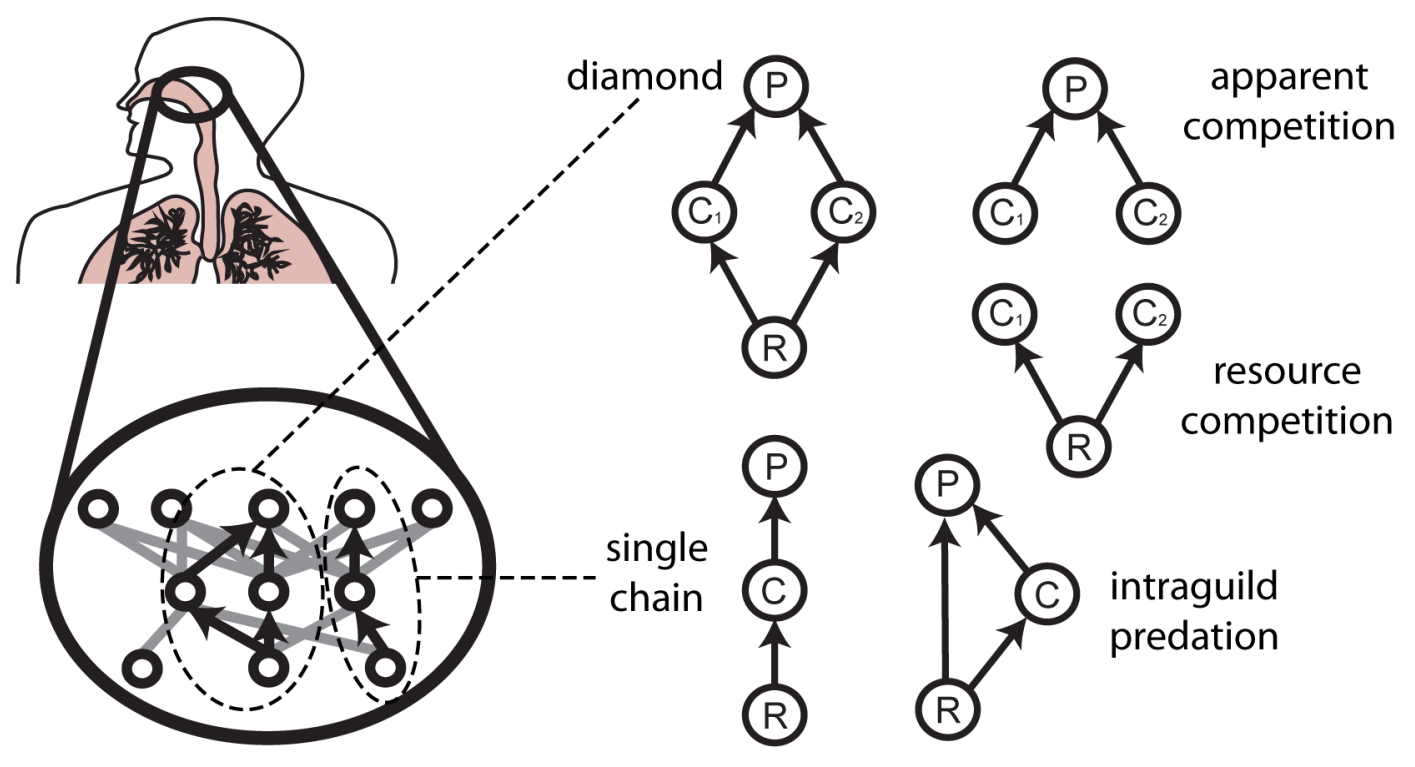

Figure 3.

Given that the field has been rightly preoccupied with inferring networks and interactions, it is not surprising that despite a considerable amount of discussion about microbiome community stability (e.g., Bäckhed et al. 2012; Hunt et al. 2011; Lozupone et al. 2012; Relman 2012; Romero et al. 2014), the majority of human microbiome models have not yet focused on stability and resilience to invasions. A recent exception for stability analysis was presented by Coyte et al. (2015) who used a classic theoretical community ecology approach to study microbial community stability. This approach focusses on the stability properties of interaction matrices arising from community dynamics models such as a system of Lotka-Volterra-like population dynamics, a phenomenological population network model that can incorporate multiple types of interactions. Such models can incorporate invasions to the system by the addition of the appropriate equation for the invader's population dynamics. The interaction strengths with the rest of the community can be turned on at the desired time of invasion. Another kind of community perturbation is a disturbance incorporated as a time-dependent change in the affected parameter(s). The dynamics of a community of $N$ species, $S_{i}, i=1, \ldots, N$, interacting via a type I functional response, and which, for the purposes of example, experience a timedependent disturbance in their growth rates can be written as:

$$
\frac{\mathrm{d} S_{i}(t)}{\mathrm{d} t}=\left(\alpha_{i}+\gamma_{i} u(t)\right) S_{i}(t)+\sum_{j=1}^{N} J_{i j} S_{i}(t) S_{j}(t)
$$

Here, $J_{i j}$ is a matrix, the elements of which are real numbers corresponding to the interaction strength with which species $j$ affects species $i ; \alpha_{i}$ is the basic growth rate of species $i$; and, in a simple example of a disturbance, $\gamma_{i}$ is the constant amount of change in the growth rate induced 
by the binary-valued function, $u(t)$, denoting whether the disturbance is present or absent at time $t$. Such a system has been effectively used to infer interactions (Figure 2; Bucci et al. 2016). Mathematical systems of this form allow for pair-wise interactions, such as competition ($/-)$, mutualism $(+/+)$, predation, herbivory or parasitism $(-/+)$, amensalism $(0 /-)$, commensalism $(0 /+)$, and no interaction $(0 / 0)$. These equations in principle even allow for trophic levels, such as predation (e.g. protists or the immune system) or parasitism (bacteriophages), through particular block structures in the matrix, $J_{i j}$ (see Johnson et al. 2014 for such an example using the matrix approach outlined next).

The large number of interactions in a diverse ecosystem makes the determination of elements of $J_{i j}$ impractical. An alternative approach, the so-called matrix approach pioneered by Sir Robert May, specifies the interaction matrix only through the statistics of its elements. Results from random matrix theory can then be utilized to determine the average stability properties of the stationary states of the corresponding dynamics as a function of the remaining parameters in the model (May 1972). Applying this approach to microbiome systems, Coyte et al. (2015) found results consistent with previous ecology research, namely that synergistic interactions are destabilising and weak interactions are stabilising. Gibson et al. (2015) employ a similar model to study the effects of one-time perturbations that knock the community between some small number of states.

Reconstructing interaction matrices before and after an invasion may not be necessary if only the resilience of the network is of interest. For example, a recent work shows that a particular reduction of high dimensional dynamics onto a single effective dimension highlights a resilience function (how abundance decays as a function of some kind of loss) depending only on the form of the equations for the dynamics and not on the values of the system parameters or perturbations (Gao et al. 2016). In a bipartite network of plants and pollinators, they show that the form of the decay of the average population sizes as a function of the number of nodes or links lost, or the reduction in interaction weight, all collapse onto the same bifurcation diagram. This diagram shows how changing an effective parameter beyond a critical value leads to a change in the steady state from one in which species have relatively high abundance to one where they have low abundance.

Despite the seeming ease with which ecological theory can be transferred over to the microbiome, there are caveats. Creating a consumer-resource modeling framework for the microbiome is less straightforward than in free-living systems, since microbes interact in unique ways such as cross-feeding and have complex nutrient use, and thus energy flow (Foster et al. 2008). We know from free-living systems that the destabilising effect of positive feedback in a community's dynamics can be controlled by the stabilising effects of predation, whose exploitative interactions then maintain the community's stability. The presence of microbial eukaryotes (i.e., protists; Parfrey et al. 2011) or predatory bacteria (even if they appear in low abundances) in host-associated environments are then likely important, but for now are understudied. Multi-trophic interactions, for example, are much more investigated in other, e.g. terrestrial, microbial systems (see Saleem 2015 book for review). 
There are few other studies on perturbations in microbial systems, so we briefly review some of them here. Allison and Martiny (2008) discuss microbial community stability to disturbances; D'Acunto et al. (2015) model multispecies biofilms and their invisibility; and in a recent study, Peura et al. (2015) numerically test the response of microbial networks to the removal of taxa. Indeed, some important properties of ecological networks, such as the pervasiveness of weak interactions instead of strong interactions (McCann 2000) remain unstudied in human microbiome communities. Yet, the increasingly network-oriented approach to studying the microbiome lends itself to investigating these sorts of properties and how they mediate stability and resistance to invasion (Foster et al. 2008). In Table 1, we highlight some key findings of microbiome network properties and how they may help understand community stability.

Finally, we mention there are even fewer examples of attempts to create full networks of interacting immune cells, pathogens and resources. Griffiths et al. (2014) assemble a network of such interactions, drawing links between pairs of species on which some association has been published. Analysing the resulting three-trophic layer network, they argue that WH pathogen interactions tend to be predominantly indirect; that the network has several identifiable and repeated motifs; that most nodes have less than 10 links, i.e. degrees (nodes are connected to few other nodes); and that there is bottom-up control (the community dynamics is driven mostly by nutrient availability Griffiths et al. 2014). Unfortunately, this analysis did not include resident taxa (e.g., microbiome) which may change these results; nor did they study how the model responds to perturbations.

In conclusion, there is a large body of work in theoretical ecology that can likely be leveraged in investigations of the microbiome. Nevertheless, there are important distinctions and at this early stage, it would be wise to keep model development close to data. Fruitful future research will likely come as WH disease modelling begins to include more of the resident community (or at least important properties of these communities) and as microbiome modelling begins to apply invasion analysis. An exciting step possible when these other directions have matured will be to include the host resource and immune cells to see what level of control they can enact. With all this accumulated knowledge, we expect that models of the stability of WH systems will eventually inform the design of perturbative experiments of the microbiome, not only in microbiome science but also for human health.

\section{Unique features of within-host communities}


While there are many similarities between WH and free-living ecosystems, there are several key features unique to $\mathrm{WH}$ biological invasions that affect their interaction with the host microbiome. One of the most important distinctive features, with regards to invasions, is the immune system, which is itself an interacting network of cell populations thought to be primarily specialized for defending the host (and by proxy its contained microbiome) against pathogens. Not surprisingly then, it also plays a role in managing the $\mathrm{WH}$ community composition and dynamics. We also discuss two additional unique features of $\mathrm{WH}$ communities in this section, namely invaders that are fatally virulent to the host, and interactions among resident species and with their host that can prevent invasions.

\subsection{The immune system}

Research establishing the degree of trophic separation in $\mathrm{WH}$ microbial systems has identified the immune system as an important trophic level of top predators. In fact, a recent hypothesis on the origins of the immune system points out the similarities between immunity (e.g. phagocytosis and enzymes) and digestive properties and functions (Broderick 2015). While the population dynamics of ciliates and predatory bacteria are likely to have many similarities to free-living predators (e.g., similar feeding functional forms), the immune system has several features that depart from this analogy. For instance, the set of components of the immune system active in the response to a pathogen invasion is itself a network made up of populations of cells that communicate with each other, via populations of helper cells and signalling compounds (e.g., cytokines). This degree of cooperation between predators, a consequence of a shared genetic trajectory, is not something that is observed in free-living systems. While there are parallels of generalists and specialists to innate effectors and adaptive effector cells, respectively, these effectors engage in collective behaviours that go beyond free-living systems, which we discuss in the following two paragraphs. Studying the population dynamics and evolution of this system is itself a growing field to which ecology and evolutionary biology have been applied (Alizon and van Baalen 2008; Mayer et al. 2016; Murall et al. 2012; Pilyugin and Antia 2000; Sadd and Schmid-Hempel 2009; Wodarz 2006). To close the section, we discuss the ways in which the $\mathrm{WH}$ community coordinates with the immune system in countering a pathogen invasion.

\subsubsection{Host barriers and the innate immune response}

The protection provided by physical barriers in the body is one of the fundamental forces shaping microbial communities, as they dictate which microbes interact. Hosts have constitutive defense systems, such as the skin barrier (e.g., cornified cells, surface fluids, etc.; Sanford and Gallo 2013) that are fundamental in keeping unwanted invaders out or trying to prevent movement around the body. Bacteria that have the ability to translocate out of their primary niche to invade new body sites often produce pathogenesis (Ribet and Cossart 2015). Similarly, pathogenic invaders regularly have traits that help them navigate through these barriers in order 
to spread to various target niches in the body. Overall, then, host barriers are not enough to keep out invasions.

The vertebrate immune system is characterized by two distinct but interacting suites of mechanisms: innate and adaptive responses. Innate responses use general mechanisms that do not target specific pathogens (i.e., the generalist predators), whereas the adaptive responses are triggered by identification of specific antigens (i.e., the specialist predators). The innate response is primarily a first responder system that recognises non-self and aims to keep down early growth of an unwanted invader. The adaptive response is typically most effective at clearing an infection, and importantly, responsible for rapid response to an attempt to re-infect. The innate response actively interacts with the resident microbial community (more below) and important microbicides are secreted throughout the body in order to help prevent infections (e.g., angiogenins in the gut; Hooper et al. 2003). This level of community-wide "border control" is truly a $\mathrm{WH}$ phenomenon.

\subsubsection{Rapidly evolving predation}

Investigations into infectious disease and immunity interactions have found that the immune response can cause dynamics similar to consumer-resource interactions. For instance, coinfecting pathogens that are antigenically similar (e.g., different strains of the same species) experience cross-reactivity, or in ecological terms, apparent competition (Brown and Grenfell 2001; Holt and Barfield 2006; Smith and Holt 1996). Similarly, the stimulation of responses by one pathogen can have a range of top-down impacts on other microbiota in the body (McSorley and Maizels 2012). For instance, helminth infection can down-regulate responses to intracellular pathogens, such as Plasmodium spp. (Graham 2008), and as found in another study, helminth infection can promote a type 2 immunity response that helps prevent Bacteroides colonisation (Ramanan et al. 2016).

Nevertheless, unlike free-living consumer-resource interactions, the predator is evolving rapidly during the course of the invasion, and thus the evolutionary and ecological time scales are simultaneous. Historically, free-living predators where considered to be 'prudent' because they did not evolve to be so highly efficient as to drive their prey extinct (Abrams 2000). Clearly, however, the immune system is able to evolve fast enough to drive invaders to extinction (i.e. clearance). Furthermore, if the invader is also able to rapidly evolve during the course of the invasion, then a co-evolutionary arms race can take place.

The adaptive immune response consists of two main effector populations involved in clearing infections (i.e., ousting an invader): $\mathrm{B}$ and $\mathrm{T}$ cells that are generated with antigen receptors that are highly diverse. Once triggered by specific antigen, they are made effective through an amplification process by which the population of those cells with receptors that bind strongly to circulating antigen undergo accelerated division. Once amplified, they can rapidly deplete a pathogen invasion population. Even more striking is that B cells undergo evolution-like rounds of mutation and positive selection which results in hyper-specialist predation with orders of 
magnitude higher attack rates. Another unique feature is that once an adaptive immune response has mounted and has infiltrated the infection site, the response will often decouple its growth from the presence and density of the pathogen (antigen-independent proliferation program; briefly reviewed in Bevan and Fink 2001). This independent growth allows these potent immune effectors to swamp the area and completely clear the pathogen. Finally, the immune system, builds a library of memory cells in order to aid its recognition of, and speed up its response to, a similar invasion in the future. Overall, WH communities have in the immune system a highly specialized and effective defence against invasions, the main purpose of which is to prevent invaders from colonising, establishing and spreading within the body.

\subsubsection{Immunity controls community composition and dynamics}

An important part of the host immune system's ability to recognise self versus non-self is due to the family of proteins called the major histocompatibility complex (MHC) and the immunity cells that use them to create a response (e.g., T-helper cells). As the most diverse region of the entire genome, the multiple genes that code for these proteins dictate the immunological repertoire of what the host can recognise. The diversity of this complex plays a central role in the immune system of vertebrates, whose slow life histories might otherwise leave them at a severe disadvantage in the co-evolutionary arms race between microbial invaders with short generation times and large population sizes (Hamilton 1980; Jaenlke 1978; Lively 2010; Sommer 2005). Interestingly, animals, including humans and non-human primates, have been shown to choose mates based on cues that signal high MHC diversity; however, they do not always choose mates with dissimilar repertoires (which would, in theory, provide the benefit of increased immunological repertoire for their offspring; reviewed in Winternitz and Abbate 2015), thus suggesting that optimal MHC diversity is not always maximal MHC diversity (Milinski 2006; Wegner et al. 2003). One speculation, as to why this may be, is that too much MHC diversity may disrupt positive associations between the host and its microbiota. An experiment in threespined sticklebacks has demonstrated that fish with higher MHC diversity have lower diversity in their gut flora, and that different $\mathrm{MHC}$ allele repertoires are associated with shifts in the relative abundance of some bacterial functional groups (Bolnick et al. 2014). Recently, Kubinak et al. (2015) found that the role of $\mathrm{MHC}$ in regulating gut microbial community composition is also applicable to mice, in which specific MHC genotypes reduce the fitness of resident microbiota, favouring systemic infection.

Evidence that the immune response affects resident microbiota composition also comes from the examination of cases where immune function is compromised. From studies of infectious diseases where immune-deficient patients are often unable to limit the number of infections, one could think that a less effective immune response would allow a resident microbial community to be more diverse. However, studies like Oh et al. (2013) show otherwise. Patients with various immunodeficiencies were found to have decreased skin microbial diversity as well as different relative abundances of taxa in comparison to their healthy counterparts. Interestingly, these altered microbiota compositions were also associated with increased susceptibility to infectious diseases or opportunistic pathogenic fungi. This correlation is also likely to be causal, as Taur et 
al. (2012) showed that temporary pre-transplant removal of circulating granulocytes and monocytes in allogeneic hematopoietic stem cell transplantation patients leads to reduced gut microbiota diversity, followed by invasion and establishment of pathogenic species that compromise the patients' clinical outcomes. Of course the elevated incidence of pathogenic bacteria or fungi in these communities would need to be teased apart from the impact of the immunodeficiency itself; however, the diminished resident bacterial diversity is likely to also play a role. In a telling example, non-functional immune response to the flesh-eating protozoan parasite Leishmania major in germ-free mice is rescued by the addition of the skin commensal Staphylococcus epidermidis, suggesting that this resident bacteria aids local inflammation and thus boosts T lymphocyte function (Naik et al. 2012). These studies suggest that the immune system plays a complex role in engineering the $\mathrm{WH}$ community allowing or actively promoting a more diverse community composition (which is potentially more stable and less invasible).

\subsection{Resident species actively prevent invasions}

The direct thwarting of a pathogen invasion by members of the resident microbiota, termed colonisation resistance, is a phenomenon seen in various regions of the hosts' body. This relationship is similar to symbiotic relationships seen in plants, e.g. ants on acacia plants, and is thus a common host defense strategy. However, non-microbial free-living ecological networks typically do not have these kinds of invasion prevention mechanisms that are community-wide.

Resident bacteria can interact directly with host cells to help prevent or clear an infection. For instance, the skin resident species $S$. epidermidis interacts directly with host keratinocytes to inhibit infection of pathogenic $S$. aureus and group A Streptococcus species by triggering keratinocyte expression of antimicrobial peptides (AMPs). This resident species also produces phenol-soluble modulins that work with the AMPs to improve killing efficacy, and in addition they engage in signalling that helps keratinocytes cells repair and survive during infections (Grice and Segre 2011). In fact, AMPs are produced throughout the body and so these host-resident interactions are probably more common than may be currently appreciated. The gut microbiota is also known to engage in similar relationships with host endothelium to help maintain homeostasis necessary for organ functioning. Notably, gut disease states such as inflammatory bowel diseases (e.g., Crohn's disease) are linked to reduced production of antimicrobial defenses (reviewed in Ostaff et al. 2013; Buffie and Pamer 2013).

In addition, resident bacteria directly interact with one another to prevent invasions. For instance, healthy female genital tracts are colonised by lactic-acid bacteria, mostly Lactobacillus spp. These bacterial species are ubiquitous and found in various hosts (as diverse as mammals and birds) and in various organs (gut, mouth, female genital tract, breast milk). Numerous Lactobacillus spp. have been described in healthy human genital tracts and the most frequent and abundant species are L. crispatus, L. gasseri, L. jensenii and L. iners (Ravel et al. 2011; Gajer et al. 2012). The key protective roles of Lactobacillus spp. are usually attributed to (i) their lowering of environmental $\mathrm{pH}$ through lactic acid production, (ii) their production of bacteriocins and other bacteriocidal compounds such as biosurfactant or hydrogen peroxide $\left(\mathrm{H}_{2} \mathrm{O}_{2}\right)$, and (iii) their competitive exclusion of other bacterial species (Boskey et al. 2001; Fayol-Messaoudi et 
al. 2005; Kaewsrichan et al. 2006; Ling et al. 2013; Ravel et al. 2011). Similarly, a study on the persistence of human-associated Bacteroidetes in the gut (discussed earlier in Section 2.3) showed that some bacterial species can exclude an invader by producing bactericidal effectors triggered by direct physical contact, and that this activity is mediated by resistance evolution and relative population densities between species (Wexler et al. 2016). Finally, how our commensal or mutualist viruses interact with each other or the microbiome in order to prevent invasions is significantly less understood. An example reviewed in Clemente et al. (2012) showed that infecting mice with a latent gammaherpesvirus (a virus highly related to common childhood infections in humans, such as Epstein-Barr virus and Human cytomegalovirus) seems to increase resistance to bacterial infections, at least for a period of time (Yager et al. 2009). More research into the human virome and its effects on invasions is greatly needed.

To conclude, it is tempting to anthropomorphise the mutualistic interaction between humans and their microbiota with the simplification that we provide food and shelter while microbiota prevents invasions. We caution that, in fact, invasion prevention mechanisms are often not for our direct benefit, since some may be secondary or indirect effects. Indeed, many protective effects are actually indirect effects via the immune response, and, in addition, the mutualistic effect of a resident can come with costs. For instance, chronic infections by Helicobacter pylori seems to confer some protection against tuberculosis (Perry et al. 2010), but chronic infection of $H$. pylori is also associated to other pathogenic effects (e.g., increased incidence of gastric cancer). Likewise, several viruses and bacteria are mainly commensal, but persistent carriage of them can lead to pathogenic effects (e.g., the oncoviruses such as Merkel cell polyomavirus, Epstein-Barr virus and high-risk Human papillomaviruses). Some recent, interesting microbiome results are similar to earlier results on multiple infections showing that pathogens can have pleiotropic effects by protecting their hosts against other (more virulent) pathogens (Michalakis et al. 1992). As a counterexample, a study by Stecher et al. (2010) found that the presence of related bacteria in the resident microbiota can facilitate bacterial invasion. Overall, then, we do not have the full picture of this commensal-pathogenic continuum. As such, we echo the suggestion by Mushegian and Ebert (2016) that the term "mutualism" be used more sparingly, i.e. only when there is a clear pairwise interaction or when mutual reciprocity has been directly investigated and demonstrated.

\section{Box. 1 - Does relatedness help or hurt a species' ability to invade?}

[[ Box 1 here ]] 


\subsection{Fatally virulent invaders}

Virulent invaders have negative health consequences on their hosts (e.g., morbidity or mortality), and some WH invaders can be so pathogenic that they kill their host. This would be equivalent to a free-living invader completely wiping out all biological life in the area it invades. In free-living systems, biological invasions can have very strong destabilising effects but rarely do they directly eliminate the entire community. There is clearly a cost to the invader to completely kill the ecosystem it grows and lives in, and thus there is a great deal of literature dedicated to understanding the evolutionary forces that maintain this costly trait.

In general, pathogen virulence is expected to be counter-selected because killing the host decreases the duration of the infection and, hence, the epidemiological fitness of the pathogen. This led to the avirulence hypothesis, which postulates that given enough time, host-pathogen interactions should evolve to be benign (Méthot 2012). The same intuition applies to the human microbiota and its invaders: virulence should be counter-selected. Over the years, it has been realised that the avirulence hypothesis was at odds with the data (see for example Ball 1943). There are several explanations as to why virulent strains can persist and they broadly divide into non-adaptive reasons and adaptive reasons (Alizon and Michalakis 2015). The former reasons imply that the host is not involved in the transmission route of the pathogen or, more generally, that virulence does not affect pathogen fitness so there is no selection against it. Virulence can persist if it confers an advantage in the between-host life-cycle of the pathogen. The classic perspective is that more virulent strains have a higher transmission rate. A more appropriate approach consists in measuring the pathogen between-host fitness as a function of its virulence (Alizon and Michalakis 2015).

Another explanation for virulence, that is arguably more pertinent in a microbiota context, stems from within-host interactions. Microbes compete for resources inside their host (Mideo 2009; Smith and Holt 1996), either via direct competition for nutrients (such as iron) or indirect competition (such as the apparent competition via the immune response). It is usually expected that the most virulent strains are the most competitive, as proven experimentally in the case of Plasmodium chabaudi (de Roode et al. 2005). However, this result strongly depends on the type of within-host interactions (Alizon et al. 2013). For instance, if pathogens interact by competing for public goods they produce, then the less virulent strain wins the competition, as demonstrated by Pollitt et al. (2014) using quorum-sensing in bacteria. Overall, this means that a more or a less virulent invading strain can be favoured depending on the nature of the interactions in the microbiota (Lysenko et al. 2010). Furthermore, predicting the direction of evolution in multiple infections caused by different species is even more difficult given that it requires coevolution models. In addition, models of zoonotic or opportunistic infections - which is often the case for pathogens invading the microbiome - must consider the possibility that the host is not part of the parasite's life cycle, which means that there will be no direct selective pressure on the traits expressed in this host type as long as it remains an evolutionary deadend (Brown et al. 2012). 
A final adaptive explanation for the persistence of virulent strain is within-host evolution. In other words, these strains can be completely maladapted to the between-host level (to the point that they are rarely transmitted), but they keep evolving de novo over the course of an infection because they are well adapted to the within-host level and evolution is short-sighted (Levin and Bull 1994). One of the best illustrations of this is provided by experiments using Haemophilus influenzae in mice (Margolis and Levin 2007). These bacteria are usually commensals harboured in the nasopharynx but in some cases they can cause an invasive disease by colonising the bloodstream. By using tagged bacteria isolated from the blood and the nasopharynx of infected rats, the authors showed that the bacteria's diversity is lower in the blood than in the nasopharynx, suggesting a bottleneck occurred during the colonisation of the blood. Using these remaining bacteria to re-infect new mice, they found they could induce the disease. This example of $\mathrm{WH}$ evolution suggests a context-dependence of a bacteria's virulence, in which context may well include other bacteria. The within-host evolution literature, which dates back at least to the 1970s with articles on cancers as evolutionary processes (Nowell 1976), is interesting as a starting point to import evolution ideas into the microbiota context because it may offer an adaptive explanation to virulence without involving fitness at the between-host level, which is complicated to measure due to the difficulty in determining the life cycle of the microbe and due to epidemiological feedbacks (i.e. the fact that the outcome of an infection may be affected by processes occurring at the epidemiological level, such as a new parasite invading the host). Of course, there is always a risk that between-host selective pressures act against within-host selective pressures.

In the context of microbiota invasions, there is a natural parallel to make with emerging infectious diseases because typically, when a pathogen first emerges, it will face a set of "patches" (i.e. hosts) that are colonised by resident pathogens. In epidemiology, there is a longlasting debate regarding the virulence of emerging infectious diseases. Some argue that these pathogens should be more virulent than resident pathogens, while others argue the opposite. By definition, emerging pathogens are less likely to be adapted to the host than resident pathogens. This does not, however, predict their virulence since maladaptation can lead to high virulence (e.g. due to immunopathological reactions of the host) or low virulence (e.g. rapid clearance by the immune system). However, if stochasticity is taken into account, things change. Indeed, for two pathogens that cause the same number of secondary infections (and should therefore be equally fit a deterministic setting), the less virulent is more likely to emerge because it causes longer infections, which minimises stochasticity (André and Day 2005).

Adopting a meta-community approach to study epidemiology is tempting as hosts can be seen as communities of microbes (see Seabloom et al. 2015 for an interesting attempt). However, beyond the analogy, it is not clear how each field can help each other, especially in terms of virulence. In the earliest meta-population/community models, patch extinction was usually a catastrophic event occurring at a constant rate. This has changed and models now allow this property to depend on patch density or on the status of neighbouring patches (e.g., Eaton et al. 2014). In epidemiology, there has been a longer focus on the idea that virulence is a property that originates from the within-host (i.e., within-patch) dynamics (Sasaki and Iwasa 1991). Both in ecology or epidemiology the challenge raised by including the host microbiota into the picture 
is the same: how to track within-host diversity while allowing for dynamics to take place at a higher level (Sofonea et al. 2015).

\section{Experimental tests of microbiome invasibility}

Culturing microbial communities has played an important role in ecology for several decades (Faust and Raes 2012). However, with more sophisticated cultivation methods and technology advances that allow measurements to be made in unprecedented detail, it is now possible to probe more complex microbial communities and test their stability. Several animal models have been developed in order to study microbe-host interactions and in particular the symbioses between them. Invertebrates (e.g., squid, nematode and Drosophila melanogaster) are best suited for investigating more binary interactions, while vertebrate model systems (e.g., mouse and zebrafish) are best for investigating more complex microbial communities and their interactions with the host (Rader and Guilemin 2013). Indeed, colonisation of germ-free organisms with human microbial communities (e.g., colonisation of zebrafish guts with humanderived anaerobic bacteria Toh et al. 2013) allows for controlled studies of these communities in lab settings. Of particular importance are animal models such as Drosophila or mice that can be manipulated using very precise genetic tools with the aim of defining host-microbe interactions and mechanisms that stabilise communities (reviewed in Lee and Brey 2013 and Goodman 2014).

While there is a long history of infecting animal models with pathogens, these studies have usually aimed for molecular, immunological, genetic, evolutionary or pharmacological insights of host-pathogen interactions and often do not take the presence of a resident microbiota within a given host into consideration. In contrast, emerging microbiome-focused studies have provided a more holistic appreciation of community ecology, in which the focus is how resident microbes can help prevent infection, or how microbial species can be used to restore a dysfunctional ecosystem after perturbation, through assessment of factors such as community stability, resistance and resilience to invasion. The study of fecal ecosystem changes following fecal bacteriotherapy (stool transplants) in human patients with Clostridium difficile infections represents a good example of microbial ecology applied to a medical problem, where the goal is to understand how the presence of certain microbial species can correlate with stability and hence resistance to disease caused by C. difficile (Seekatz et al. 2014; Shahinas et al. 2012; Shankar et al. 2014). In a more targeted approach, a 33-strain defined ecosystem (MET-1) derived from a single healthy human donor was used to treat recurrent $C$. difficile infections in two patients, tracking ecosystem changes with time after administration of this therapeutic ecosystem (Petrof et al. 2013). Both approaches clearly demonstrated a correlation between a 
lack of microbial diversity with the disease state, and a subsequent input of diversity with transplantation that correlated with restitution of the diseased state. The same approach may also be applied for the treatment of other diseases and infections; the 33-strain therapeutic ecosystem used to treat $C$. difficile infection (above) was tested for efficacy against Salmonella enterica infection in a murine model (Martz et al. 2015). S. enterica was chosen in this case because the infection is mediated by bacterial invasion whereas $C$. difficile associated disease is mediated by exotoxin production and the bacterium itself is not invasive. While protection against disease was seen in MET-1 treated animals dosed with $S$. enterica, the mechanism of protection was thought to be a direct result of strengthening of host barrier functions thereby preventing pathogen entry. Thus, microbial ecosystem therapeutics as an approach is likely to have multifactorial effects on host and microbiota, reflective of the complex interactions between these two factors. There remains much to be learned from such studies, integrating microbial communities with host phenotypes and genotypes to determine how both factors interact together during pathogenesis.

An example of a true invasion ecology experiment was conducted by Seedorf et al. (2014) in which they colonised gastrointestinal tracts of germ-free mice with microbial communities obtained from several very different sources (humans, zebrafish, termites, soil and aquatic mats); as such, this can be considered as an invasion island biogeography experiment (Delong 2014). The fact that all communities (albeit from very different origins) were able to colonise the mice and form stable and reproducible populations highlights that there are community assembly rules as well as common properties across communities that allow their members to invade new habitats. In particular, the taxa that were most successful in colonising mice were those with greater capacities to perform certain functions, such as metabolism of host nutrients and bile acids. This study also found that there were repeatable patterns of invading successions, i.e. some taxa invaded first, then declined in abundance, allowing other taxa to follow (for free-living examples of this see Schreiber and Rittenhouse 2004). In addition, they found that Firmicutes species (regardless of origin) were fundamental to the initial colonisation of the gut. These findings support the invasional facilitation hypothesis discussed in Section 2.2, which seeks to explain that the succession of a community is predictable because particular species facilitate invasion by other taxa. These findings also support the premise of keystone species (Paine 1966; Paine 1969), which carry out critical functions required to prime an ecosystem for colonisation and that allow community assembly. Keystone species have been found in several other microbiome studies as well (Allen-Vercoe 2013; Ze et al 2013).

In vitro systems allow for even more control of microbial communities but come with the downside that the contributions of the host to ecosystem parameters can only be measured superficially (e.g., by the addition of host proteins, such as mucins; Allen-Vercoe 2013; McDonald et al. 2013). Culturing microbes from the mammalian gut can be challenging because many are fastidious in their nutritional needs and require cooperation with other species for optimal growth (Allen-Vercoe 2013). Fecal samples offer a convenient starting material from which to culture gut microbial ecosystems, and in vitro bioreactor-based models, with parameters set to mimic conditions of the gut, can be used to support these communities for several weeks at a time, allowing a steady state equilibrium to be achieved that can serve as a 
baseline for experimental perturbations (Figure 4). Interestingly, in human studies, the ecosystem at steady state tends to diverge from the fecal inoculum sample (McDonald et al. 2013; McDonald et al. 2015) and this may reflect the fact that feces contains both autochthonous (resident) and allochthonous (non-resident) microbe representatives of the luminal content. However, a small number of mucosal microbes will be contained within feces and thus attainment of steady state may represent the loss of allochthonous species (which cannot be supported by the ecosystem) as well as the bloom of lower-abundance, mucosally associated microbes. In other words, the in vitro steady-state fecal ecosystem may represent the core microbiota of the human gut, although this remains to be tested.

A

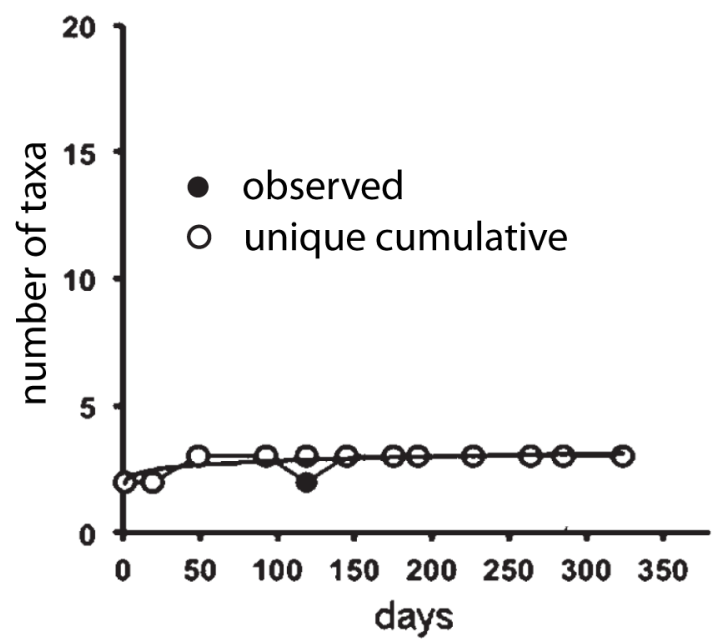

B

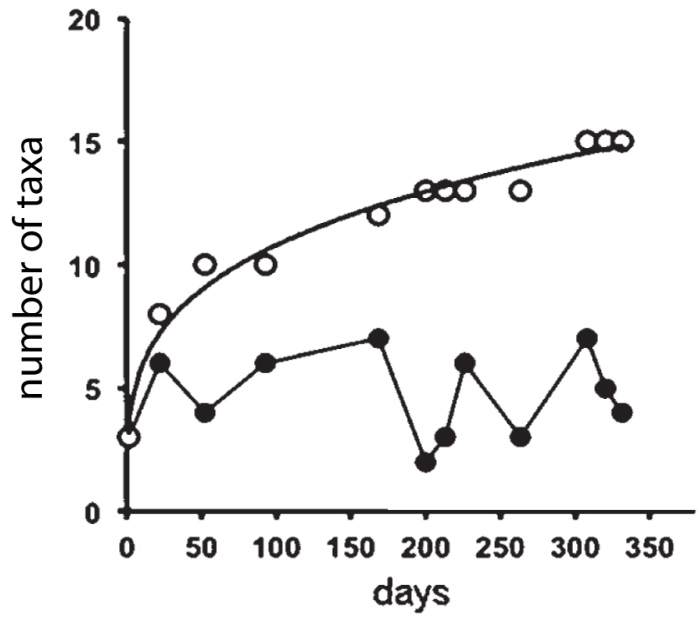

C
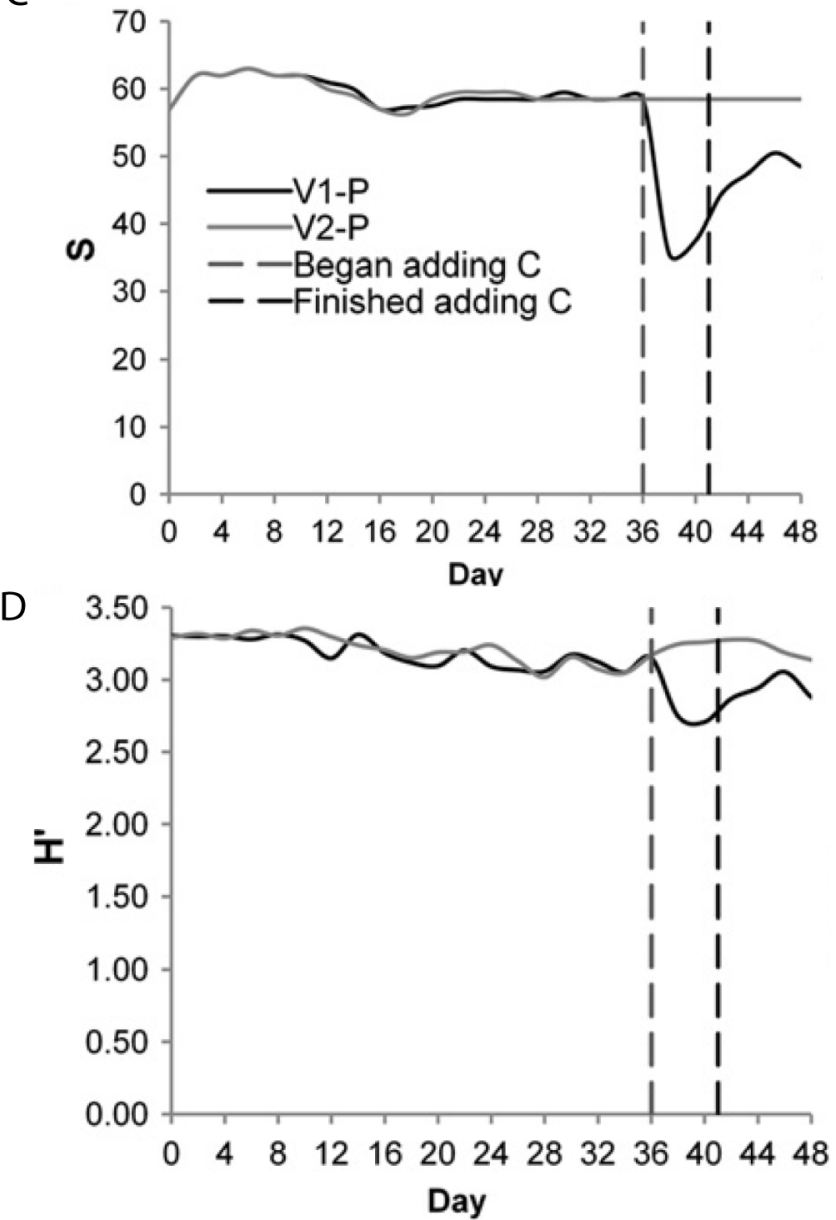

Figure 4 .

Several in vitro systems for the culture of human gut microbiota, as well as oral microbiota using patient- or volunteer-derived samples, have been described and reviewed elsewhere (Edlund et al. 2013; Venema and van den Abbeele 2013). While it is highly valuable to study complex 
communities such as these, there can be problems with reproducibility of these models because inoculum samples can differ if taken at separate time points. The high level of functional redundancy present within a natural microbial ecosystem does, however, lend itself to a partial solution to this problem. Whilst not all of the microbes present within a human-derived ecosystem are easily cultured, it is possible to construct subset communities of these parent ecosystems which retain some functional similarities ( Petrof et al. 2013; Yen et al. 2015). Such ecosystems, although not a complete facsimile of the natural situation, have the distinct advantage of being wholly reproducible, and furthermore, individual taxa can be removed or added precisely in order to determine their effects on the ecosystem. Such insights may then be extrapolated back to the natural ecosystem to help build hypotheses for testing.

Model in vitro ecosystems, both derived from complex or defined communities, are excellent platforms on which to model ecosystem perturbation with only minimal ethical consideration. Such platforms have been extensively used to study host microbiota/pathogen interactions, as well as the effects of dietary substrates, supplements and antibiotics on the microbial ecosystem (Aguirre et al. 2014; Aguirre et al. 2015; Baines et al. 2013; Crowther et al. 2016; Wissenbach et al. 2016). Perturbations can be assessed by measuring phylogenetic, metagenomic, transcriptomic or metabolomic outputs, and, in fact, the platform outperforms animal models in this respect since samples can be obtained from bioreactor vessels very easily and are not altered by absorption or transformation by the host. The platform may be particularly useful for studying microbial succession following invasion because of the controllability of the parameters.

A potentially very fruitful direction for future research would be to combine both in vitro and in vivo experiments with mathematical models incorporating ecological theory in order to help determine the drivers of community stability. Several groups have outlined frameworks for such models (Bucci and Xavier 2014; Moorthy et al. 2015), and a recent and an elegant study by Buffie et al. (2015), successfully combined mathematical modeling with analysis of a mouse model of antibiotic-induced $C$. difficile infection, by specifically determining how microbiota changes associated with antibiotic introduction could be correlated to disease status. The authors were able to extrapolate their findings to human patients and this work indicated that a specific bacterial species, $C$. scindens, greatly contributes to resistance to $C$. difficile infection (Buffie et al. 2015). Approaches such as this may also be applied to other disease states in order to guide rational design of future therapeutic strategies.

Since it is becoming increasingly clear that the diversity of microbial communities significantly contributes to their stability, resilience and resistance (Allen-Vercoe 2013; Lozupone et al. 2012), and that lack of microbial diversity seems to be associated with a growing number of human diseases including obesity, type 2 diabetes and inflammatory bowel diseases (Ridaura et al. 2013; Matsuoka and Kanai 2015; Vrieze et al. 2012), it is important that a fuller understanding of ecosystem dynamics is found, which incorporates the roles of such ecological measures as network interactions and the significance of keystone species. 


\section{Types of invaders}

As laid out in Section 2.2, successful invasion by free-living species into new habitats requires completing 4 steps: transmission out of the home environment, introduction into the new environment, establishment, and spread. WH invaders face these same challenges and they require many traits to complete these stages. Here we discuss invasion strategies by different kinds of invaders.

\subsection{Single and poly-microbial invasions}

Given the tradition of using Koch's postulate approaches to find specific etiological agents for diseases, WH systems have traditionally been studied from the perspective of the invader. Important killers, both historically and presently, such as Mycobacterium tuberculosis, Yersinia pestis (plague), and Streptococcus pneumoniae, have been studied separately from the microbiome they encounter when colonising a new host. Nevertheless, community thinking is becoming more common. In particular, there is active research into how enteric infections, such as Salmonella spp., Clostridium perfringens, Vibrio cholera, Escherichia coli and Enterococcus faecalis, interact with the resident community. Invading strains of bacteria face several challenges when entering a new environment with resident bacteria, such as biofilm adhesion, availability of nutrients (residents can deplete nutrients), antibacterial effectors produced by residents (e.g., bacteriocins, metabolic by-products, etc.) and signalling the immune system (Stecher and Hardt 2011). Due to these bacteria-specific defenses, many invading strains have evolved mechanisms that make them more effective colonisers. For example, $V$. cholerae uses quorum sensing (which is density-dependent chemical signaling allowing perception of conspecific density) to increase the expression of its virulence factors in the presence of conspecifics and to form biofilms when it is at low density, thus giving it an advantage to colonise a gut even when low quantities are ingested (Hammer and Bassler 2003). Indeed, many pathogenic bacteria sense nutrient concentrations in their new environment and then alter their virulence factors in order to better compete with resident taxa (review Rohmer et al. 2011). Finally, from a community perspective, invading bacterial strains must also face natural predators and bacteriophages already present in the WH environment (more on bacteriophages in sections that follow). The degree to which these community members help prevent successful colonisation requires more research.

Historically, a single etiological agent was sought to explain a single disease. However, it is becoming clear that some diseases are caused by a group of microorganisms, i.e. polymicrobial infections. These invaders are often ineffective WH colonisers (or less virulent) unless they are together with other specific taxa. For instance, to test the hypothesis that periodontitis (gum disease) is caused by a disbiotic microbial community, mice were infected with four different bacteria species simultaneously and compared them to mono-infections 
(Chukkapalli et al. 2015). They found that polymicrobial infections were more virulent, formed plaques more readily and altered immunity functioning. Interestingly, it is hypothesised that Porphyromonas gigivalis plays a keystone species role in these polymicrobial infections by actively downregulating immune surveillance, which facilitates the growth of the other species in the biofilms, and these communities then become stable (Hajishengallis and Lamont 2012). In fact, there are many examples of polymicrobial synergy, i.e. the interactive activity of two or more microbes that allows achievement of results otherwise not possible alone (reviewed in Murray et al. 2014). For instance, co-infections in the otitis media by Haemophilus influenza and Moraxella catarrhalis appear to form stronger, more antibiotic-resistant biofilms, than when coinfecting with other bacteria, because of effective quorum sensing between them (Murray et al. 2014).

In another example, multi-species biofilms, grown in vitro beforehand, were placed onto wounds of mice and found that healing was impaired when compared to single-species infections (Dalton et al. 2011). Likewise, in a Drosophila model, Sibley et al. (2008) did several single vs. co-infections of different strains (e.g., of Staphylococcus spp. and Streptococcus spp.) with Pseudomonas aeruginosa and found that in some cases, when infecting together, there is enhanced killing of the host even if some of the strains were not pathogenic on their own. Conversely, they also found that co-infection (i.e., simultaneous infection by two strains or species) can lead to faster clearance (Sibley et al. 2008). Indeed, in a rat model, the same group found that co-infection enhanced inflammation (Duan et al. 2003). Findings like these point to a potential disadvantage of multiple invaders entering at once: they are more conspicuous to the immune system. This could be because they bring more antigen into the site at once or because they cause more local damage; both of which will alert the innate immune response and cause more local inflammation. This reasoning is also used in combination vaccines in which combining vaccines into one shot leads to a stronger and more rapid local immune response. Overall, determining whether invading as a group or alone confers an advantage or disadvantage for the invaders will require more investigation.

Unsurprisingly, the community into which a polymicrobial infection lands matters, since much of the clinical focus of polymicrobial infections comes from studies of patients with some other underlying medical issue, such as cystic fibrosis (a genetic disorder; Rogers et al. 2010), diabetes (Mastropaolo et al. 2005) or immune system disease. The experiments mentioned above by Seedorf et al. (2014) are designed to identify what features of communities may favour establishment of an invader in a new host. They do so by looking at what affects establishment of an invader (i) into a naive or nearly naive habitat (e.g., in newborn infants) or (ii) into an established community. For example, see their stage 4 experiment in which native mice communities invaded stable, non-native communities in other mice, only to quickly destabilise them and take over, thus demonstrating that communities that have longer evolutionary history with the host have an invasion advantage.

As we better characterize healthy and diseased $\mathrm{WH}$ ecosystems, we are discovering more examples of polymicrobial infections. Recently, bacterial vaginosis (BV) has been hypothesized to be initially triggered by the sexual transmission of a structured polymicrobial 
biofilm synthesized by Gardnerella vaginalis, which then subsequently displaces resident lactic acid bacteria (mainly Lactobacillus spp.) from their ecological niche. In fact, the first experiments aiming at testing $G$. vaginalis as the etiologic agent of BV did not fulfill Koch's postulates (Criswell et al. 1969; Gardner and Dukes 1955). Moreover, G. vaginalis can be isolated from healthy genital tracts of adult and adolescent girls (Hickey et al. 2015; Ravel et al. 2013) and healthy genital tracts are free of structured biofilms contrary to genital tracts with BV. It has, thus, been concluded that "there is no BV without G. vaginalis, but G. vaginalis is not BV" and a consensus now exists on the hypothesis that the transmission unit of BV is the structured polymicrobial biofilm (Swidsinski et al. 2014). Interestingly, several different bacterial species co-occur with BV. For instance, more than $90 \%$ of a biofilm identified on vaginal epithelial cells of women with BV was composed of Atopobium vaginae and $G$. vaginalis (Swidsinski et al. 2005). In another study, two of seven key species (Prevotella spp. and $A$. vaginae) were significantly associated with BV and G. vaginalis and Prevotella spp. defined the majority of BV clusters (Datcu et al. 2013). These latter results suggest a specific interaction that may lead to enhanced selective advantage of both bacteria. Such synergistic interactions among the BVassociated bacteria have been observed. On the one hand, the reproduction rate of planktonic (i.e., free living, non-biofim) G. vaginalis and biofilm growth are promoted by the presence of some additional species such as $A$. vaginae, $P$. bivia, F. nucleatum and Mobiluncus mulieris, irrespectively of which other pathogenic species are involved in particular cases of BV, (Machado et al. 2013; Machado and Cerca 2015). On the other hand, some tested BVassociated bacteria had their growth enhanced by the presence of $G$. vaginalis derived biofilms, such as $P$. bivia that was able to increment its concentration within the biofilm, when added to a pre-formed G. vaginalis biofilm (Machado et al. 2013; Pybus and Onderdonk 1997).

As discussed earlier, insights from infectious disease evolution research can help better understand polymicrobial invasions. Studies have shown that if invasion occurs by a group of propagules rather than by a single propagule, this can deeply affect the evolutionary dynamics. In evolutionary biology, this is called budding dispersal (Goodnight 1992). Experimentally, it was shown that this process helps to maintain cooperative behaviour (producing of siderophores) in a bacterial population over generations (Kümmerli et al. 2009). In epidemiology, this is called co-transmission and mathematical modelling predicts that it can affect the evolution of virulence in directions that are counter-intuitive with respect to classic coinfection models (Alizon 2013).

\subsection{Bacteriophages}

As part of microbiome networks, bacteriophages (bacteria-infecting viruses) are major players affecting the function and structure of bacterial communities. Bacteriophages influence both the frequencies of bacterial genotypes (through modification of fitness and/or virulence) and thus the environmental conditions in which bacteria reproduce, through the promotion of some bacterial genotypes providing new structures (e.g., biofilm) or modification of the physiology of the host. Existing data on interactions between bacteriophages and bacteria in human or animal-associated microbiota suggest a major - though under-evaluated - role of 
bacteriophages in the dynamics and evolution of individual bacterial species/genotypes, but also the bacterial community as a whole.

Bacteriophages follow two major cycles of viral reproduction: (i) the lytic cycle consists of infection, replication and lysis of their bacterial hosts (and thus virulent bacteriophages are restricted to horizontal transmission), and (ii) the lysogenic cycle consists of integration of the bacteriophage genome - called a prophage once integrated - into its bacterial host genome. While temperate bacteriophages (those with a lysogenic cycle) are transmitted vertically between bacteria, external factors can trigger induction of the prophage, i.e. expression and encapsidation of the bacteriophage genome, lysis of the bacterial host cell, and the release of viral particles into the environment which can then horizontally infect new susceptible hosts. Temperate phages carry genes that are not required for their own life cycle, but that modify the phenotype (and fitness) of their bacterial host (i.e., transduction of bacterial genes). Bacteriophages are, thus, prime suspects in the adaptation of bacterial pathogens to new eukaryotic hosts and the emergence of new pathogens (Brüssow et al. 2004). Indeed, it has been shown that bacteriophages transduce antibiotic resistance genes (e.g., Enault et al. 2016; Schuch and Fischetti 2006). Moreover, pathogenic strains of Corynebacterium diphtheriae (diphtheria), Salmonella enterica (Salmonellosis), Clostridium botulinum (the leading cause of food poisoning), Streptococcus pyogenes (scarlet fever), Staphylococcus aureus (causing a number of skin and blood diseases), and enterohemorrhagic E. coli (causing hemorrhagic colitis) all harbor toxins with bacteriophage origins. Specifically, some of these toxic proteins are involved in different steps of the bacterial life cycle, such as eukaryotic host immune evasion, intracellular and extracellular colonisation, and proliferation (Boyd 2012; Brüssow et al. 2004).

There are several ways bacteriophages interact with the microbiota (reviewed in De Paepe et al. 2014). One type of interaction, termed community shuffling, may occur when an environmental stressor induces a lysogenic phage to kill its host. While this releases horizontally infectious viral particles that can infect other cells, which in turn may cause further lysis and potential extinction of the bacteriophage and its host. Moreover, the debris resulting from the simultaneous lysis of many bacteria is likely to stimulate a host response. When this happens, the composition of the microbiota will be affected, and opens the opportunity for invasion by new bacterial genotypes or species. Another form of bacteriophage-microbiota interaction, termed 'kill the winner', is one in which bacteriophages will only deplete a resident bacterial population that is above a critical density, meaning that only the dominant members of the ecosystem are directly affected. Conversely, bacteriophages can also indirectly affect microbiome networks by modifying phenotypes and/or fitness of specific bacterial species in the community. It has long been observed that lysogenic bacteriophages allow their host to outcompete their nonlysogenized competitors in environments in which nutrients are scarce, because lysogenic strains, counter-intuitively, have higher metabolic rates, and thus reproduce faster than wild-type non-lysogenized strains (De Paepe et al. 2016; Duerkop et al. 2012; Edlin et al. 1975; Edlin and Bitner 1977). Bacteriophages have also been hypothesized to be involved in bacterial capacity to adapt to adverse environments, for instance through biofilm formation. Indeed, considering that extracellular DNA (eDNA) is a key factor in the biofilm matrix, activation of Streptococcus 
pneumoniae prophage, resulting in host lysis, showed that localized release of eDNA favours biofilm formation by the remaining bacterial population (Carrolo et al. 2010).

Another interaction is the use of bacteriophages by bacteria as "natural" biological weapons that they deploy to 'kill the relatives' that do not harbor the same prophages (i.e., identifying which resident relatives are not part of the same immunity-group; Brown et al. 2006). This applies particularly in cases in which new bacterial genotypes invade new ecological niches and use bacteriophages as allelopathic agents that will replicate into, and lyse, their targets. In addition, owing to the fact that some bacteriophages present a large host-range, such as those able to infect bacteria belonging to different families (Meaden and Koskella 2013), this interaction probably affects other susceptible bacterial species. However, this interaction can only explain short-term invasions. In fact, taking the example of entero-hemorrhagic $E$. coli, it has also been shown in vivo in the murine gastrointestinal tract that resident susceptible $E$. coli were lysogenysed by Shiga toxin-converting prophages present in the invading entero-hemorrhagic E. coli (Acheson et al. 1998). Thus, after horizontal transmission, lysogenic phages may establish themselves in the resident bacterial hosts and then confer immunity to bacteriophages. This property would then confer a disadvantage to the invading lysogenic bacteria. Moreover, the presence of new resident lysogenic genotypes will even accelerate as the bacteriophage population size grows because lysogeny rate has been shown to increase with increases in multiplicity of infection (Gama et al. 2013).

To date, no human disease has been strictly related to virulent bacteriophage invasion and depletion of bacterial communities. Still, several dysbioses have been hypothesized to have a direct bacteriophage origin such as Crohn's disease and ulcerative colitis, or bacterial vaginosis (Kiliç et al. 2001; Norman et al. 2015; Turovskiy et al. 2011). For instance, Crohn's disease and ulcerative colitis have been shown to be associated with a significant expansion of bacteriophages belonging to the Caudovirales family and a decrease in bacterial diversity (Norman et al. 2015). Moreover, bacteriophages were mostly virulent (T4 and T4-like) in individuals suffering from diarrhea, whereas they were mostly temperate in samples from healthy controls (Chibani-chennou et al. 2004; Furuse et al. 1983). As new technological tools continue to increase the precision with which we understand spatial and temporal compositions of bacteriophage-bacterial communities, it will help inform interpretations of clinical and biological data that will, in turn, allow the application of better and potentially new therapies such as bacteriophage therapy (see section 7.1).

\section{Box. 2 - Unique features of other kinds of invaders}

[[ Box 2 here ]] 


\section{Actively acquired invasions}

As in free-living systems, humans attempt to engineer the ecological communities inside their bodies in many ways. Here we discuss examples of biological invaders that we use either to prevent disease (i.e., prophylactics) or as treatments. Prophylactic practices are often used to prevent other invasions, which can be done directly by adding a new resident to the community (e.g., adding a bacteriophage to guard against an unwanted infection) or indirectly by training the immune response to identify a pathogenic invader (e.g., using live vaccines to prime the adaptive immune response). These $\mathrm{WH}$ biological control agents can themselves be single or multiple agents administered into human bodies. Consequently the way they subsequently interact with the WH system can be quite different (e.g., a single bacteriophage can cause predator-prey dynamics while cocktails of probiotics composed of several antagonist microorganisms such as commensal bacteria and bacteriophages can engage in many forms of interactions with resident community members). From a network perspective, these therapies can remove single nodes or multiple nodes of the resident community or even replace the entire system. Therefore, achieving success with these control methods challenges us to both understand $\mathrm{WH}$ invasions better but also to see where our gaps in ecology and evolution are in general.

\subsection{Bacteriophage therapies}

Pioneered by the work of Twort and Felix d'Hérelle in the early part of the 20th century, bacteriophage therapy has a long history of study and application, particularly in Europe and the states of the former Soviet Union. Despite early enthusiasm, it was displaced by the advent of cheap, effective antibiotics in Western medicine in the 1940s. The promise of bacteriophage therapy as a weapon against bacterial infection is three-fold (comprehensively reviewed in Keen 2012; Loc-Carrillo and Abedon 2011). First, in contrast to broad-spectrum antibiotics (Looft and Allen 2012), targeted specificity for a single pathogen should have minimal impact on the microbiome community (aside from control of the pathogenic bacterium being targeted), and very few direct negative effects on the infected host. The second benefit is that bacteriophage will amplify itself as it destroys its target bacterium, reducing the need for repeated administration to the patient. The third intended benefit is that bacteriophage has the ability to adapt in response to the evolution of resistance in the targeted bacteria. It is thus touted as an evolution-proof alternative to the application of static chemical treatments. Though this may not always be the case (see Meaden and Koskella 2013 for examples), bacterial resistance to bacteriophage is generally expected to occur through the loss of the receptors to which they bind on the bacterium's cell surface, which are often the very virulence factors causing the disease (Inal 2003). While this may make the bacteriophage itself less able to propagate and spread, the goal of treating the disease would nonetheless be achieved through attenuation of pathogenicity. Among the few empirical examples to test this, it was demonstrated that bacteriophages selected to target Yersinia pestis virulence factors induced bacteriophage- 
resistant mutations which largely attenuated virulence, while the remaining few had no effect on host mortality (Filippov et al. 2011). Despite the ability of bacteria to become resistant to therapeutic bacteriophages, in vitro experimental coevolution can increase the efficiency of bacteriophage therapy to limit the evolution of resistance of bacteria targeted by the bacteriophages (Friman et al. 2016). These results thus call for a possible individual adjustment of therapeutic bacteriophages according to the bacterial genotypes infecting each patient. Moreover, such studies exemplify the fact that bacteriophage therapy is evolution-proof at a short and manageable scale contrary to other therapies (e.g., antibiotic or antimicrobial peptides). Nevertheless, multi-bacteriophage cocktails can be used to reduce the chances of resistance (Torres-Barcelo and Hochberg 2016) and combination with antibiotic treatments can also have synergistic impacts which improve treatment efficacy (Santos et al. 2009). Bacteriophage-antibiotic synergy is probably the result of several processes, both mechanistic and populational. One mechanism underlying this synergy could be the employment by bacteriophages of efflux pumps as receptors: if this were to be the case, bacterial resistance to bacteriophage would lead to decreased pump activity and consequently increased sensitivity to antibiotics (Chaturongakul and Ounjai 2014). Synergy also arises due to the fact that bacteriophages decrease the bacterial population size and thus increase the efficiency of the chemical molecules targeting the remaining bacteria. As a concomitant effect, natural selection by bacteriophages significantly constrains the evolution of antibiotic resistance as it decreases the rate of appearance of bacteria resistant to antibiotics while also reducing the minimum concentration level of antibiotic necessary to inhibit the growth of the resistant bacteria (TorresBarceló et al. 2016). In addition to the interest in bacteriophage therapy for overcoming established multi-drug resistant infections, multi-phage cocktails have also been shown to act as an effective prophylactics (e.g., in preventing dysentery in children Sulakvelidze 2011).

While the victories of bacteriophage therapy are encouraging, there are some aspects that must be considered when designing a bacteriophage that will be able to invade and reach its target. First, like any foreign invader of the body, bacteriophage elicits both innate and adaptive immune responses upon entry into the host (Kaur et al. 2012). Bacteriophages must be selected or modified to escape innate immune mechanisms, and to control the pathogen before neutralizing anti-bacteriophage antibodies start to be produced. Furthermore, bacterial cell lysis by the bacteriophage can induce further responses from the immune system. For these reasons, cross-reactive adaptive immunity must be considered if further treatment against the same bacterium is required. Second, effective bacteriophage discovery relies heavily on understanding the pharmacokinetics, absorption and distribution, which respectively determine the ability of the phage to disseminate from the site of administration to the site of action and to enter the target bacterium (Abedon 2014). Third, temperate phages - which, as discussed earlier, can result in transduction of antibiotic resistant genes or bacterial virulence factors should clearly not be used, despite any benefits they may offer (Gama et al. 2013). Although bacteriophages in natural systems may run the risk of destabilising microbial communities and "healthy" function, targeting of bacteriophage to a single pathogenic bacterium causing dysbiosis in a diseased patient should lessen the likelihood of disruption to the host's natural microbial community. While bacteriophage therapy seems to yield promising results, the 
population and evolutionary dynamics have yet to be thoroughly explored in the context of the human microbiome (Levin and Bull 2004).

\subsection{Probiotics and food-borne invaders}

Humans regularly ingest bacteria in food, especially from dairy products and fermented foods (Lang et al. 2014). The idea that these are "good" bacteria is quite old. However, research into the human microbiome is revealing how these groups of potentially helpful invaders can affect our resident communities. Metagenomic analyses are being used to identify the various bacteria and even viruses in our foods (e.g., in Kimchi a fermented traditional Korean food Jung et al. 2011). Food-borne bacteria can interact with the resident microbiota in various ways, both directly and indirectly (e.g., supplying metabolites or stimulating mucin production, which would then affect resident mucosa-associated bacteria), and, thus, have effects on their fitness (Derrien and van Hylckama Vlieg 2015). In fact, some argue that fermented foods could serve as experimentally tractable systems to study microbial community assembly, dynamics and invasibility (Wolfe and Dutton 2015).

Probiotics are bacteria used to treat or prevent health disorders, and thus they must be nonpathogenic. There are many ways to introduce probiotics, such as food (e.g. yogurts with Bifidobacteria), capsules filled with bacterial cultures (e.g., Lactobacillus acidophilus pills) and creams or suppositories (e.g., vaginal suppositories with Lactobacilli). Some desired effects are: secretion of inhibitory or beneficial substances, inhibition of growth of pathogens and positive interactions with the immune system (Macfarlane and Macfarlane 2013). Probiotics are often marketed as helpful for maintaining a healthy, "balanced" microbiome. However, it is far from understood what constitutes a healthy and stable microbiome. This is particularly challenging because there are many "healthy" microbiomes, i.e. various stable communities with different taxonomic compositions, so how to define a healthy microbiome system is still under debate. Consequently, using a more ecological function approach, probiotics in the future may be designed for bolstering functional attributes of the resident community (Lemon et al. 2012).

The aim of treating or preventing obesity (i.e., improving patients' lipid profiles) has led to many studies into potential probiotic treatments. The main premise is that the ingestion of certain bacterial species (e.g. Lactobacillus fermentus, certain L. plantarum strains, L. reuteri) may lead to particular activities of these species, such as interactions with cholesterol, thereby reducing circulating levels of cholesterol in blood. There are several proposed mechanisms by which candidate bacterial species may produce this desired effect. For example, probiotic strains can bind cholesterol directly, incorporate it into their cell membranes, convert cholesterol into coprostanol which then gets directly excreted, induce enzymatic deconjugation of bile acids, etc. (reviewed in Ooi and Liong 2010). However, the results of in vivo and human trials have been mixed. It could be that the interactions with the resident community are contributing to creating this bag of mixed results. For instance, probiotics that induce bile salt deconjugation subsequently liberate amino acids that can be used as sources of carbon and nitrogen by the invading species (Begley et al. 2006), but also by other species around it. Producing nutrient 
sources to local species should influence not just the likelihood of the probiotic species to establish in the community, but also how cholesterol levels are affected overall.

The successful establishment of an invader is, of course, fundamental to the success of probiotic treatments. For example, a cholesterol-reducing effect in mice, but long-term establishment of the probiotic bacteria was not obtained ( $\mathrm{Li}$ et al. 1998). Indeed, a common failing of probiotic treatments and trials is that the probiotic bacteria are not able to colonise, i.e. establish in the resident community, or do so only transiently. In ecological terms, the invaders are not able to fully go through all steps in the invasion process (particularly, establishment and spread). Human gut communities are often found to be persistent when faced with food-borne or probiotic treatments. For example, Zhang et al. (2016) found that the structure of the gut community, and not its diversity, affected how quickly an invasive, food-borne bacteria was cleared from the system. Studies interested in this topic often focus on finding which specific bacterial phyla were correlated with persistent communities or with the resilience of the community. Perhaps, however, a network approach, such as inferring the pre-invasion resident network and seeing how its properties change (e.g. types of interactions or other network statistics), would help improve inferring what properties of the communities make them permissive. In addition, the insights from these studies will help uncover what strategies $\mathrm{WH}$ invaders need (and what trade-offs shape them) in order to successfully establish. Hence, studies in probiotics and food-borne invasions could help both advance WH community studies and increase the efficacy of these medical therapies.

\subsection{Other therapies}

Fecal bacteriotherapy follows the principle of probiotic use, but instead of a single or limited number of species, fecal bacteriotherapy aims to incorporate an entire healthy ecosystem into a sick ecosystem, either to integrate with, or to displace, the sick ecosystem. The process itself is fairly primitive; in general, a healthy donor is sought and then screened for the presence of pathogens in their stool, and provided these are not present, then a fecal sample is obtained from this donor, homogenized into a slurry with water or saline solution, and instilled directly into a patient (Allen-Vercoe et al. 2012). The approach has become fairly widespread in the treatment of recurrent $C$. difficile infection, and is associated with a very high cure rate of over $90 \%$ (van Nood et al. 2013). However, the procedure is not without risk, particularly from the transfer of as-yet unknown pathogens from donor to patient, as well as the risk of 'unknown unknowns' such as long-term complications of gut microbiota modulation which have yet to be measured (Allen-Vercoe et al. 2012; Allen-Vercoe and Petrof 2013). Despite this, the potential of the therapy for the treatment of other diseases with gut microbiota disturbance as a key feature, such as ulcerative colitis and type 2 diabetes, is currently being explored (Vrieze et al. 2012). The approach is also subject to improvement. A recent study, Li et al. (2016) examined the fate of applied and autochthonous communities for up to 3 months following fecal microbiota transplantation (FMT) for the treatment of metabolic syndrome and found that FMT-supplied strains of the same species as the recipient's microbiota tended to integrate themselves more readily than new species into the community after treatment. However, the patterns of microbial 
replacement/colonisation in each recipient were found to be quite distinct from each other, suggesting a large host-driven effect on gut microbiota colonisation. Several groups have been developing standardized or defined microbial ecosystems as treatment modalities (instead of feces, which is inherently variable; Khanna et al. 2016; Petrof et al. 2013; Ray and Jones 2016). Such standardized therapies will contribute to our understanding of how microbial ecosystem invasions affect health, and will shape this therapeutic approach further in the future.

In the face of globally rising antibiotic resistance, predatory bacteria are being considered as potential alternatives and have shown effectiveness against biofilms, which are particularly tough to break up even by antibiotics (Allen et al. 2014). Ecologically, this strategy is one that adds a trophic level and strong new links of predator-prey interactions. Thus, it is not surprising that these predatory bacteria therapies have the potential to effectively destabilise a pathogenic community. Nevertheless, as mentioned above (in bacteriophage therapies), unwanted effects on the resident bacterial communities of the host are a real danger and considerable research is needed to investigate their safety. Here, using an ecological network approach would help better understand how these predatory bacteria interact with their new community.

Other examples of human-induced biological invaders that are discussed less in the microbiome literature are live vaccines. Live vaccines are vaccines where the pathogenic microorganism (viruses or bacteria) are attenuated and these new strains with lost functions are used to prime immune responses to prevent later challenge infections by more pathogenic strains. Attenuated live vaccines are some of the most successful infection control in history (just one example: eradicating smallpox), and arguably some of the most successful biological control strategies across all ecosystems. These vaccines show the central importance the immune response plays in protecting the host (and its residents) from invasions. This is highlighted by the fact that it is recommended not to vaccinate immunosuppressed hosts with live vaccines since, in these hosts, the vaccine-strains can cause infection and disease (Kew et al. 2005; Shearer et al. 2014).

Generally, any live vaccine strain has the potential to interact with the resident community. A viral example is that the oral poliovirus vaccine strains can interact with other resident, distantly related enteroviruses, recombine and regain their virulence (Riquet et al. 2008), causing disease and potentially spreading to other hosts. This example highlights the importance of the human virome, which is less understood than the microbiome. In spite of these potential resident-invader interactions, the fact that they are very rare speaks volumes to the efficacy of the immune system (i.e., stronger predation than in free-living systems). However, as we develop new live vaccines, particularly against bacterial pathogens (Girard et al. 2006), we need to be mindful of the resident community (Detmer and Glenting 2006). Of particular worry are gene transfers and unexpected bacteria-bacteria interactions. Interestingly, vaccine designers are experimenting with answering similar ecological questions as discussed in this paper. Namely, what properties of the invader, the immune system or of the system itself make an invasion event unsuccessful? Understanding these mechanisms, which include ecological considerations, will be important for future successes of novel vaccines. 
Likewise, live therapies (probiotics, transplants, predator introductions, etc.) can have long-term, potentially unwanted effects. Indeed our history of biological control in free-living ecological systems offers up many examples. Some issues to keep in mind are: unintended effects on the non-targeted species can be both direct and indirect, introduced invaders can disperse from the target site, relatives of the target species are likely to be affected, and underlying environmental conditions affect the impact of the invader (Louda et al. 2003). A currently popular technique is 'inundative' biological control, whereby large numbers of the invader are introduced at once with the intention to have a high dose effect and not necessarily be a long-term pest management strategy. It is not clear that this control strategy is able to avoid the long-term issues that classic biological control have on non-target species (Van Lenteren et al. 2003). However, dose effects should also play a key role in the success of invasions and thus live therapies. Quorum sensing is one important reason why this is the case (see polymicrobial infections in 6.1). Therefore, applying this inundative approach may increase the chances of success, but maintain the risk of unwanted effects. Finally, a potential, and particularly unwanted, non-target effect of live therapies is evolutionary effects, such as the evolution of virulence (Ford and King 2016). With the aim of introducing stronger competitors, therapies may inadvertently select for higher virulence in the target pathogen, specifically by increasing the weapons it uses against the new residents (Ford and King 2016; Vale et al. 2016). However, it has been suggested that if the therapy is sufficiently aggressive, i.e. high pathogen killing, then this evolutionary response could be avoided (McNally et al. 2015). Concomitantly, the new residents introduced by the therapy themselves can evolve novel weapons to increase their competitive ability, which is an idea coming from free-living invasion biology, namely the novel weapons hypothesis (reviewed in Callaway and Ridenour 2004). Understanding potential long-term evolutionary outcomes will play an important role in ensuring efficacy of these live human-induced invasion treatments.

\subsection{A note on non-living perturbations}

Perturbations not due to biological invaders play a very important role in shaping $\mathrm{WH}$ communities. Abiotic disturbances can be seen through the analogy of environmental shifts such as changes in nutrient gradients, or where the concept of catastrophic regime shifts applies, i.e. when a disturbance is sufficiently large that the community shifts to an alternative stable state (Relman 2012; Scheffer et al. 2001; Scheffer and Carpenter 2003;). Indeed, the concept of alternative stable states (or multistability; see Section 3.1) is one that has been embraced by microbiome researchers. An example an abiotic driver of changing states is a study of small bowl transplant patients that found that the oxygen brought in by an ileostomy changed the gut community away from a strictly anaerobe dominated community, but then it went back to the original resident community composition after the ileostomy was closed (Hartman et al. 2009). Often abiotic perturbations can precede biological invasions and change the properties of the resident community to be more susceptible to invasions. The most common form of abiotic disturbance of the microbiome is antibiotic use. Antibiotics have the potential to directly affect the stability and resilience of the microbiome to biological invasions. Antibiotic administration represents a strong perturbation that inflicts heavy collateral damage to 
an ecosystem (see Figure 4 C and D; Antonopoulos et al. 2009; Heinsen et al. 2015; Jakobsson et al. 2010; Looft and Allen 2012; Nobel et al. 2015; Robinson and Young 2010). It has been repeatedly shown that antibiotic use negatively affects microbial community diversity and hence resilience, potentially allowing microbiome communities to become more susceptible to invasions by pathogens such as C.difficile (Allen-Vercoe 2013).

The non-living components of diet, e.g. abiotic or biotic compounds that are ingested, are further key modulators of the gut microbiome. This is especially the case because different dietary substrates stimulate the growth of different gut microbes; for example, prebiotic substrates inulin and arabinoxylan have been shown to stimulate the growth of different groups of bifidobacteria (Van den Abbeele et al. 2013). Diet also greatly affects not only the abundance of microbial community members, but also the metabolic output of an ecosystem. An elegant example of this was demonstrated by switching the diets of healthy African American and rural South African men for a two-week period, whereby the Americans, whose diets shifted from high fat, low-fibre to low fat, high fibre, experienced a reduction in detrimental fecal metabolites such as choline (a precursor of the atherogenic metabolite trimethylamine $\mathrm{N}$-oxide), whereas the South Africans on the reciprocal diet experienced an increase in detrimental metabolites as well as increased markers of intestinal inflammation (O'Keefe et al. 2015). See Figure 2B for an example of a community dynamic response to changes in diet. Finally, the western diet is also rich in food additives such as artificial sweeteners and emulsifiers, each of which have been clearly demonstrated to cause disturbances in the gut microbiota in animal models (Chassaing et al. 2015; Suez et al. 2014).

Drugs other than antibiotics also represent a further modulator of the gut microbiota, although the impact of many drugs on microbial ecosystems have yet to be evaluated. There is, however, clear evidence of the modulatory effects of particular drugs such as metformin on the gut microbiota (Cabreiro 2016 and reviewed by Li et al. 2016). Overall, it is encouraging to see the application of ecological concepts in these applied research fields, and more interaction between ecologists and microbiome researchers promises to be fruitful.

\section{Perspectives and Conclusions}

We have assembled work from various disciplines with the intention to understand biological invasions of within-host systems from an ecological perspective. The emerging picture is a fastmoving field with ambitious goals and substantial challenges that need to be met in the near future. The host-associated microbiome communities measured so far vary widely in their species richness, have low connectance, and high assortativity. Community dynamics can appear to exhibit alternative stable states and diversity does not seem to be strictly tied to stability (Table 1). 
One of the benefits of working under an evolution and ecology framework is that it allows for finding patterns and general principles that might otherwise be seen as specifics of a particular infection or medical treatment. The theoretical framework of networks and community dynamics is naturally suited for this pursuit. It can help formulate hypotheses in mathematical terms, explain high-dimensional features of large, complex datasets, and incorporate data obtained from various techniques.

Nevertheless, given that network inference and analysis methods are still under development, it is important to be mindful of what are the underlying assumptions and that they best represent our most up-to-date ecological understanding. There is a risk of over-simplifying ecological concepts, which can then lead to incorrectly inferred networks or erroneous predictions. Indeed, not all past examples of research communities embracing the conceptually attractive autonomously regulated complex network have turned out well (Eichmann 2008). In a topic with so much complexity and with experimental techniques still under development, we would be wise then to keep hypotheses grounded in thoroughly vetted experimental conclusions obtained from techniques validated in multiple ways. This is certainly a challenge worth meeting because the payoff is substantial. We are living in a time when some of the most exciting community ecology experiments are happening inside labs trying to cure diarrheal infections. While microbiome research is a relatively new field, this excitement has rapidly attracted researchers that have brought with them a constellation of state-of-the-art approaches that together promise to build a vibrant understanding of this new, and fascinating biological domain. Greater communication across fields will facilitate the successful integration of this constellation and help accelerate our knowledge of not only $\mathrm{WH}$ invasions, but biological invasions in general.

\section{Acknowledgments}

S. A. and CL. M. would like to acknowledge funding from the European Research Council (ERC) under the European Union's Horizon 2020 research and innovation programme (grant agreement No 648963). We would also like to thank our reviewers for improving this review.

\section{Table and Figure Captions}

Table1.

Comparison between microbiome ecological networks and free-living ecological networks, with suggested implications for the stability and invasibility of the microbiome community. 


\section{Figure 1.}

Different body-site communities have heterogeneous network properties and diversity. (A) Community interaction networks from different human body sites. Red nodes are sourcespecific nutrients, while orange and yellow nodes are microbial genera. Links are either consumer-resource interactions or facilitative interactions. Image modified with permission from chapter 2 of (Ritchie 2011). (B) Network properties of 11 human habitats. Table from (Jordán et al. 2015). Nodes are OTUs; density is the number of links divided by the maximum number of links; APL is average path length; and transitivity is the clustering coefficient. (C,D) Heterogeneous diversity across body-site specific microbiomes. (C) Rank abundance curves of bacterial genera from 22 human habitats. The female reproductive habitats (light blue curves) have few genera dominating their communities, while oral habitats (red curves) have fewer dominant genera. (D) Shannon diversity boxplots of the same 22 human habitats. According to this index, the abundances of oral communities (left) are the most uniform, while those of vaginal communities (right) are the least uniform. C and D used with permission from (Zhou et al. 2013).

\section{Figure 2.}

Inferring microbiome interaction networks using community dynamics. The growth, interaction, and perturbation parameters of community dynamics of the form of Eq.1 (see Section 3) and shown in (A) are inferred from time series of expression of all species, two of which are shown in (B), measured over a window within which a step-like environmental change is made (here high- to low-fibre, and back again). (C) A similar procedure is applied to a different network observed over a window in which Clostridium difficile (green circle) is introduced. Blue arrows denote positive interaction strengths, while red denotes negative ones. Thickness denotes interaction strength. The authors find that, of all nodes, three (Bacillus fragilis, Clostridium hiranonis, and Roseburia hominis, thick circles) are necessary and sufficient to effectively repel the invasion. In this network representation, a particular pathway (green dashes) involving these three can indeed inhibit $C$. difficile. Modified images from (Bucci et al. 2016).

\section{Figure 3.}

Interaction networks can be decomposed into ecological modules. Left: a schematic caricature of a sinus microbiome interaction network. A diamond and single chain module are highlighted by the dashed ovals. Right: A set of typical modules, labeled with their name and showing the resource, $\mathrm{R}$, the consumers, $\mathrm{C}$, and the predator, $\mathrm{P}$ (modified with permission from (Murall et al. 2012).

\section{Figure 4.}

Time series of microbiome communities. $(A, B)$ Community of lung microbial communities of two cystic fibrosis patients over a period of one year, measured using terminal restriction fragment length polymorphism. Diversity, or the number of taxa, is shown as filled circles, while the unique cumulative diversity, or the number of unique taxa seen up to that time, is shown as 
open circles. The community in A shows little turnover and appears to persist in time, while that in B shows high turnover with the numbers of taxa fluctuating in time. Modified from (Stressmann et al. 2012). (C,D) Response of an in vitro community to antibiotic treatment. Denaturing gradient gel electrophoresis analysis of the planktonic communities from two chemostat vessels (V1-P, black line and V2-P, grey line) modeling the human distal gut prior to, during, and following a clindamycin treatment period (demarked by vertical dashed lines). From the number of bands taken as species, $S$ (shown in $C$ ), and their relative intensities taken as relative abundances, the adjusted Shannon diversity index, $\mathrm{H}^{\prime}$ was calculated and shown in $\mathrm{D}$. Figure from (McDonald et al. 2015).

\section{Boxes and Tables}

\section{Box 1.}

\section{Does relatedness help or hurt a species' ability to invade?}

Ecological studies have shown us that there can be both positive and negative consequences to sexual reproduction of invaders with taxa in the new environment (see Section 2), and similarly, functional or genetic relatedness between $\mathrm{WH}$ invaders and the resident microbiome can affect invasibility success, establishment and spread. Cross-immunity (or cross-reactivity) is a major evolutionary force that affects pathogen diversity (i.e. it drives viruses and microbes to be as distinct as possible from one another in order to avoid immunity detection, memory recognition and clearance). Mechanisms to escape cross-reactive immune responses include recombination (i.e., microbe sex), rapid generation times and substitution rates, and antigenic shedding. Paradoxically, interactions with related resident taxa can both help (e.g. provide beneficial genes conferring higher fitness, via recombination or gene transfer) or hinder (e.g., introducing genes conferring lower fitness) the invader. Finally, related resident taxa can both prime the environment to help invaders but can also be competitive within a shared 'niche', making it harder to invade (i.e., Darwin's naturalisation hypothesis). Whether relatedness of the invader to resident taxa indicates a permissive environment (termed the pre-adaptation hypothesis) or a more challenging battle with these native competitors is the subject of much debate. A recent meta-analysis across mostly plant and animal invasion studies found that the answer to this "conundrum" appears to be dependent on the scale at which it is measured, but the patterns are fairly consistent across wide variation in taxa and ecosystems (Ma et al. 2016). Thus, determining whether relatedness can help or hurt invasion success in the microbiome will require similar studies and meta-analysis across various kinds of invaders (viruses, microbes, etc.) in order to find out whether these apparently global patterns also 
apply to WH microbial systems.

\section{Box 2.}

\section{Viruses (other than bacteriophages)}

While viral infections are usually studied on their own without consideration for the microbial communities into which they land, there are examples of how resident microbes can protect the host from viral infections. For instance, Wolbachia, a gram-negative alpha-proteobacteria that infects insects has been shown to protect Drosophila or mosquitos from RNA viruses (e.g., Dengue or Chikungunya virus). It uses mechanisms such as competition for cholesterol and regulating host gene expression, and thus inhibits virus replication (Hamilton and Perlman 2013).

Some viruses, like human immunodeficiency virus (HIV), can have catastrophic effects on the entire WH ecosystem because they target the cells of the immune system, allowing themselves to be captured only to then destroy the cell (Carrington et al. 1999). By disabling the immune system, the virus is free to proliferate, as are all other pathogens (and the microbiome) that had previously been regulated by the immune system. This manipulation of the host immune defenses can also be specific, such as in antibody-dependent enhancement of dengue virus. Here, the initial infection provokes a normal antibody response targeting the virus. However, upon re-infection with a sufficiently heterologous dengue virus, those antibodies still bind the virus, but trigger enlargement of the infected cell rather than neutralization, allowing the virus to replicate unchecked and causing severe disease (Flipse et al. 2013).

With increased metagenomic approaches and microbiome research, we hope that human studies of viral infections will now investigate more direct interactions between the microbiota and viruses. These interactions may be playing a bigger role than is currently believed in preventing invasions, and affecting the immune system's efficacy, infection virulence or duration of infection.

\section{Macroparasites}

Macroparasites, such as intestinal worms, are likely to be involved in many trophic interactions in the gut, though studies are lacking. Helminths and the microbiome do appear to interact both directly and indirectly, and especially through the immune system (Zaiss and Harris 2016). Theories of the association between helminth infection and anorexia are numerous and largely still unsubstantiated, but include the possibility of a behavioral response of the host to change amount or selectivity of food intake in order to enhance immune response to the infection (Kyriazakis et al. 1998). If this is the case, it is also likely to have impacts on the composition of host intestinal flora.

Invasive non-typhoidal Salmonella enterica is strongly associated to populations with high malaria transmission, and in addition to immunological potential mechanisms, malaria's sequestration of red blood cells in the intestine could free iron, which promotes Salmonella growth and the translocation of the bacteria through the gut barrier (Biggs et al. 2014).

Co-infections of macroparasite and microparasites affect the immune response in a unique way, whereby they trigger different, yet competing, immune response pathways (Th2 and Th1 respectively). This can lead to longer durations of infection or immunity escape (reviewed in Maizels and Yazdanbakhsh 2003). Whether and how this Th1/Th2 switch affects the dynamics of the WH system (resident microbiota with the immune response) and the resulting epidemiology is an interesting avenue to consider (e.g., Ezenwa and Jolles 2015). For the microbiome in 
particular, both responses may be involved in WH interactions, because intracellular bacteria trigger the Th1 response, whereas those that proliferate outside of the cell trigger the competing Th2 responses.

\section{Yeast and Fungi}

Fungal species can form biofilms along with resident species and as such are often implicated in polymicrobial biofilms and their associated diseases. A very common example of a pathogenic yeast is Candida albicans which can have both positive or negative interactions with resident bacterial species; for example it forms a scaffold base on which Staphylococcus aureus builds its biofilm (which it does not readily do on its own) and it can facilitate the growth of Staphylococcus epidermidis, yet Candida interacts antagonistically with Lactobacillus (Harriott and Noverr 2011) 


\begin{tabular}{|c|c|c|c|}
\hline & & \multirow[b]{2}{*}{ Implications for invasibility } \\
\hline Property & microbiome networks: & free-living ecological networks: & \\
\hline $\begin{array}{l}\text { Network size } \\
\text { (total number nodes, i.e. } \\
\text { diversity) }\end{array}$ & $\begin{array}{l}\text { Dysbiotic communities (which are usually lower in diversity than } \\
\text { healthy communities) are more susceptible to invasions (e.g. in } \\
\text { murine experiments artificially low diversity communities are more } \\
\text { susceptible to infection by Salmonella enterica, but that protection } \\
\text { can be restored by raising these mice with other mice having a diverse } \\
\text { microbiota (Stecher et al. 2010). } \\
\text { Conversely, the vaginal microbiome is significantly less diverse than } \\
\text { other communities (such as the gut) and yet, it is very stable and } \\
\text { resistant to invasions (Jordán et al. 2015). }\end{array}$ & $\begin{array}{l}\text { The positive relationship between high diversity and } \\
\text { resistance to invasion is found frequently in theoretical } \\
\text { literature, leading to the "diversity resistance hypothesis"; } \\
\text { suggesting that highly diverse systems have more } \\
\text { competitive links and can thus resist invasion more readily. } \\
\text { However the mechanisms are not well understood (Stecher } \\
\text { et al. 2010) and this is still not experimentally well founded } \\
\text { (Levine 1999). For example, positive interactions may alter } \\
\text { this theoretical hypothesis. }\end{array}$ & $\begin{array}{l}\text { Diversity alone probably does not determine the susceptibility } \\
\text { to invasion; other features are important and underlie the } \\
\text { mechanisms behind the diversity-stability relationship (see } \\
\text { diversity-stability debate, review (McCann 2000)) }\end{array}$ \\
\hline $\begin{array}{l}\text { Motifs } \\
\text { (and ecological modules) }\end{array}$ & $\begin{array}{l}\text { Motifs found: } \\
4 \text { motifs in healthy patients vs. } 3 \text { motifs in alcoholics, and some motifs } \\
\text { are more abundant in healthy than in alcoholics (Naqvi et al. 2010). } \\
\text { Network inference of gut data found the network was a composite of } \\
\text { several types: scale-free, band-like and clusters of subnetworks (Kurtz } \\
\text { et al. 2015) }\end{array}$ & $\begin{array}{l}\text { Ecological systems have been found to contain several over- } \\
\text { represented motifs: food chain, omnivory, generalist and } \\
\text { apparent competition motif (Milo et al. 2002). } \\
\text { Energy channels, i.e. highly interacting species that derive } \\
\text { most of their energy or biomass from the same basal species } \\
\text { are common structures in food webs (Rooney and McCann } \\
\text { 2012) and the pattern of top predators coupling different } \\
\text { energy channels is an ecosystem scale structure that has } \\
\text { been found repeatedly (McCann and Rooney 2009; Rooney } \\
\text { et al. 2006). }\end{array}$ & $\begin{array}{l}\text { Certain motifs are likely to affect the stability of the system and } \\
\text { thus the robustness to invasion. }\end{array}$ \\
\hline Modularity & $\begin{array}{l}\text { Obese and inflammatory bowel disease microbiomes, viewed through } \\
\text { their metabolic networks, were found to have reduced modularity } \\
\text { (Greenblum et al. 2012) } \\
\text { Modularity of cecum (gut) from metagenomics and metaboliomics } \\
\text { (McHardy et al. 2013) }\end{array}$ & $\begin{array}{l}\text { Invasion experiments into simulated food webs found lower } \\
\text { modularity made the community more susceptible to } \\
\text { invasion (Lurgi et al. 2014) }\end{array}$ & $\begin{array}{l}\text { Reduced modularity in microbiome communities is posited to } \\
\text { be associated to lower variation in the environment } \\
\text { (Greenblum et al. 2012). These disturbed systems are also less } \\
\text { diverse and more susceptible to invasions. }\end{array}$ \\
\hline Node degree distribution & $\begin{array}{l}\text { are often scale-free (i.e. the node-degree distribution is described by } \\
\text { a power-law) but not always (Faust and Raes 2012) }\end{array}$ & are generally not scale-free (Dunne 2005; Zhou et al. 2010) & $\begin{array}{l}\text { Scale-free are generally less sensitive to random node removal } \\
\text { except when a hub node is removed, which implies that if an } \\
\text { invader affects a hub node then it is more likely to establish }\end{array}$ \\
\hline $\begin{array}{l}\text { Connectance } \\
\text { (connectedness) }\end{array}$ & $\begin{array}{l}\text { Host-associated microbiome networks (across several body sites and } \\
\text { constructed with positive and negative interactions) showed higher } \\
\text { connectedness (and network density) than soil networks (Faust et al. } \\
\text { 2015). }\end{array}$ & $\begin{array}{l}\text { Higher connectance has been found to decrease invasion } \\
\text { success (Dunne et al. 2002; Romanuk et al. 2009); however, } \\
\text { in some cases, larger food webs with low connectance (and } \\
\text { high modularity) can be robust to invasion (Lurgi et al. 2014). } \\
\text { Generally, though, it is thought that higher connectance is } \\
\text { more resistant to invasion (Smith-Ramesh et al. 2016). }\end{array}$ & Higher connectedness is believed to help prevent invasions \\
\hline $\begin{array}{l}\text { Average shortest path length } \\
\text { (AL) }\end{array}$ & Mostly have small AL (Faust and Raes 2012) & $\begin{array}{l}\text { Food webs have been found to have short path lengths } \\
\text { (Dunne 2006M; Montoya et al. 2002) }\end{array}$ & $\begin{array}{l}\text { Small AL increases the networks' responsiveness to } \\
\text { perturbations, i.e. faster return (Faust and Raes 2012; Zhou et } \\
\text { al. 2010) }\end{array}$ \\
\hline $\begin{array}{l}\text { Hub nodes } \\
\text { (core taxa, keystone and } \\
\text { foundational species) }\end{array}$ & $\begin{array}{l}\text { Identification of keystone and foundational species using network } \\
\text { inference and time series metagenomics data (Fisher and Mehta } \\
\text { 2014) and (Trosvik and de Muinck 2015) } \\
\text { Experimental example: } \\
\text { A low-abundance biofilm species orchestrates periodontal disease }\end{array}$ & $\begin{array}{l}\text { Removal of keystone species and fragmentation of food } \\
\text { webs can destabilise food webs (Solé and Montoya 2001; } \\
\text { Proulx et al. 2005) }\end{array}$ & $\begin{array}{l}\text { If an invader removes a keystone species or a foundational } \\
\text { species this can have strong effects on the community } \\
\text { Note: the trophic position into which the invader enters } \\
\text { matters to the initial phases of the introduction (Romanuk et al. } \\
\text { 2009) }\end{array}$ \\
\hline
\end{tabular}




\begin{tabular}{|c|c|c|c|}
\hline & (Hajishengallis et al. 2011) & & \\
\hline $\begin{array}{l}\text { Weak vs. strong interactions } \\
\text { (links) }\end{array}$ & $\begin{array}{l}\text { We did not find any direct investigations of this however it is } \\
\text { mentioned in (Foster et al. 2008) }\end{array}$ & $\begin{array}{l}\text { Weak interactions are generally stabilising while strong } \\
\text { interactions are generally destabilising (Gellner and Mccann } \\
\text { 2016). Trade-offs (e.g., competition versus edibility) can } \\
\text { readily produce these strong-weak associations. High } \\
\text { frequency of weak interactions is stabilising (Jacquet et al. } \\
\text { 2016) }\end{array}$ & $\begin{array}{l}\text { The new weak interactions (links) created by an invader can } \\
\text { affect stability of the resident community }\end{array}$ \\
\hline $\begin{array}{l}\text { Distribution } \\
\text { of positive and competitive } \\
\text { interactions }\end{array}$ & $\begin{array}{l}\text { Negative interaction networks have been found to be less connected } \\
\text { than the positive interactions of the same networks. Also, host- } \\
\text { associated networks contained more positive edges than soil } \\
\text { microbial communities (Faust et al. 2015; Friedman and Alm 2012) }\end{array}$ & $\begin{array}{l}\text { Invasion resistance arises in strongly interacting species-rich } \\
\text { model competition communities (Case 1990) } \\
\text { Transitive (hierarchical) competitive interactions are less } \\
\text { stable than intransitive (cyclical) networks of competitive } \\
\text { interactions (Petraitis 1979). } \\
\text { Invading species that feed on resident competitors are more } \\
\text { effective at entering a new system than species that only } \\
\text { compete with local species (Hall 2011) }\end{array}$ & $\begin{array}{l}\text { Invaders that compete with resident species can create } \\
\text { different structures and whether they create transitive or } \\
\text { intransitive structures could affect their invasion success. } \\
\text { Predatory or parasitic strategies are likely to lead to more } \\
\text { successful invasions than competitive strategies. }\end{array}$ \\
\hline
\end{tabular}

Table 1 


\section{References}

van den Abbeele, P. et al., 2013. Prebiotics, faecal transplants and microbial network units to stimulate biodiversity of the human gut microbiome. Microbial Biotechnology, 6(4), pp.335-340.

Abedon, S.T., 2014. Phage Therapy: Eco-Physiological Pharmacology. Scientifica, pp.1-30.

Abrams, P.A., 2000. The Evolution of Predator-Prey Interactions: Theory and Evidence. Annual Review of Ecology, Evolution, and Systematics, 31, pp.79-105.

Acheson, D.W.K. et al., 1998. In vivo transduction with shiga toxin 1-encoding phage. Infection and Immunity, 66(9), pp.4496-4498.

Aebersold, R. and Mann, M., 2016. Mass-spectrometric exploration of proteome structure and function. Nature, 537(7620), pp.347-355.

Aguirre, M. et al., 2015. Diet drives quick changes in the metabolic activity and composition of human gut microbiota in a validated in vitro gut model. Research in Microbiology, 167(October), pp.1-12.

Aguirre, M. et al., 2014. In vitro characterization of the impact of different substrates on metabolite production, energy extraction and composition of gut microbiota from lean and obese subjects. PLOS ONE, 9(11), pp.1-23.

Alizon, S., 2013. Parasite co-transmission and the evolutionary epidemiology of virulence. Evolution, 67(4), pp.921-933.

Alizon, S. and van Baalen, M., 2008. Acute or chronic? Within-host models with immune dynamics, infection outcome, and parasite evolution. The American Naturalist, 172(6), pp.E244-56.

Alizon, S. and Michalakis, Y., 2015. Adaptive virulence evolution: The good old fitness-based approach. Trends in Ecology and Evolution, 30(5), pp.248-254.

Alizon, S., de Roode, J.C. and Michalakis, Y., 2013. Multiple infections and the evolution of virulence. Ecology Letters, 16, pp.556-567.

Allen, E.K. et al., 2014. Characterization of the nasopharyngeal microbiota in health and during rhinovirus challenge. Microbiome, pp.1-11.

Allen-Vercoe, E. et al., 2012. A Canadian Working Group report on fecal microbial therapy: Microbial ecosystems therapeutics. Canadian Jouranl of Gastroenterology, 26(7), pp.457-462.

Allen-Vercoe, E., 2013. Bringing the gut microbiota into focus through microbial culture: recent progress and future perspective. Current opinion in microbiology, 16(5), pp.625-9.

Allen-Vercoe, E. and Petrof, E.O., 2013. Artificial stool transplantation: progress towards a safer, more effective and acceptable alternative. Expert Rev Gastroenterol Hepatol, 7(4), pp.291-293.

Allesina, S. and Levine, J.M., 2011. A competitive network theory of species diversity. Proceedings of the National Academy of Sciences of the United States of America, 108(14), pp.5638-5642.

Allison, S.D. and Martiny, J.B.H., 2008. Resistance, resilience, and redundancy in microbial communities. Proceedings of the National Academy of Sciences, 105 Suppl(Supplement 1), pp.11512-9.

Amsellem, L. Brouat C., Duron O., Porter S.S., Vilcinskas A. \& Facon B. (in press). Importance of microorganisms to macroorganisms invasions - Is the essential invisible to the eye? Adv. Ecol. Res., 56.

André, J.-B. and Day, T., 2005. The effect of disease life history on the evolutionary emergence of novel pathogens. Proceedings of the Royal Society B: Biological Sciences, 272(1575), pp.1949-56.

Antonopoulos, D. a. et al., 2009. Reproducible community dynamics of the gastrointestinal microbiota following antibiotic perturbation. Infection and Immunity, 77(6), pp.2367-2375.

Antonovics, J., 1968. Evolution in closely adjacent plant populations. Heredity, 23(4), pp.507-524.

Antonovics, J., 1976. The Nature of Limits to Natural Selection. Annals of the Missouri Botanical Garden, 63(2), pp.224-247.

Arumugam, M. et al., 2011. Enterotypes of the human gut microbiome. Nature, 473(7346), pp.174-80.

van Baalen, M. and Jansen, V.A.A., 2001. Dangerous liaisons: the ecology of private interest and common good. Oikos, 95(2), pp.211-224.

Bäckhed, F. et al., 2012. Defining a Healthy Human Gut Microbiome: Current Concepts, Future Directions, and Clinical Applications. Cell Host and Microbe, 12(5), pp.611-622.

Baines, S.D. et al., 2013. Mixed infection by Clostridium difficile in an in vitro model of the human gut. Journal of Antimicrobial Chemotherapy, 68(5), pp.1139-1143.

Ball, G.H., 1943. Parasitism and Evolution. The American naturalist, 77(771), pp.345-364.

Balzan, S. et al., 2007. Bacterial translocation: overview of mechanisms and clinical impact. Journal of 
gastroenterology and hepatology, 22(4), pp.464-471.

Begley, M., Hill, C. and Gahan, C.G.M., 2006. Bile Salt Hydrolase Activity in Probiotics Bile Salt Hydrolase Activity in Probiotics. Applied and Environmental Microbiology., 72(3), pp.1729-1738.

Berlow, E.L. et al., 2004. Interaction strengths in food webs: issues and opportunities. Journal of Animal Ecology, 73(3), pp.585-598.

Berry, D. and Widder, S., 2014. Deciphering microbial interactions and detecting keystone species with co-occurrence networks. Frontiers in Microbiology, 5(219), pp.1-14.

Best, K. et al., 2015. Computational analysis of stochastic heterogeneity in PCR amplification efficiency revealed by single molecule barcoding. Scientific Reports, 5, p.14629.

Bevan, M.J. and Fink, P.J., 2001. The CD8 response on autopilot. Nature immunology, 2(5), pp.381-2.

Bhattarai, N. and Stapleton, J.T., 2012. GB virus C: The good boy virus? Trends in Microbiology, 20(3), pp.124-130.

Biggs, H.M. et al., 2014. Invasive salmonella infections in areas of high and low malaria transmission intensity in Tanzania. Clinical Infectious Diseases, 58(5), pp.638-647.

Bolnick, D.I. et al., 2014. Major Histocompatibility Complex class Ilb polymorphism influences gut microbiota composition and diversity. Molecular Ecology, 23(19), pp.4831-4845.

Boon, E. et al., 2014. Interactions in the microbiome: Communities of organisms and communities of genes. FEMS Microbiology Reviews, 38(1), pp.90-118.

Boskey, E.R. et al., 2001. Origins of vaginal acidity: high D/L lactate ratio is consistent with bacteria being the primary source. Human Reproduction, 16(9), pp.1809-1813.

Boyd, E.F., 2012. Bacteriophage-Encoded Bacterial Virulence Factors and Phage-Pathogenicity Island Interactions. Advances in Virus Research,1st ed. 82: 91-118, Elsevier.

Broderick, N.A., 2015. A common origin for immunity and digestion. Frontiers in Immunology, 6, pp.1-3.

Brooks, J.P. et al., 2015. The truth about metagenomics: quantifying and counteracting bias in 16S rRNA studies. BMC Microbiology, 15(1), p.66.

Brown, S.P. et al., 2006. Ecology of Microbial Invasions: Amplification Allows Virus Carriers to Invade More Rapidly When Rare. Current Biology, 16(20), pp.2048-2052.

Brown, S.P., Cornforth, D.M. and Mideo, N., 2012. Evolution of virulence in opportunistic pathogens: generalism, plasticity, and control. Trends in microbiology, 20(7), pp.336-42.

Brown, S.P. and Grenfell, B.T., 2001. An unlikely partnership: parasites, concomitant immunity and host defence. Proceedings of the Royal Society B: Biological Sciences, 268, pp.2543-2549.

Brüssow, H. et al., 2004. Phages and the Evolution of Bacterial Pathogens: from Genomic Rearrangements to Lysogenic Conversion. Microbiology and molecular biology reviews, 68(3), pp.560-602.

Bucci, V. et al., 2016. MDSINE: Microbial Dynamical Systems INference Engine for microbiome timeseries analyses. Genome Biology, 17(1), p.121.

Bucci, V. and Xavier, J.B., 2014. Towards predictive models of the human gut microbiome. Journal of Molecular Biology, 426(23), pp.3907-3916.

Buffie, C.G. et al., 2015. Precision microbiome reconstitution restores bile acid mediated resistance to Clostridium difficile. Nature, 517(7533), pp.205-8.

Buffie, C.G. and Pamer, E.G., 2013. Microbiota-mediated colonization resistance against intestinal pathogens. Nat Rev Immunol, 13(11), pp.790-801.

Burns, J.H. et al., 2013. Greater sexual reproduction contributes to differences in demography of invasive plants and their noninvasive relatives. Ecology, 94(5), pp.995-1004.

Burns, J.H., 2006. Relatedness and environment affect traits associated with invasive and noninvasive. Ecological Applications, 16(4), pp.1367-1376.

Cabreiro, F., 2016. Metformin joins forces with microbes. Cell Host and Microbe, 19(1), p.1.

Callaway, R.M. and Ridenour, W.M., 2004. Novel weapons: Invasive success and the evolution of increased competitive ability. Frontiers in Ecology and the Environment, 2(8), pp.436-443.

Caporaso, J.G. et al., 2011. Moving pictures of the human microbiome. Genome Biology, 12(5), p.R50.

Carboni, M. et al., 2016. What it takes to invade grassland ecosystems: Traits, introduction history and filtering processes. Ecology Letters, 19(3), pp.219-229.

Carrington, M. et al., 1999. Genetics of HIV-1 infection: Chemokine receptor CCR5 polymorphism and its consequences. Human Molecular Genetics, 8(10), pp.1939-1945.

Carrolo, M. et al., 2010. Prophage spontaneous activation promotes DNA release enhancing biofilm formation in Streptococcus pneumoniae. PLOS ONE, 5(12). 
Case, T.J., 1990. Invasion resistance arises in strongly interacting species-rich model competition communities. Proceedings of the National Academy of Sciences of the United States of America, 87(24), pp.9610-4.

Case, T.J. and Bolger, D.T., 1991. The role of introduced species in shaping the abundance and distribution of island reptiles. Evolutionary Ecology, 5, pp.272-290.

Chassaing, B. et al., 2015. Dietary emulsifiers impact the mouse gut microbiota promoting colitis and metabolic syndrome. Nature, 519(7541), pp.92-96.

Chaturongakul, S. and Ounjai, P., 2014. Phage-host interplay: examples from tailed phages and Gramnegative bacterial pathogens. Frontiers in microbiology, 5(August), p.442.

Chibani-chennou, S. et al., 2004. In Vitro and In Vivo Bacteriolytic Activities of. Society, 48(7), pp.25582569.

Cho, I. and Blaser, M.J., 2012. The human microbiome: at the interface of health and disease. Nature Reviews Genetics, 13(4), pp.260-270.

Chukkapalli, S.S. et al., 2015. Polymicrobial Oral Infection with Four Periodontal Bacteria Orchestrates a Distinct Inflammatory Response and Atherosclerosis in ApoE null Mice. PLoS ONE, pp.1-17.

Clemente, J.C. et al., 2012. The impact of the gut microbiota on human health: An integrative view. Cell, 148(6), pp.1258-1270.

Costello, E.K. et al., 2012. The Application of Ecological Theory Toward an Understanding of the Human Microbiome. Science, 336(6086), pp.1255-1262.

Coyte, K.Z., Schluter, J. and Foster, K.R., 2015. The ecology of the microbiome: Networks, competition, and stability. Science, 350(6261), pp.663-666.

Criswell, B.S. et al., 1969. Haemophilus vaginalis: vaginitis by inoculation from culture. Obstetrics and Gynecology, 33(2), pp.195-199.

Crowther, G.S. et al., 2016. Efficacy of vancomycin extended-dosing regimens for treatment of simulated Clostridium difficile infection within an in vitro human gut model. The Journal of antimicrobial chemotherapy, (January), pp.986-991.

D’Acunto, B. et al., 2015. Modeling multispecies biofilms including new bacterial species invasion. Mathematical Biosciences, 259, pp.20-26.

Dalton, T. et al., 2011. An in vivo polymicrobial biofilm wound infection model to study interspecies interactions. PLOS ONE, 6(11).

Danielsson, D., Teigen, P.K. and Moi, H., 2011. The genital econiche: focus on microbiota and bacterial vaginosis. Annals of the New York Academy of Sciences, 1230, pp.48-58.

Datcu, R. et al., 2013. Vaginal microbiome in women from Greenland assessed by microscopy and quantitative PCR. BMC infectious diseases, 13, p.480.

David P., Thébault E., Anneville O., Duyck P.-F., Chapuis E. \& Loeuille N. (in press). Impacts of invasive species on food webs: a review of empirical data. Adv. Ecol. Res., 56.

Delong, E.F., 2014. Alien invasions and gut island biogeography. Cell, 159(2), pp.233-235.

Delwart, E., 2013. A roadmap to the human virome. PLoS pathogens, 9(2), p.e1003146.

Derrien, M. and van Hylckama Vlieg, J.E.T., 2015. Fate, activity, and impact of ingested bacteria within the human gut microbiota. Trends in Microbiology, 23(6), pp.354-366.

Dethlefsen, L., McFall-Ngai, M. and Relman, D.A., 2007. An ecological and evolutionary perspective on human-microbe mutualism and disease. Nature, 449(7164), pp.811-8.

Detmer, A. and Glenting, J., 2006. Live bacterial vaccines--a review and identification of potential hazards. Microbial cell factories, 5(iii), p.23.

Donohue, I. et al., 2016. Navigating the complexity of ecological stability. Ecology Letters, pp.1172-1185.

Donohue, I. et al., 2013. On the dimensionality of ecological stability. Ecology letters, 16(4), pp.421-9.

Drake, J.A., 1990. Communities as assembled structures: Do rules govern pattern? Trends in Ecology and Evolution, 5(5), pp.159-164.

Duan, K. et al., 2003. Modulation of Pseudomonas aeruginosa gene expression by host microflora through interspecies communication. Molecular Microbiology, 50(5), pp.1477-1491.

Duerkop, B. A. et al., 2012. A composite bacteriophage alters colonization by an intestinal commensal bacterium. Proceedings of the National Academy of Sciences of the United States of America, 109(43), pp.17621-6.

Dunne, J., 2006. The Network Structure of Food Webs. In Ecological Networks. Linking structure to dynamics in food webs. p. 386.

Dunne, J.A. et al., 2002. Food-web structure and netv cork theory: The role of connectance and size. 
Proceedings of the National Academy of Sciences, 99(20), pp.12917-12922.

Dunne, J.A., 2005. The Network Structure of Food Webs. Ecological Networks: Linking Structure to Dynamics in Food Webs, 23(March), pp.27-86.

Duyck, P.F. et al., 2006. Importance of competition mechanisms in successive invasions by polyphagous Tephritids in la reunion. Ecology, 87(7), pp.1770-1780.

Duyck, P.F., David, P. and Quilici, S., 2004. A review of relationships between interspecific competition and invasions in fruit flies (Diptera: Tephritidae). Ecological Entomology, 29(5), pp.511-520.

Eaton, M.J. et al., 2014. Testing metapopulation concepts: Effects of patch characteristics and neighborhood occupancy on the dynamics of an endangered lagomorph. Oikos, 123(6), pp.662676.

Edlin, G. and Bitner, R., 1977. Reproductive fitness of P1, P2 and Mu lysogens of Escherichia coli. Journal of Virology, 21(2), pp.560-564.

Edlin, G., Lin, L. and Kudrna, R., 1975. Gamma lysogens of E. coli reproduce more rapidly than nonlysogens. Nature, 255, pp.735-737.

Edlund, A. et al., 2013. An in vitro biofilm model system maintaining a highly reproducible species and metabolic diversity approaching that of the human oral microbiome. Microbiome, 1(1), p.25.

Eichmann, K., 2008. The Network Collective: Rise and Fall of a Scientific Paradigm, Birkhäuser Basel.

Elton, C.S., 1958. The ecology of invasions by animals and plants, The University of Chicago Press.

Enault, F. et al., 2016. Phages rarely encode antibiotic resistance genes: a cautionary tale for virome analyses. The ISME journal, June 21.

Ezenwa, V.O. and Jolles, A.E., 2015. Opposite effects of anthelmintic treatment on microbial infection at individual versus population scales. Science, 347(6218), pp.175-177.

Facon, B. et al., 2008. High Genetic Variance in Life-History Strategies within Invasive Populations by Way of Multiple Introductions. Current Biology, 18(5), pp.363-367.

Facon, B. et al., 2005. Hybridization and invasiveness in the freshwater snail Melanoides tuberculata: Hybrid vigour is more important than increase in genetic variance. Journal of Evolutionary Biology, 18(3), pp.524-535.

Faith, J.J. et al., 2013. The long-term stability of the human gut microbiota. Science, 341(6141), p.1237439.

Faust, K. et al., 2015. Cross-biome comparison of microbial association networks. Frontiers in Microbiology, 6, p.1200.

Faust, K. and Raes, J., 2012. Microbial interactions: from networks to models. Nature reviews. Microbiology, 10(8), pp.538-50.

Fayol-Messaoudi, D. et al., 2005. pH- , Lactic Acid- , and Non-Lactic Acid-Dependent Activities of Probiotic Lactobacilli against Salmonella enterica Serovar Typhimurium. Applied and Environmental Microbiology, 71(10), pp.6008-6013.

Fenton, A. and Perkins, S.E., 2010. Applying predator-prey theory to modelling immune-mediated, withinhost interspecific parasite interactions. Parasitology, 137(6), pp.1027-38.

Filippov, A.A. et al., 2011. Bacteriophage-resistant mutants in yersinia pestis: Identification of phage receptors and attenuation for mice. PLOS ONE, 6(9).

Fisher, C.K. and Mehta, P., 2014a. Identifying Keystone Species in the Human Gut Microbiome from Metagenomic Timeseries using Sparse Linear Regression. PLoS ONE, 9(7), pp.1-17.

Fisher, C.K. and Mehta, P., 2014b. The transition between the niche and neutral regimes in ecology. Proceedings of the National Academy of Sciences, 111(36), pp.13111-13116. Available at:

Flipse, J., Wilschut, J. and Smit, J.M., 2013. Molecular mechanisms involved in antibody-dependent enhancement of dengue virus infection in humans. Traffic, 14(1), pp.25-35.

Ford, S.A. and King, K.C., 2016. Harnessing the Power of Defensive Microbes: Evolutionary Implications in Nature and Disease Control. PLoS Pathogens, 12(4), pp.1-12.

Foster, J.A., Krone, S.M. and Forney, L.J., 2008. Application of ecological network theory to the human microbiome. Interdisciplinary perspectives on infectious diseases, 2008, p.839501.

Foster, K.R. and Bell, T., 2012. Competition, not cooperation, dominates interactions among culturable microbial species. Current Biology, 22(19), pp.1845-1850.

Foster, K.R. and Wenseleers, T., 2006. A general model for the evolution of mutualisms. Journal of Evolutionary Biology, 19(4), pp.1283-1293.

Foxman, E.F. and Iwasaki, A., 2011. Genome-virome interactions: examining the role of common viral infections in complex disease. Nature reviews. Microbiology, 9(4), pp.254-64. 
Franzén, O. et al., 2015. Improved OTU-picking using long-read 16S rRNA gene amplicon sequencing and generic hierarchical clustering. Microbiome, 3, p.43.

Friedman, J. and Alm, E.J., 2012. Inferring Correlation Networks from Genomic Survey Data. PLoS computational biology, 8(9), pp.1-11.

Friman, V.P. et al., 2016. Pre-adapting parasitic phages to a pathogen leads to increased pathogen clearance and lowered resistance evolution with Pseudomonas aeruginosa cystic fibrosis bacterial isolates. Journal of Evolutionary Biology, 29(1), pp.188-198.

Furuse, K. et al., 1983. Distribution of RNA coliphages in Senegal, Ghana, and Madagascar. Microbiology and Immunology, 27(4), pp.347-358.

Gajer, P. et al., 2012. Temporal dynamics of the human vaginal microbiota. Science translational medicine, 4(132), p.132ra52.

Gallien, L. et al., 2014. Contrasting the effects of environment, dispersal and biotic interactions to explain the distribution of invasive plants in alpine communities. Biological Invasions, 17(5), pp.1407-1423.

Gama, J.A. et al., 2013. Temperate Bacterial Viruses as Double-Edged Swords in Bacterial Warfare. PLOS ONE, 8(3).

Gao, J., Barzel, B. and Barabási, A.-L., 2016. Universal resilience patterns in complex networks. Nature, 530(7590), pp.307-312.

Gardner, H.L. and Dukes, C.D., 1955. Haemophilus vaginalis vaginitis: a newly defined specific infection previously classified "nonspecific" vaginitis. American Journal of Obstetrics and Gynecology, 69(5), pp.962-976.

Gellner, G. and Mccann, K.S., 2016. The Consistent Role of Weak and Strong Interactions in High and Low-Diversity Food Webs. Nature Communications, 7(11180), pp.1-7. Available at:

Geva-Zatorsky, N. et al., 2015. In vivo imaging and tracking of host-microbiota interactions via metabolic labeling of gut anaerobic bacteria. Nature Medicine, 21(9), pp.1091-1100.

Gibson, T.E. et al., 2015. On the Origins and Control of Community Types in the Human Microbiome. arXiv, pp.1-84. Available at: http://arxiv.org/abs/1506.05171.

Gilligan, C.A. and Van Den Bosch, F., 2008. Epidemiological Models for Invasion and Persistence of Pathogens. Annu. Rev. Phytopathol, 46, pp.385-418.

Girard, M.P. et al., 2006. A review of vaccine research and development: Human enteric infections. Vaccine, 24(15), pp.2732-2750.

Goodman, A.L., 2014. In Vivo and animal models of the human gut microbiome. In J. R. Marchesi, ed. The Human Microbiota and Microbiome. CABI Publishing, p. 208.

Goodnight, K.F., 1992. The Effect of Stochastic Variation on Kin Selection in a Budding-Viscous Population. The American naturalist, 140(6), pp.1028-1040.

Graf, J., Kikuchi, Y. and Rio, R.V.M., 2006. Leeches and their microbiota: naturally simple symbiosis models. Trends in Microbiology, 14(8), pp.365-371.

Graham, A.L., 2008. Ecological rules governing helminth-microparasite coinfection. Proceedings of the National Academy of Sciences of the United States of America, 105(2), pp.566-70.

Greenblum, S., Turnbaugh, P.J. and Borenstein, E., 2012. Metagenomic systems biology of the human gut microbiome reveals topological shifts associated with obesity and inflammatory bowel disease. Proceedings of the National Academy of Sciences, 109(2), pp.594-599.

Grice, E. a and Segre, J. a, 2011. The skin microbiome. Nature reviews. Microbiology, 9(4), pp.244-253.

Griffiths, E.C. et al., 2014. Analysis of a summary network of co-infection in humans reveals that parasites interact most via shared resources. Proceedings of the Royal Society B: Biological Sciences, 281, p.20132286.

Hajishengallis, G. et al., 2011. Article Low-Abundance Biofilm Species Orchestrates Inflammatory Periodontal Disease through the Commensal Microbiota and Complement. Cell Host and Microbe, 10(5), pp.497-506.

Hajishengallis, G. and Lamont, R.J., 2012. Beyond the red complex and into more complexity: The polymicrobial synergy and dysbiosis (PSD) model of periodontal disease etiology. Molecular Oral Microbiology, 27(6), pp.409-419.

Hall, R.J., 2011. Eating the competition speeds up invasions. Biology Letters, 7(2), pp.307-311.

Hamilton, P.T. and Perlman, S.J., 2013. Host Defense via Symbiosis in Drosophila. PLoS Pathogens, 9(12), pp.1-4.

Hamilton, W., 1980. Sex Versus Non-Sex Versus ParasiteOikos. Oikos, 35(2), pp.282-290.

Hamilton, W.D., 1964. The genetical evolution of social behaviour. I. Journal of Theoretical Biology, 7(1), 
pp.1-16.

Hammer, B.K. and Bassler, B.L., 2003. Quorum sensing controls biofilm formation in Vibrio cholerae. Molecular Microbiology, 50(1), pp.101-114.

Harcombe, W.R. et al., 2016. Adding biotic complexity alters the metabolic benefits of mutualism. Evolution, 70(8), pp.1871-1881.

Harriott, M.M. and Noverr, M.C., 2011. Importance of Candida-bacterial polymicrobial biofilms in disease. Trends in Microbiology, 19(11), pp.557-563.

Hartfield, M. and Alizon, S., 2014. Epidemiological Feedbacks Affect Evolutionary Emergence of Pathogens. The American Naturalist, 183(4), pp.E105-17.

Hartman, A.L. et al., 2009. Human gut microbiome adopts an alternative state following small bowel transplantation. Proceedings of the National Academy of Sciences of the United States of America, 106(40), pp.17187-17192.

Hastings, A., 1978. Spatial heterogeneity and the stability of predator-prey systems: Predator-mediated coexistence. Theoretical Population Biology, 14(3), pp.380-395.

Heinsen, F.A. et al., 2015. Dynamic changes of the luminal and mucosaassociated gut microbiota during and after antibiotic therapy with paromomycin. Gut Microbes, 6(4), pp.243-254.

Hickey, R.J. et al., 2015. Vaginal microbiota of adolescent girls prior to the onset of menarche resemble those of reproductive-age women. $m B i o, 6(2)$, pp.1-14.

Hofbauer, J. and Schreiber, S.J., 2010. Robust permanence for interacting structured populations. Journal of Differential Equations, 248(8), pp.1955-1971. Available at:

Holling, C.S., 1959. Some characteristics of simple types of predation and parasitism. The Canadian Entomologist, $\mathrm{XCl}(7)$, p.385.

Holt, R.D., Grover, J., and Tilman, D. T., 1994. Simple rules for interspecific dominance in systems with exploitaitve and apparent competition, The American Naturalist, 144(5), pp.741-771.

Holt, R. D. and Hochberg, M., 2001. Indirect interactions, community modules and biological control: a theoretical perspective. In P. C. Q. E. Wajnberg, John K. Scott, ed. Evaluating indirect ecological effects of biological control. CABI Publishing, p. 261.

Holt, R.D. and Barfield, M., 2006. Within-host pathogen dynamics : Some ecological and evolutionary consequences of transients, dispersal mode, and within-host spatial heterogeneity. DIMACS Series in Discrete Mathematics and Theoretical Computer Science, 71, pp.45-66.

Holt, R.D. and Dobson, A.P., 2006. Extending the principles of community ecology to address the epidemiology of host-pathogen systems. In S. K. Collinge and C. Ray, eds. Disease Ecology: community structure and pathogen dynamics. Oxford: Oxford University Press, pp. 6-27.

Hooper, L. V et al., 2003. Angiogenins: a new class of microbicidal proteins involved in innate immunity. Nature immunology, 4(3), pp.269-273.

Horz, H.-P., 2015. Archaeal Lineages within the Human Microbiome: Absent, Rare or Elusive? Life, 5(2), pp.1333-1345.

Hufbauer, R.A. et al., 2012. Anthropogenically induced adaptation to invade (AIAI): Contemporary adaptation to human-altered habitats within the native range can promote invasions. Evolutionary Applications, 5(1), pp.89-101.

Hufbauer, R.A. et al., 2013. Role of propagule pressure in colonization success: Disentangling the relative importance of demographic, genetic and habitat effects. Journal of Evolutionary Biology, 26(8), pp.1691-1699.

Huffnagle, G.B. and Noverr, M.C., 2013. The emerging world of the fungal microbiome. Trends in Microbiology, 21(7), pp.334-341.

Hunt, K.M. et al., 2011. Characterization of the diversity and temporal stability of bacterial communities in human milk. PLoS ONE, 6(6), pp.1-8.

Inal, J.M., 2003. Phage therapy: a reappraisal of bacteriophages as antibiotics. Archivum immunologiae et therapiae experimentalis, 51, pp.237-244.

Inderjit, Cadotte, M.W. and Colautti, R.I., 2005. The ecology of biological invasions: past, present and future. In Birkhauser, ed. Invasive Plants: Ecological and Agricutural Aspects. Verlag, Switzerland, p. 19.

Ives, A.R. and Carpenter, S.R., 2007. Stability and diversity of ecosystems. Science, 317(5834), pp.5862.

Jackson M.C., Wasserman R.J., Grey J., Ricciardi A., Dick J.T.A. \& Alexander M.E. (in press). Novel and disrupted trophic links following invasion in freshwater ecosystems. Adv. Ecol. Res., 56. 
Jacquet, C. et al., 2016. The complexity-stability relationship in empirical ecosystems. Nature Communications, 7, p.12573.

Jaenlke, J., 1978. A hypothesis to account for the maintenance of sex within populations. Evolutionary Theory, 94, pp.191-194.

Jakobsson, H.E. et al., 2010. Short-term antibiotic treatment has differing long- term impacts on the human throat and gut microbiome. PLOS ONE, 5(3).

Jansen, V. a a and van Baalen, M., 2006. Altruism through beard chromodynamics. Nature, 440(7084), pp.663-666.

Jenkins, C. and Keller, S.R., 2011. A phylogenetic comparative study of preadaptation for invasiveness in the genus Silene (Caryophyllaceae). Biological Invasions, 13(6), pp.1471-1486.

Jeschke, J.M., 2014. General hypotheses in invasion ecology. Diversity and Distributions, 20(11), pp.1229-1234.

Johnson, S. et al., 2014. Trophic coherence determines food-web stability. Proceedings of the National Academy of Sciences, 1(50), pp.1-6.

Jordán, F. et al., 2015. Diversity of key players in the microbial ecosystems of the human body. Scientific Reports, 5, p.15920.

Jover, L.F., Cortez, M.H. and Weitz, J.S., 2013. Mechanisms of multi-strain coexistence in host-phage systems with nested infection networks. Journal of Theoretical Biology, 332, pp.65-77. Available at:

Jung, J.Y. et al., 2011. Metagenomic analysis of kimchi, a Traditional Korean fermented food. Applied and Environmental Microbiology, 77(7), pp.2264-2274.

Kaewsrichan, J., Peeyananjarassri, K. and Kongprasertkit, J., 2006. Selection and identification of anaerobic lactobacilli producing inhibitory compounds against vaginal pathogens. FEMS Immunology and Medical Microbiology, 48(1), pp.75-83.

Kaur, T. et al., 2012. Immunocompatibility of bacteriophages as nanomedicines. Journal of Nanotechnology, 2012(i).

Keen, E.C., 2012. Phage therapy: Concept to cure. Frontiers in Microbiology, 3, pp.1-3.

Kéfi, S. et al., 2012. More than a meal... integrating non-feeding interactions into food webs. Ecology Letters, 15(4), pp.291-300.

Keller, S.R. and Taylor, D.R., 2008. History, chance and adaptation during biological invasion: Separating stochastic phenotypic evolution from response to selection. Ecology Letters, 11(8), pp.852-866.

Kennedy, K. et al., 2014. Evaluating bias of Illumina-based bacterial 16S rRNA gene profiles. Applied and Environmental Microbiology, 80(18), pp.5717-5722.

Kennedy, T. a et al., 2002. Biodiversity as a barrier to ecological invasion. Nature, 417(6889), pp.636638.

Kerr, B. et al., 2002. Local dispersal promotes biodiversity in a real-life game of rock-paper-scissors. Nature, 418(6894), pp.171-174.

Kew, O.M. et al., 2005. Vaccine-derived polioviruses and the endgame strategy for global polio eradication. Annual review of microbiology, 59, pp.587-635.

Khanna, S. et al., 2016. A novel microbiome therapeutic increases gut microbial diversity and prevents recurrent Clostridium difficile infection. Journal of Infectious Diseases, 214(2), p.173.

Kiliç, A. O. et al., 2001. Comparative study of vaginal Lactobacillus phages isolated from women in the United States and Turkey: prevalence, morphology, host range, and DNA homology. Clin diagn lab immunol, 8(1), pp.31-9.

King, K.C. et al., 2016. Rapid evolution of microbe-mediated protection against pathogens in a worm host. The ISME Journal, 10(8), pp.1915-1924.

Koenig, J.E. et al., 2011. Succession of microbial consortia in the developing infant gut microbiome. Proceedings of the National Academy of Sciences of the United States of America, 108 Suppl, pp.4578-4585.

Koizumi, Y. and Iwami, S., 2014. Mathematical modeling of multi-drugs therapy: a challenge for determining the optimal combinations of antiviral drugs. Theoretical biology and medical modelling, $11(1)$, p.41.

Kubinak, J.L. et al., 2015. MHC variation sculpts individualized microbial communities that control susceptibility to enteric infection. Nature communications, 6, p.8642.

Kümmerli, R. et al., 2009. Limited dispersal, budding dispersal, and cooperation: An experimental study. Evolution, 63(4), pp.939-949.

Kurtz, Z.D. et al., 2015. Sparse and Compositionally Robust Inference of Microbial Ecological Networks. 
PLoS Computational Biology, 11(5), pp.1-25.

Kyriazakis, I., Tolkamp, B. and Hutchings, M., 1998. Towards a functional explanation for the occurrence of anorexia during parasitic infections. Animal behaviour, 56(2), pp.265-274.

Lang, J.M., Eisen, J.A. and Zivkovic, A.M., 2014. The microbes we eat: abundance and taxonomy of microbes consumed in a day's worth of meals for three diet types. Peer, 2, p.e659.

Law, R. and Morton, R.D., 1996. Permanence and the Assembly of Ecological Communities. Ecology, 77(3), pp.762-775.

Lawlor, L.R., 1979. Direct and indirect effects of n-species competition. Oecologia, 43(3), pp.355-364.

Lecuit, M. and Eloit, M., 2013. The human virome: new tools and concepts. Trends in microbiology, 21(10), pp.510-5.

Lee, W., van Baalen, M. and Jansen, V.A.A., 2012. An evolutionary mechanism for diversity in siderophore-producing bacteria. Ecology Letters, 15(2), pp.119-125.

Lee, W.-J. and Brey, P.T., 2013. How Microbiomes Influence Metazoan Development:Insights from History and Drosophila Modeling of Gut-Microbe Interactions. Annual Review of Cell and Developmental Biology, 29(1), pp.571-592.

Lemon, K.P. et al., 2012. Microbiota-Targeted Therapies : An Ecological Perspective. Science translational medicine, 4(137), p.RV5.

Van Lenteren, J.C. et al., 2003. Environmental risk assessment of exotic natural enemies used in inundative biological control. BioControl, 48, pp.3-38.

Leone, V. et al., 2015. Effects of diurnal variation of gut microbes and high-fat feeding on host circadian clock function and metabolism. Cell Host and Microbe, 17(5), pp.681-689.

Levin, B.R. and Bull, J.J., 2004. Population and evolutionary dynamics of phage therapy. Nature reviews. Microbiology, 2(2), pp.166-173.

Levin, B.R. and Bull, J.J., 1994. Short-sighted evolution and the virulence of pathogenic microorganisms. Trends in microbiology, 2(3), pp.76-81.

Levine, J.M. and Antonio, C.M.D., 1999. Elton revisited: a review of evidence linking diversity and invasibility. Oikos, 87(1), pp.15-26.

Ley, R.E., Peterson, D.A. and Gordon, J.I., 2006. Ecological and evolutionary forces shaping microbial diversity in the human intestine. Cell, 124(4), pp.837-848.

$\mathrm{Li}$, L. et al., 1998. Effect of feeding of a cholesterol-reducing bacterium, Eubacterium coprostanoligenes, to germ-free mice. Laboratory animal science, 48(3), pp.253-5.

$\mathrm{Li}, \mathrm{S}$. peng et al., 2015. The effects of phylogenetic relatedness on invasion success and impact: Deconstructing Darwin's naturalisation conundrum. Ecology Letters, 18(12), pp.1285-1292.

$\mathrm{Li}, \mathrm{S} . \mathrm{S}$. et al., 2016. Durable coexistence of donor and recipient strains after fecal microbiota transplantation. Science, 352(6285), pp.586-9.

Ling, Z. et al., 2013. The Restoration of the Vaginal Microbiota After Treatment for Bacterial Vaginosis with Metronidazole or Probiotics. Microbial Ecology, 65(3), pp.773-780.

Lively, C.M., 2010. A review of red queen models for the persistence of obligate sexual reproduction. Journal of Heredity, 101(SUPPL. 1), pp.13-20.

Lloyd-Smith, J.O. et al., 2009. Epidemic dynamics at the human-animal interface. Science, 326, pp.13627.

Loc-Carrillo, C. and Abedon, S.T., 2011. Pros and cons of phage therapy. Bacteriophage, 1(2), pp.111114.

Lockwood, J.L., Cassey, P. and Blackburn, T., 2005. The role of propagule pressure in explaining species invasions. Trends in Ecology and Evolution, 20(5), pp.223-228.

Loeuille, N., 2010. Influence of evolution on the stability of ecological communities. Ecology letters, 13(12), pp.1536-45.

Looft, T. and Allen, H.K., 2012. Collateral effects of antibiotics on mammalian gut microbiomes. Gut microbes, 3(5), pp.463-467.

Louda, S.M. et al., 2003. NonTarget Effects - The Achilles' Heel of Biological Control? Retrospective analysis to reduce risk associated with biocontrol introductions. Annual Review of Entomology, 48(1), pp.365-396.

Lozupone, C.A. et al., 2012. Diversity, stability and resilience of the human gut microbiota. Nature, 489, p.220.

Luciani, F. and Alizon, S., 2009. The evolutionary dynamics of a rapidly mutating virus within and between hosts: the case of hepatitis C virus. PLoS computational biology, 5(11), p.e1000565. 
Lurgi, M. et al., 2014. Network complexity and species traits mediate the effects of biological invasions on dynamic food webs. Frontiers in Ecology and Evolution, 2(July), pp.1-11.

Lurie-Weinberger, M.N. and Gophna, U., 2015. Archaea in and on the Human Body: Health Implications and Future Directions. PLoS Pathogens, 11(6), pp.1-8.

Lysenko, E.S. et al., 2010. Within-host competition drives selection for the capsule virulence determinant of streptococcus pneumoniae. Current Biology, 20(13), pp.1222-1226.

$\mathrm{Ma}, \mathrm{C}$. et al., 2016. Different effects of invader-native phylogenetic relatedness on invasion success and impact: a meta-analysis of Darwin's naturalization hypothesis. Proceedings of the Royal Society B: Biological Sciences, 283(1838), p.20160663.

Macfarlane, G.T. and Macfarlane, S., 2013. Manipulating the indigenous microbiota in humans: prebiotics, probiotics and synbiotics. In D. N. Fredricks, ed. The Human Microbiota: how microbial communities affect health and disease. Wiley-Blackwell, p. 389.

Machado, A. and Cerca, N., 2015. The influence of biofilm formation by Gardnerella vaginalis and other anaerobes on bacterial vaginosis. $J$ Infect Dis, 212, pp.1-17.

Machado, A., Jefferson, K.K. ay and Cerca, N., 2013. Interactions between Lactobacillus crispatus and bacterial vaginosis (BV)-associated bacterial species in initial attachment and biofilm formation. International journal of molecular sciences, 14(6), pp.12004-12012.

Maizels, R.M. and Yazdanbakhsh, M., 2003. Immune regulation by helminth parasites: cellular and molecular mechanisms. Nat Rev Immunol, 3(9), pp.733-744.

Marder, E. and Taylor, A.L., 2011. Multiple models to capture the variability in biological neurons and networks. Nature Neuroscience, 14(2), pp.133-138.

Margolis, E. and Levin, B.R., 2007. Within-host evolution for the invasiveness of commensal bacteria: an experimental study of bacteremias resulting from Haemophilus influenzae nasal carriage. The Journal of infectious diseases, 196(7), pp.1068-75.

Marino, S. et al., 2014. Mathematical modeling of primary succession of murine intestinal microbiota. Proceedings of the National Academy of Sciences, 111(1), pp.439-444.

Martiny, J.B.H. et al., 2015. Microbiomes in light of traits: A phylogenetic perspective. Science, 350(6261), p.aac9323.

Martz, S.-L.E. et al., 2015. Administration of defined microbiota is protective in a murine Salmonella infection model. Scientific Reports, 5, p.16094.

Massol F., Dubart M., Calcagno V., Cazelles K., Jacquet C., Kéfi S. \& Gravel D. (in press). Island biogeography of food webs. Adv. Ecol. Res., 56.

Mastropaolo, M.D. et al., 2005. Synergy in Polymicrobial Infections in a Mouse Model of Type 2 Diabetes †. Infection and Immunity, 73(9), pp.6055-6063.

Matsuoka, K. and Kanai, T., 2015. The gut microbiota and inflammatory bowel disease. Seminars in Immunopathology, 37(1), pp.47-55.

May, R.M., 1973. Stability and complexity in model ecosystems, Princeton Univ. Press.

May, R.M., 1972. Will a large complex system be stable? Nature, 238(5364), pp.413-414.

Mayer, A. et al., 2016. Diversity of immune strategies explained by adaptation to pathogen statistics. Proceedings of the National Academy of Sciences, 113(31), pp.8630-5.

McCann, K.S., 2012. Food Webs Monographs. S. A. Levin, ed., NJ: Princeton University Press.

McCann, K.S., 2000. The diversity-stability debate. Nature, 405(6783), pp.228-33.

McCann, K.S. and Gellner, G., 2012. Food chains and food web modules. In A. Hastings and L. Gross, eds. Encyclopedia of Theoretical Ecology. University of California Press, pp. 288-294.

McCann, K.S. and Rooney, N., 2009. The more food webs change, the more they stay the same. Philosophical transactions of the Royal Society of London. Series B, Biological sciences, 364(1524), pp.1789-801.

McDonald, J.A.K. et al., 2013. Evaluation of microbial community reproducibility, stability and composition in a human distal gut chemostat model. Journal of microbiological methods, 95(2), pp.167-74.

McDonald, J.A.K. et al., 2015. Simulating distal gut mucosal and luminal communities using packedcolumn biofilm reactors and an in vitro chemostat model. Journal of Microbiological Methods, 108, pp.36-44.

McHardy, I.H. et al., 2013. Integrative analysis of the microbiome and metabolome of the human intestinal mucosal surface reveals exquisite inter-relationships. Microbiome, 1(1), p.17.

McNally, L., Vale, P.F. and Brown, S.P., 2015. Microbiome engineering could select for more virulent pathogens. bioRxiv, Sep. 30. 
McSorley, H.J. and Maizels, R.M., 2012. Helminth infections and host immune regulation. Clinical Microbiology Reviews, 25(4), pp.585-608.

Meaden, S. and Koskella, B., 2013. Exploring the risks of phage application in the environment. Frontiers in microbiology, 4(November), p.358. Available at:

Médoc V., Firmat C., Sheath D.J., Pegg J., Andreou D. \& Britton J.R. (in press). Parasites and Biological Invasions: Predicting ecological alterations at levels from individual hosts to whole networks. Adv. Ecol. Res., 56.

Méthot, P.-O., 2012. Why do parasites harm their host? On the origin and legacy of Theobald Smith's "law of declining virulence"--1900-1980. History and philosophy of the life sciences, 34(4), pp.561601.

Michalakis, Y. et al., 1992. Pleiotropic Action of Parasites: How to be Good for the Host. Trends in Ecology and Evolution, 7(2), pp.59-62.

Mideo, N., 2009. Parasite adaptations to within-host competition. Trends in Parasitology, 25(6), pp.261-8.

Milinski, M. 1, 2006. The Major Histocompatibility Complex, Sexual Selection, and Mate Choice. Annual Review of Ecology, Evolution, and Systematics, 37, pp.159-186.

Milo, R. et al., 2002. Network motifs: simple building blocks of complex networks. Science, 298(5594), pp.824-7.

Minot, S. et al., 2013. Rapid evolution of the human gut virome. Proceedings of the National Academy of Sciences, 110(30), pp.12450-12455.

Minot, S. et al., 2011. The human gut virome: inter-individual variation and dynamic response to diet. Genome research, 21(10), pp.1616-25.

Moeller, A. et al., 2016. Social behavior shapes the chimpanzee pan-microbiome. Science Advances, 2 , p.e1500997.

Montoya, J.M.J.M. et al., 2002. Small world patterns in food webs. Journal of theoretical biology, 214(3), pp.405-12.

Moorthy, A.S. et al., 2015. A Spatially Continuous Model of Carbohydrate Digestion and Transport Processes in the Colon. PloS one, 10(12), p.e0145309.

Morris, J. et al., 2012. The Black Queen Hypothesis: Evolution of Dependencies through Adaptive Gene Loss.mBio, 3(2):e00036-12

Mouquet, N. and Loreau, M., 2002. Coexistence in Metacommunities: The Regional Similarity Hypothesis. The American Naturalist, 159(4), pp.420-426.

Murall, C.L., McCann, K.S. and Bauch, C.T., 2012. Food webs in the human body: Linking ecological theory to viral dynamics T. N. Romanuk, ed. PLOS ONE, 7(11), p.e48812.

Murall, C.L., McCann, K.S. and Bauch, C.T., 2014. Revising ecological assumptions about Human Papillomavirus interactions and type replacement. Journal of theoretical biology, 350, pp.98-109.

Murray, J.L. et al., 2014. Mechanisms of synergy in polymicrobial infections. Journal of Microbiology, 52(3), pp.188-199.

Mushegian, A.A. and Ebert, D., 2016. Rethinking "mutualism" in diverse host-symbiont communities. BioEssays, 38(1), pp.100-108.

Naik, S. et al., 2012. Compartmentalized Control of Skin Immunity by Resident Commensals. Science, 337 , pp.1115-1120.

Namba, T., 2015. Multi-faceted approaches toward unravelling complex ecological networks. Population Ecology, pp.3-19.

Naqvi, A. et al., 2010. Network-based modeling of the human gut microbiome. Chemistry and biodiversity, 7(5), pp.1040-1050.

van Nes, E.H. and Scheffer, M., 2005. Implications of Spatial Heterogeneity for Catastrophic Regime Shifts in Ecosystems. Ecology, 86(7), pp.1797-1807.

Newman, M.E.J., 2003. The structure and function of complex networks. Cond-Mat/0303516, 45(2), pp.167-256.

Nobbs, A.H. and Jenkinson, H.F., 2015. Interkingdom networking within the oral microbiome. Microbes and Infection, 17(7), pp.484-492.

Nobel, Y.R. et al., 2015. Metabolic and metagenomic outcomes from early-life pulsed antibiotic treatment. Nature communications, 6(May), p.7486.

van Nood, E. et al., 2013. Duodenal infusion of donor feces for recurrent Clostridium difficile. N Engl J Med, 368(5), pp.407-415.

Norman, J.M. et al., 2015. Disease-specific Alterations in the Enteric Virome in Inflammatory Bowel 
Disease. Cell, 160(3), pp.447-460.

Nowell, P.C., 1976. The clonal evolution of tumor cell populations. Science, 194, pp.23-28.

O'Gorman, E.J. et al., 2010. Interaction strength, food web topology and the relative importance of species in food webs. The Journal of animal ecology, 79(3), pp.682-92.

O'Keefe, S.J.D. et al., 2015. Fat, fibre and cancer risk in African Americans and rural Africans. Nature communications, 6(May 2014), p.6342.

Oh, J. et al., 2013. The altered landscape of the human skin microbiome in patients with primary immunodeficiencies. Genome Research, 23, pp.2103-2114.

Ooi, L.G. and Liong, M.T., 2010. Cholesterol-lowering effects of probiotics and prebiotics: A review of in Vivo and in Vitro Findings. International Journal of Molecular Sciences, 11(6), pp.2499-2522.

Orlandi, J. et al., 2014. First Connectomics Challenge: From Imaging to Connectivity. JMLR: Workshop and Conference Proceedings, 1, pp.1-17.

Ostaff, M.J., Stange, E.F. and Wehkamp, J., 2013. Antimicrobial peptides and gut microbiota in homeostasis and pathology. EMBO Molecular Medicine, 5(10), pp.1465-1483.

De Paepe, M. et al., 2014. Bacteriophages: an underestimated role in human and animal health? Frontiers in cellular and infection microbiology, 4(March), p.39.

De Paepe, M. et al., 2016. Carriage of $\lambda$ Latent Virus Is Costly for Its Bacterial Host due to Frequent Reactivation in Monoxenic Mouse Intestine. PLoS Genetics, 12(2), pp.1-20.

Paine, R.T., 1969. A note on trophic complexity and community stability. The American Naturalist, 103(929), pp.91-93.

Paine, R.T., 1966. Food web complexity and species diversity. American Naturalist, 100, pp.65-75.

Pantel J.H., Bohan D., Calcagno V., David P., Duyck P.-F., Kamenova S., Loeuille N., Mollot G., Romanuk T., Thébault E., Tixier P. \& Massol F. (in press). 14 questions for invasion in ecological networks. Adv. Ecol. Res., 56.

Parfrey, L.W., Walters, W.A. and Knight, R., 2011. Microbial eukaryotes in the human microbiome: Ecology, evolution, and future directions. Frontiers in Microbiology, 2(JULY), pp.1-6.

Pedersen, A.B. and Fenton, A., 2007. Emphasizing the ecology in parasite community ecology. Trends in ecology and evolution, 22(3), pp.133-9.

Pepper, J.W. and Rosenfeld, S., 2012. The emerging medical ecology of the human gut microbiome. Trends in ecology and evolution, 27(7), pp.381-4.

Perelson, A.S., 2002. Modelling viral and immune system dynamics. Nature reviews Immunology, 2(1), pp.28-36.

Perry, S. et al., 2010. Infection with Helicobacter pylori is associated with protection against tuberculosis. PLOS ONE, 5(1).

Petraitis, P.S., 1979. Competitive networks and measures of intransitivity. The American Naturalist, 114(6), pp.921-5.

Petrof, E.O. et al., 2013. Microbial ecosystems therapeutics: A new paradigm in medicine? Beneficial Microbes, 4(1), pp.53-65.

Peura, S. et al., 2015. Resistant microbial cooccurrence patterns inferred by network topology. Applied and Environmental Microbiology, 81(6), pp.2090-2097.

Pilyugin, S.S. and Antia, R., 2000. Modeling immune responses with handling time. Bulletin of mathematical biology, 62(5), pp.869-90.

Pollitt, E.J.G. et al., 2014. Cooperation, quorum sensing, and evolution of virulence in Staphylococcus aureus. Infection and Immunity, 82(3), pp.1045-1051.

Proulx, S.R., Promislow, D.E.L. and Phillips, P.C., 2005. Network thinking in ecology and evolution. Trends in Ecology and Evolution, 20(6 SPEC. ISS.), pp.345-353.

Puillandre, N. et al., 2012. ABGD, Automatic Barcode Gap Discovery for primary species delimitation. Molecular Ecology, 21, pp.1864-1877.

Pybus, V. and Onderdonk, A.B., 1997. Evidence for a commensal, symbiotic relationship between Gardnerella vaginalis and Prevotella bivia involving ammonia: potential significance for bacterial vaginosis. The Journal of Infectious Diseases, 175, pp.406-413.

Rader, B.A. and Guilemin, K., 2013. Insights into the human microbiome from animal models. In D. N. Fredricks, ed. The Human Microbiota: how microbial communities affect health and disease. Wiley Online Library, p. 389.

Ramanan, D. et al., 2016. Helminth infection promotes colonization resistance via type 2 immunity. Science, 352, pp.608-12. 
Rankin, D.J., Rocha, E.P.C. and Brown, S.P., 2011. What traits are carried on mobile genetic elements, and why? Heredity, 106(1), pp.1-10.

Ravel, J. et al., 2013. Daily temporal dynamics of vaginal microbiota before, during and after episodes of bacterial vaginosis. Microbiome, 1(1), p.29.

Ravel, J. et al., 2011. Vaginal microbiome of reproductive-age women. Pnas, 108(Supplement 1), pp.4680-87.

Ray, A. and Jones, C., 2016. Does the donor matter? Future Microbiology, 11(5), pp.611-616.

Read, A.F. and Taylor, L.H., 2001. The ecology of genetically diverse infections. Science, 292(5519), pp.1099-102.

Relman, D.A., 2012. The human microbiome: Ecosystem resilience and health. Nutrition Reviews, 70(SUPPL. 1).

Ribet, D. and Cossart, P., 2015. How bacterial pathogens colonize their hosts and invade deeper tissues. Microbes and Infection, 17(3), pp.173-183.

Ridaura, V.K. et al., 2013. Gut Microbiota from Twins Discordant for Obesity Modulate Metabolism in Mice Gut Microbiota from Twins Metabolism in Mice. Science, 341(September), p.1241214.

Riquet, F.B. et al., 2008. Impact of Exogenous Sequences on the Characteristics of an Epidemic Type 2 Recombinant Vaccine-Derived Poliovirus. Journal of Virology, 82(17), pp.8927-8932.

Ritchie, M.L., 2011. Structure and function of the human microbiome. Dalhousie University.

Robinson, C.J. and Young, V.B., 2010. Antibiotic administration alters the community structure of the gastrointestinal microbiota. Gut microbes, 1(4), pp.279-284.

Rogers, G.B. et al., 2010. Revealing the dynamics of polymicrobial infections: Implications for antibiotic therapy. Trends in Microbiology, 18(8), pp.357-364.

Rogers, S. et al., 2008. Investigating the correspondence between transcriptomic and proteomic expression profiles using coupled cluster models. Bioinformatics, 24(24), pp.2894-2900.

Rohmer, L., Hocquet, D. and Miller, S.I., 2011. Are pathogenic bacteria just looking for food? Metabolism and microbial pathogenesis. Trends in Microbiology, 19(7), pp.341-348.

Rohr, R.P., Saavedra, S. and Bascompte, J., 2014. On the structural stability of mutualistic systems. Science, 345(6195), p.1253497.

Romanuk, T.N. et al., 2009. Predicting invasion success in complex ecological networks. Philosophical transactions of the Royal Society of London. Series B, Biological sciences, 364(1524), pp.1743-54.

Romero, R. et al., 2014. The composition and stability of the vaginal microbiota of normal pregnant women is different from that of non-pregnant women. Microbiome, 2(1), p.4.

de Roode, J.C. et al., 2005. Virulence and competitive ability in genetically diverse malaria infections. Proceedings of the National Academy of Sciences of the United States of America, 102(21), pp.7624-8.

Rooney, N. et al., 2006. Structural asymmetry and the stability of diverse food webs. Nature, 442(July), pp.265-269.

Rooney, N. and McCann, K.S., 2012. Integrating food web diversity, structure and stability. Trends in ecology and evolution, 27(1), pp.40-6.

Rossi-Tamisier, M. et al., 2015. Cautionary tale of using 16S rRNA gene sequence similarity values in identification of human-associated bacterial species. International Journal of Systematic and Evolutionary Microbiology, 65(6), pp.1929-1934.

Sadd, B.M. and Schmid-Hempel, P., 2009. Principles of ecological immunology. Evolutionary Applications, 2(1), pp.113-121.

Saleem, M., 2015. Microbiome Community Ecology: Fundamentals and Applications Briefs., Springer International Publishing.

Sanford, J.A. and Gallo, R.L., 2013. Seminars in Immunology Functions of the skin microbiota in health and disease. Seminars in Immunology, 25(5), pp.370-377.

Santos, S.B. et al., 2009. The use of antibiotics to improve phage detection and enumeration by the double-layer agar technique. BMC microbiology, 9(1), p.148.

Sasaki, A. and Iwasa, Y., 1991. Optimal Growth Switching Schedule of Pathogens within a Host: between Lytic and Latent Cycles. Theoretical Population Biology, 39, pp.201-239.

Sax, D.F. et al., 2007. Ecological and evolutionary insights from species invasions. Trends in Ecology and Evolution, 22(9), pp.465-471.

Scheffer, M. et al., 2001. Catastrophic shifts in ecosystems. Nature, 413(6856), pp.591-6.

Scheffer, M. and Carpenter, S.R., 2003. Catastrophic regime shifts in ecosystems: Linking theory to 
observation. Trends in Ecology and Evolution, 18(12), pp.648-656.

Schreiber, S.J. and Rittenhouse, S., 2004. From simple rules to cycling in community assembly. Oikos, 105(2), pp.349-358.

Schuch, R. and Fischetti, V.A., 2006. Detailed Genomic Analysis of the Wbeta and gamma Phages Infecting Bacillus anthracis: Implications for evolution of environmental fitness and antibiotic resistance. Microbiology, 188(8), pp.3037-3051.

Seabloom, E.W. et al., 2015. The community ecology of pathogens: coinfection, coexistence and community composition. Ecology Letters, 18, p.401-15

Seedorf, H. et al., 2014. Bacteria from diverse habitats colonize and compete in the mouse gut. Cell, 159(2), pp.253-266.

Seekatz, A.M., Aas, J. and Gessert, C.E., 2014. Recovery of the Gut Microbiome following Fecal Microbiota. , 5(3), pp.1-9.

Shafquat, A. et al., 2014. Functional and phylogenetic assembly of microbial communities in the human microbiome. Trends in Microbiology, 22(5), pp.261-266.

Shahinas, D. et al., 2012. Toward an understanding of changes in diversity. mBio, 3(5), pp.e00338-12.

Shankar, V. et al., 2014. Species and genus level resolution analysis of gut microbiota in Clostridium difficile patients following fecal microbiota transplantation. Microbiome, 2(1), p.13.

Shearer, W.T. et al., 2014. Recommendations for live viral and bacterial vaccines in immunodeficient patients and their close contacts. Journal of Allergy and Clinical Immunology, 133(4), pp.961-966.

Sibley, C.D. et al., 2008. Discerning the complexity of community interactions using a Drosophila model of polymicrobial infections. PLoS Pathogens, 4(10).

Simberloff, D., 2009. The Role of Propagule Pressure in Biological Invasions.

Simberloff, D. and Holle, B. Von, 1999. Positive interactions of nonindigenous species: invasional meltdown? Biological Invasions, pp.21-32.

Smith, J., 2001. The social evolution of bacterial pathogenesis. Proceedings of the Royal Society B: Biological Sciences, 268(1462), pp.61-69.

Smith, V.H. and Holt, R.D., 1996. Resource Competition and within-Host Disease Dynamics. Trends in Ecology and Evolution, 11(9), pp.386-389.

Smith-Ramesh, L.M., Moore, A.C. and Schmitz, O.J., 2016. Global synthesis suggests that food web connectance correlates to invasion resistance. Global Change Biology, pp.1-9.

Sofonea, M.T., Alizon, S. and Michalakis, Y., 2015. From within-host interactions to epidemiological competition: a general model for multiple infections. Philosophical Transactions of the Royal Society B: Biological Sciences, 370(1675), p.20140303.

Solé, R. V and Montoya, J.M., 2001. Complexity and fragility in ecological networks. Proceedings of the Royal Society B: Biological Sciences, 268(1480), pp.2039-45.

Sommer, S., 2005. The importance of immune gene variability (MHC) in evolutionary ecology and conservation. Frontiers in zoology, 2, p.16.

Song, H.-S. et al., 2014. Mathematical Modeling of Microbial Community Dynamics: A Methodological Review. Processes, 2(4), pp.711-752.

Stecher, B. et al., 2010. Like will to like: Abundances of closely related species can predict susceptibility to intestinal colonization by pathogenic and commensal bacteria. PLoS Pathogens, 6(1).

Stecher, B. and Hardt, W.-D., 2011. Mechanisms controlling pathogen colonization of the gut. Current opinion in microbiology, 14(1), pp.82-91.

Stecher, B., Maier, L. and Hardt, W.-D., 2013. "Blooming" in the gut: how dysbiosis might contribute to pathogen evolution. Nature reviews. Microbiology, 11(4), pp.277-84.

Stein, R.R. et al., 2013. Ecological modeling from time-series inference: insight into dynamics and stability of intestinal microbiota. PLoS computational biology, 9(12), p.e1003388.

Stressmann, F.A. et al., 2012. Long-term cultivation-independent microbial diversity analysis demonstrates that bacterial communities infecting the adult cystic fibrosis lung show stability and resilience. Thorax, 67(10), pp.867-73.

Suez, J. et al., 2014. Artificial sweeteners induce glucose intolerance by altering the gut microbiota. Nature, 514(7521), pp.181-186.

Sugihara, G. et al., 2012. Detecting causality in complex ecosystems. Science, 338, pp.496-500.

Sulakvelidze, A., 2011. The challenges of bacteriophage therapy. Industrial Pharmacy, 45(31), pp.14-18.

Swidsinski, A. et al., 2005. Adherent biofilms in bacterial vaginosis. Obstetrics and gynecology, 106(5 Pt 1), pp.1013-1023. 
Swidsinski, A. et al., 2014. Infection through structured polymicrobial Gardnerella biofilms (StPM-GB). Histology and Histopathology, 29(5), pp.567-587.

Tasiemski, A. et al., 2015. Reciprocal immune benefit based on complementary production of antibiotics by the leech Hirudo verbana and its gut symbiont Aeromonas veronii. Scientific Reports, 5, p.17498.

Tatusov, R.L. et al., 2003. The COG database: an updated version includes eukaryotes. BMC bioinformatics, 4, p.41.

Taur, Y. et al., 2012. Intestinal domination and the risk of bacteremia in patients undergoing allogeneic hematopoietic stem cell transplantation. Clinical Infectious Diseases, 55(7), pp.905-914.

Theoharides, K. a \& Dukes, J.S., 2007. Plant invasion across space and time: factors affecting nonindigenous species success during four stage of invasion. New Phytologist, 176(2), pp.256-273.

Tillmann, H.L. \& Al., E., 2001. Infection with GB virus C and reduced mortality among HIV-infected patients. New England Journal of Medicine, 345(10), pp.715-724.

Tilman, D., 1999. The ecological consequences of changes in biodiversity: a search for general principles. Ecology, 80(5), pp.1455-1474.

Toh, M.C. et al., 2013. Colonizing the embryonic zebrafish gut with anaerobic bacteria derived from the human gastrointestinal tract. Zebrafish, 10(2), pp.194-198.

Torres-Barceló, C. et al., 2016. Long-term effects of single and combined introductions of antibiotics and bacteriophages on populations of Pseudomonas aeruginosa. Evolutionary Applications, 9(4), pp.583-595.

Torres-Barcelo, C. and Hochberg, M.E., 2016. Evolutionary Rationale for Phages as Complements of Antibiotics. Trends in Microbiology, 24(4), pp.249-256.

Tremblay, J. et al., 2015. Primer and platform effects on 16S rRNA tag sequencing. Frontiers in Microbiology, 6(AUG), pp.1-15.

Trosvik, P. and de Muinck, E.J., 2015. Ecology of bacteria in the human gastrointestinal tractidentification of keystone and foundation taxa. Microbiome, 3(1), p.44.

Turovskiy, Y. et al., 2011. The etiology of Bacterial Vaginosis. Journal of Applied Microbiology, 110(5), pp.1105-1128.

Underhill, D.M. and Iliev, I.D., 2014. The mycobiota: interactions between commensal fungi and the host immune system. Nature reviews. Immunology, 14(6), pp.405-16.

Vacher, C. et al., 2016. Learning Ecological Networks from Next-Generation Sequencing Data. Advances in Ecological Research, 54, pp.1-39.

Vale, P.F. et al., 2016. Beyond killing: Can we find ways to manage infection? Evolution, Medicine, and Public Health, (1), pp.148-157.

Venema, K. and van den Abbeele, P., 2013. Experimental models of the gut microbiome. Best practice and research. Clinical gastroenterology, 27(1), pp.115-26.

Vrieze, A. et al., 2012. Transfer of intestinal microbiota from lean donors increases insulin sensitivity in individuals with metabolic syndrome. Gastroenterology, 143(4), p.913-916.e7.

Wegner, K.M. et al., 2003. Parasite selection for immunogenetic optimality. Science, 301, p.1343.

Wexler, A.G. et al., 2016. Human symbionts inject and neutralize antibacterial toxins to persist in the gut. Proceedings of the National Academy of Sciences, 113(13), pp.3639-3644.

Winternitz, J.C. and Abbate, J.L., 2015. Examining the evidence for major histocompatibility complexdependent mate selection in humans and nonhuman primates. Research and Reports in Biology, (6), pp.73-88.

Wissenbach, D.K. et al., 2016. Optimization of Metabolic profiling and characterization of defined in vitro gut microbial ecosystems. International Journal of Medical Microbiology.

Wodarz, D., 2006. Ecological and evolutionary principles in immunology. Ecology Letters, 9(6), pp.694705.

Wolfe, B.E. and Dutton, R.J., 2015. Fermented foods as experimentally tractable microbial ecosystems. Cell, 161(1), pp.49-55.

Wylie, K.M., Weinstock, G.M. and Storch, G. A, 2013. Virome genomics: a tool for defining the human virome. Current opinion in microbiology, 16(4), pp.479-84.

Xiang, J. et al., 2001. Effect of coinfection with GB virus $C$ on survival among patients with HIV infection. The New England journal of medicine, 345(10), pp.707-714.

Yager, E.J. et al., 2009. Y-Herpesvirus-Induced Protection Against Bacterial Infection Is Transient. Viral Immunology, 22(1), pp.67-71.

Ye, H. and Sugihara, G., 2016. Information leverage in interconnected ecosystems: Overcoming the 
curse of dimensionality. Science, 353(6302), pp.922-925.

Yen, S. et al., 2015. Metabolomic analysis of human fecal microbiota: A comparison of feces-derived communities and defined mixed communities. Journal of Proteome Research, 14(3), pp.1472-1482.

Yezli, S. and Otter, J.A., 2011. Minimum Infective Dose of the Major Human Respiratory and Enteric Viruses Transmitted Through Food and the Environment. Food and Environmental Virology, 3(1), pp.1-30.

Zaiss, M.M. and Harris, N.L., 2016. Interactions between the intestinal microbiome and helminth parasites. Parasite Immunology, 38(1), pp.5-11.

Zhang, C. et al., 2016. Ecological robustness of the gut microbiota in response to ingestion of transient food-borne microbes. The ISME journal, pp.1-11.

Zhou, J. et al., 2010. Functional molecular ecological networks. MBio, 1(4), pp.e00169-10.

Zhou, Y. et al., 2013. Biogeography of the ecosystems of the healthy human body. Genome biology, 14(1), p.R1.

Zhou, Y. et al., 2014. Exploration of bacterial community classes in major human habitats. Genome biology, 15(5), p.R66. 